ESAIM: COCV 27 (2021) 32

https://doi.org/10.1051/cocv/2021024
ESAIM: Control, Optimisation and Calculus of Variations

www.esaim-cocv.org

\title{
EXTREMALS FOR A SERIES OF SUB-FINSLER PROBLEMS WITH 2-DIMENSIONAL CONTROL VIA CONVEX TRIGONOMETRY ${ }^{*, * *, * * *, * * * *}$
}

\author{
A.A. Ardentov ${ }^{1, * * * *}$, L.V. Lokutsievskiy ${ }^{2}$ and Yu.L. SAChkov ${ }^{3}$
}

\begin{abstract}
We consider a series of optimal control problems with 2-dimensional control lying in an arbitrary convex compact set $\Omega$. The considered problems are well studied for the case when $\Omega$ is a unit disc, but barely studied for arbitrary $\Omega$. We derive extremals to these problems in general case by using machinery of convex trigonometry, which allows us to do this identically and independently on the shape of $\Omega$. The paper describes geodesics in (i) the Finsler problem on the Lobachevsky hyperbolic plane; (ii) left-invariant sub-Finsler problems on all unimodular 3D Lie groups $(\mathrm{SU}(2), \mathrm{SL}(2), \mathrm{SE}(2)$, $\mathrm{SH}(2)$ ); (iii) the problem of rolling ball on a plane with distance function given by $\Omega$; (iv) a series of "yacht problems" generalizing Euler's elastic problem, Markov-Dubins problem, Reeds-Shepp problem and a new sub-Riemannian problem on $\mathrm{SE}(2)$; and (v) the plane dynamic motion problem.
\end{abstract}

Mathematics Subject Classification. 49Q99.

Received April 26, 2020. Accepted February 25, 2021.

\section{INTRODUCTION}

In this paper, we obtain extremals in a series of optimal control problems with two-dimensional control lying in a compact convex set $\Omega \subset \mathbb{R}^{2}, 0 \in$ int $\Omega$. Usually Pontryagin maximum principle dictates an optimal control to move along the boundary $\partial \Omega$. In the case, when $\partial \Omega$ is the unit circle, this motion can be conveniently described by the trigonometric functions cos and sin. This allows to find explicit formulas for extremals in a lot of optimal control problems with $\Omega$ being the unit disc, which in fact is very useful for any deep investigation of a problem. In the case, when $\Omega$ has an arbitrary form, finding formulas for extremals is much more difficult task

* Section 6 was written by A.A. Ardentov. Sections 1-3, 5 , 7 were written by L.V. Lokutsievskiy. Section 4 was written by Yu.L. Sachkov. All results in this paper are products of authors collaborative work.

** The work of A.A. Ardentov is supported by the Russian Science Foundation under grant 17-11-01387-p and performed in Ailamazyan Program Systems Institute of Russian Academy of Sciences.

*** The work of L.V. Lokutsievskiy is supported by the Russian Science Foundation under grant 20-11-20169 and performed in Steklov Mathematical Institute of Russian Academy of Sciences.

**** The work of Yu.L. Sachkov is supported by the Russian Foundation for Basic Research, project number 19-31-51023.

Keywords and phrases: Sub-Finsler geometry, convex trigonometry, optimal control problem, Lobachevsky hyperbolic plane, unimodular 3D Lie group, yacht problems, rolling ball.

1 Ailamazyan Program Systems Institute, Russian Academy of Sciences, Pereslavl-Zalessky, Russia.

2 Steklov Mathematical Institute of Russian Academy of Sciences, Moscow, Russia.

3 Sirius University of Science and Technology, Sochi, Russia.

***** Corresponding author: aaa@pereslavl.ru 
also because extremals depend on the structure of $\Omega$. Hence, a lot of important problems (which are very well studied when $\Omega$ is the unit disc) are barely investigated or even not investigated at all if $\Omega$ has some different shape. It seems to us, it happens because of lack of a convenient way to describe motions of the optimal control along the boundary of an arbitrary flat convex set.

In the present paper, we use new functions $\cos _{\Omega}$ and $\sin _{\Omega}$ (which were introduced in [31]) to describe this motion. These new functions inherit a lot of convenient properties from the classical functions cos and sin (we usually use the term "convex trigonometry" for this collection of properties). On the one hand, convex trigonometry allows us to derive short formulas for extremals in terms of $\cos _{\Omega}$ and $\sin _{\Omega}$ in a series of optimal control problems. On the other hand, the functions $\cos _{\Omega}$ and $\sin _{\Omega}$ can be expressed by classic functions for a lot of concrete sets $\Omega$ (see Sect. 2.3 for the detailed list of the sets). For example, if $\Omega=\left\{|x|^{p}+|y|^{p} \leq 1\right\}$, $1 \leq p \leq \infty$, the functions $\cos _{\Omega}$ and $\sin _{\Omega}$ coincide with well known Shelupsky's functions and can be expressed in terms of Euler beta function or hypergeometric function ${ }_{2} F_{1}$.

So the following new optimal control problems are investigated in the present paper.

- In Section 3, we find Finsler geodesics on the Lobachevsky hyperbolic plane. This problem is equivalent to a left-invariant Finsler problem on the group Aff $(\mathbb{R})$ of proper affine transformations of the real line $\mathbb{R}$. For centrally symmetrical sets $\Omega$, this problem was investigated in [27]. In the present paper, we find all geodesics for all possible sets $\Omega$. The result is obtained by convex trigonometry, but the final answer does not contain the functions $\cos _{\Omega}$ and $\sin _{\Omega}$ at all (see Thm. 3.1).

- Left-invariant sub-Finsler problems on 3D unimodular Lie groups considered in Section 5 are a natural generalization of similar sub-Riemannian problems (recall that a problem is sub-Riemannian if $\Omega$ is an ellipse centered at the origin). Sub-Riemannian geodesics on all these groups can be parameterized by elliptic functions, see Section 18 of [3]. Moreover, in some of these problems, an optimal synthesis is known (see [45] for the Heisenberg group $\mathrm{H}_{3},[11,13]$ for some $\mathrm{SO}(3)$ and $\mathrm{SL}(2)$ cases, [34, 41, 42] for $\mathrm{SE}(2)$, and [18-20] for $\mathrm{SH}(2))$. Left invariant sub-Riemannian and sub-Finsler problems are of great importance for control theory and mathematics in general $[3,8,9,16,35]$, and arise in a lot of practical applications, see e.g. [14, 15, 29, 43]. Sub-Finsler problems on SE(2) take important role for building control models of wheeled robots, e.g. famous Reeds-Shepp car [39] corresponds to $l_{\infty}$ case (see Sect. 5.8, Case 4), shortest paths for differential-drive mobile robot with the restriction on wheel-rotation speed $[7,21]$ are equavalent to sub-Finsler minimizers on $\mathrm{SE}(2)$ with $l_{1}$ norm (see Sect. 5.9, Case 4). The generalization of such models on polygonal and strictly convex smooth boundaries is explored in [6]. Nonetheless, for sub-Finsler problems (when $\Omega$ is arbitrary), only geodesics on the Heisenberg group $\mathrm{H}_{3}$ are known (see [10, 17]). In the present paper, for arbitrary $\Omega$, we find solutions to the vertical subsystem of the Pontryagin maximum principle in terms of convex trigonometry (see Sect. 5). For example, this allows us to fully describe the phase portrait of the system and give a full description of singular extremals and their types in the $\ell_{p}$ case (see Sects. 5.8-5.10).

- In Section 6, we consider a sphere rolling on a plane without slipping or twisting. The problem is to find a rolling of the sphere from one given position to another such that the center draws a shortest possible path and the sphere rotates in a given way. In the classical case, when the path length is measured by the Euclidean distance, the problem is well studied (see [29, 30]). For example, it is known that optimal motions of the center coincide with Euler elasticae. In Section 6, we investigate an unsolved problem when the path length of the sphere center is measured by an arbitrary sub-Finsler metric on the plane. We find solutions to the vertical subsystem of the Pontryagin maximum principle in terms of convex trigonometry. In particular, this allows us to describe behavior of optimal sphere motions when the sub-Finsler metric is piecewise linear (see Thm. 6.2).

- In Section 7, we define a series of optimal control problems generalizing four classic problems (Euler elasticae, Dubins car, Reeds-Shepp car) and a new sub-Riemannian problem on $\mathrm{SE}(2)$, which express control models for a car-like robot. All the listed problems were investigated in literature (see [24] for Euler elasticae, [23] for Dubins car, [39] for Reeds-Shepp car, [34, 41, 42] for the sub-Riemannian problem on $\mathrm{SE}(2))$. We use arbitrary convex set $\Omega$ of unit velocities on the plane to generalize those models as yacht 
models moving in a sea (or a car moving on a non-horizontal plane). ${ }^{1}$ For each case we investigate the phase portrait for the vertical subsystem of the Hamiltonian system and study possible extremals for arbitrary $\Omega$. A special type of extremals appears for not strictly convex $\Omega$ with edges. For the Markov-Dubins generalization, we describe all extremal controls explicitly in Theorems 7.5, 7.6. For the Reeds-Shepp generalization, we find all patterns for control switchings. For other problems, we use the theory developed in Section 4 to classify all types of extremal controls.

- In Section 8, we consider a system with drift that describes a controlled motion of a massive point on a plane, where the maximum admissible acceleration depends on the chosen direction. This problem generalizes the classic one-dimensional Pontryagin train control problem to the dimension 2.

The paper has the following structure. In Section 2, we shortly introduce convex trigonometry, a list of sets $\Omega$ for which the functions $\cos _{\Omega}$ and $\sin _{\Omega}$ are computed explicitly, and some new results on smoothness of these functions. Section 4 contains important results on second order ODEs expressed by functions of convex trigonometry. These results are used in all consequent sections. Sections $3,5-8$ contain investigation of the optimal control problems described above.

\section{Convex trigonometry}

Let $\Omega \subset \mathbb{R}^{2}$ be a convex compact set and let $0 \in \operatorname{int} \Omega$. In this section, we give a brief introduction to convex trigonometry, which was introduced in [31]. In the present paper, we use convex trigonometry in all the optimal control problems mentioned in the introduction.

\subsection{Notation and definitions}

Denote by $\mathbb{S}$ the area of the set $\Omega$.

Definition 2.1. Let $\theta \in \mathbb{R}$ denote a generalized angle. If $0 \leq \theta<2 \mathbb{S}$, then we choose a point $P_{\theta}$ on the boundary of $\Omega$ such that the area of the sector of $\Omega$ between the rays $O x$ and $O P_{\theta}$ is $\frac{1}{2} \theta$. By definition $\cos _{\Omega} \theta$ and $\sin _{\Omega} \theta$ are the coordinates of $P_{\theta}$. If the generalized angle $\theta$ does not belong to the interval $[0 ; 2 \mathbb{S})$, then we define the functions $\cos _{\Omega}$ and $\sin _{\Omega}$ as periodic with the period $2 \mathbb{S}$; i.e., for $k \in \mathbb{Z}$ such that $\theta+2 \mathbb{S} k \in[0 ; 2 \mathbb{S})$ we put

$$
\cos _{\Omega} \theta=\cos _{\Omega}(\theta+2 \mathbb{S} k) ; \quad \sin _{\Omega} \theta=\sin _{\Omega}(\theta+2 \mathbb{S} k) ; \quad P_{\theta}=P_{\theta+2 \mathbb{S} k} .
$$

If $\Omega$ is the unit disc, $\Omega=\left\{x^{2}+y^{2} \leq 1\right\}$, then the above definition produces the classic trigonometric functions $\cos$ and sin.

We will use the polar set $\Omega^{\circ}$ together with the set $\Omega$ :

$$
\Omega^{\circ}=\left\{(p, q) \in \mathbb{R}^{2 *}: p x+q y \leq 1 \text { for all }(x, y) \in \Omega\right\} \subset \mathbb{R}^{2 *} .
$$

The polar set $\Omega^{\circ}$ is (always) a convex and compact (as $0 \in \operatorname{int} \Omega$ ) set and $0 \in \operatorname{int} \Omega^{\circ}$ (as $\Omega$ is bounded). To avoid confusion we will assume that the set $\Omega$ lies in the plane with coordinates $(x, y)$ and the polar set $\Omega^{\circ}$ lies in the plane with coordinates $(p, q)$.

Note that $\Omega^{\circ \circ}=\Omega$ by the bipolar theorem (see [40], Thm. 14.5). We can apply the above definition of the generalized trigonometric functions to the polar set $\Omega^{\circ}$ and an arbitrary angle $\psi \in \mathbb{R}$ to construct $\cos _{\Omega^{\circ}} \psi$ and $\sin _{\Omega^{\circ}} \psi$, which are the coordinates of the appropriate point $Q_{\psi} \in \partial \Omega^{\circ}$.

Definition 2.2. We say that angles $\theta \in \mathbb{R}$ and $\theta^{\circ} \in \mathbb{R}$ correspond to each other and write $\theta \stackrel{\Omega}{\longleftrightarrow} \theta^{\circ}$ if the supporting half-plane of $\Omega$ at $P_{\theta}$ is determined by the (co)vector $Q_{\theta^{\circ}}$. When no confusing ensues we omit the symbol $\Omega$ over the arrow and write $\theta \leftrightarrow \theta^{\circ}$.

\footnotetext{
${ }^{1}$ Yachts problems are very popular applications of control theory nowadays (see $[22,25,36,38,47]$ ). Usually authors consider these problems for some specific set $\Omega$ of a given shape. Our approach allows us to investigate these problems simultaneously for all possible sets $\Omega$.
} 


\subsection{Main properties of convex trigonometry}

Properties of the classic functions $(\cos , \sin )$ are inherited by two pairs of functions $\left(\cos _{\Omega}, \sin _{\Omega}\right)$ and $\left(\cos \Omega^{\circ}, \sin _{\Omega^{\circ}}\right)$ for the sets $\Omega$ and $\Omega^{\circ}$. All the listed below properties are proved in Section 2 of [31].

I. Generalized Pythagorean identity

$$
\theta \stackrel{\Omega}{\longleftrightarrow} \theta^{\circ} \Longleftrightarrow \theta^{\circ} \stackrel{\Omega^{\circ}}{\longleftrightarrow} \theta \Longleftrightarrow \cos _{\Omega} \theta \cos _{\Omega^{\circ}} \theta^{\circ}+\sin _{\Omega} \theta \sin _{\Omega^{\circ}} \theta^{\circ}=1
$$

II. Differentiation formulae. The functions $\cos _{\Omega}$ and $\sin _{\Omega}$ are Lipschitz continuous, and for a.e. $\theta$,

$$
\cos _{\Omega}^{\prime} \theta=-\sin _{\Omega^{\circ}} \theta^{\circ} \quad \text { and } \quad \sin _{\Omega}^{\prime} \theta=\cos _{\Omega^{\circ}} \theta^{\circ}
$$

where $\theta^{\circ} \leftrightarrow \theta$ (in this case, there exists a unique $\theta^{\circ} \leftrightarrow \theta$ ). At points $\theta$ of non-differentiability, the correspondence $\theta^{\circ} \leftrightarrow \theta$ is not bijective, but both functions $\cos _{\Omega}$ and $\sin _{\Omega}$ have right and left derivatives, and the interval between them is given by the right-hand sides in (2.2) for all $\theta^{\circ} \leftrightarrow \theta$.

III. Quasiperiodicity of the dependence $\theta^{\circ}(\theta)$. Let $\mathbb{S}^{\circ}$ denote the area of $\Omega^{\circ}$. Obviously, the angle $\theta^{\circ}$ corresponding to $\theta$ is defined up to $2 \mathbb{S}^{\circ}$, but we are able to choose a monotone function $\theta^{\circ}(\theta) \leftrightarrow \theta$. Then

$$
\theta^{\circ}(\theta+2 \mathbb{S} k)=\theta^{\circ}(\theta)+2 \mathbb{S}^{\circ} k \quad \text { for } \quad k \in \mathbb{Z} .
$$

IV. Polar change of coordinates. Put $x=r \cos _{\Omega} \theta$ and $y=r \sin _{\Omega} \theta$. Then Jacobi matrix is

$$
J=\left(\begin{array}{cc}
x_{r}^{\prime} & x_{\theta}^{\prime} \\
y_{r}^{\prime} & y_{\theta}^{\prime}
\end{array}\right)=\left(\begin{array}{cc}
\cos _{\Omega} \theta & -r \sin _{\Omega^{\circ}} \theta^{\circ} \\
\sin _{\Omega} \theta & r \cos _{\Omega^{\circ}} \theta^{\circ}
\end{array}\right)
$$

where $\theta^{\circ} \leftrightarrow \theta$. Moreover, $\operatorname{det} J=r$ by the generalized Pythagorean identity.

V. Inverse polar change of coordinates. Let $(x(t), y(t))$ be an absolutely continuous curve that does not pass through the origin, and $x=r \cos _{\Omega} \theta$ and $y=r \sin _{\Omega} \theta$ as above. Then

$$
r=s_{\Omega^{\circ}}(x, y) \quad \text { and } \quad \dot{\theta}=\frac{x \dot{y}-\dot{x} y}{r^{2}} .
$$

The first equation holds for all $t$, and the second one holds for a.e. $t$.

\subsection{List of explicitly computed functions $\cos _{\Omega}$ and $\sin _{\Omega}$.}

In this subsection, we enumerate all known cases of sets $\Omega$ for which it is possible to compute functions of convex trigonometry.

1. Suppose $\Omega$ is a (convex) polyhedron. In this case, the functions $\cos _{\Omega}$ and $\sin _{\Omega}$ are piecewise linear. They have corners when the point $P_{\theta}$ passes through a vertex of $\Omega$. Explicit formulas for $\cos _{\Omega}$ and $\sin _{\Omega}$ are given in Section 3 of [31] in terms of coordinates of vertices.

2. Suppose $\Omega=\left\{|x|^{p}+|y|^{p} \leq 1\right\}$ for some $1 \leq p \leq \infty$. If $p=1$ or $p=\infty$, then $\Omega$ is a square and the functions $\cos _{\Omega}$ and $\sin _{\Omega}$ are computed as above. If $1<p<\infty$, then $\cos _{\Omega}$ and $\sin _{\Omega}$ coincide with Shelupcky's generalized trigonometry functions (see [32], Sect. 4). Shelupsky's functions form a 1-parametric family (determined by the parameter $p$ ) of pair of functions $\left(\cos _{p}, \sin _{p}\right)$, whereas the convex trigonometry functions introduced in [31] form an infinite-dimensional family determined by the set $\Omega$. In other words, 
Shelupsky's functions are a special case of convex trigonometry. Shelupsky's function are very convenient for eigenvalue problems for $p$-Laplacians (see $[44,46]$ for details). In this case, the functions $\cos _{\Omega}$ and $\sin _{\Omega}$ can be expressed in terms of the inverse Euler beta function (see [32], Thm. 5).

3. Suppose the boundary of $\Omega$ is defined parametrically $\partial \Omega=\left\{(x(s), y(s)) \in \mathbb{R}^{2} \mid s \in \mathbb{R}\right\}$. Then, for any $s$, there is defined a generalized angle $\theta(s)$ such that $P_{\theta(s)}=(x(s), y(s))$. Obviously,

$$
\cos _{\Omega} \theta(s)=x(s) \quad \text { and } \quad \sin _{\Omega} \theta(s)=y(s) .
$$

Let us compute the function $\theta(s)$ and its inverse $s(\theta)$. Using the inverse polar change of coordinates, we get

$$
\theta_{s}^{\prime}=x y_{s}^{\prime}-x_{s}^{\prime} y \quad \Leftrightarrow \quad \theta(s)=\int\left(x(s) y_{s}^{\prime}(s)-x_{s}^{\prime}(s) y(s)\right) \mathrm{d} s,
$$

since $r(s)=s_{\Omega^{\circ}}(x(s), y(s))=1$. For some cases this integral can be taken explicitly, but it is not possible to do it in general case. Nonetheless, we can often avoid explicit integration here! The main reason is the following: any of the above mentioned optimal control problems can be reduced to an ODE of the form $\dot{\theta}=f\left(\cos _{\Omega} \theta, \sin _{\Omega} \theta\right)$. From $(2.3)$ we have the following ODE:

$$
\dot{s}=\dot{\theta} / \theta_{s}^{\prime}=\frac{f(x(s), y(s))}{x(s) y_{s}^{\prime}(s)-x_{s}^{\prime}(s) y(s)} \quad \Leftrightarrow \quad t=\int \frac{x(s) y_{s}^{\prime}(s)-x_{s}^{\prime}(s) y(s)}{f(x(s), y(s))} \mathrm{d} s .
$$

The last integral does not contain new functions $\cos _{\Omega}$ and $\sin _{\Omega}$, and its computation is reduced to classic calculus.

4. Suppose $\Omega$ is an ellipse. In this case, its boundary is given by $x(s)=a \cos s+x_{0}$ and $y(s)=b \sin s+y_{0}$ (where $\frac{x_{0}^{2}}{a^{2}}+\frac{y_{0}^{2}}{b^{2}}<1$, since $0 \in \operatorname{int} \Omega$ ). Using the previous item we obtain

$$
\theta=a b s-y_{0} a \cos s+x_{0} b \sin s+\text { const. }
$$

We can not find $s(\theta)$ from this equation explicitly. But the function $f(x, y)$ is the square root of a linear or quadratic polynomial in all optimal control problems considered in the paper. Hence, the extremals equation $\dot{\theta}=f\left(\cos _{\Omega} \theta, \sin _{\Omega} \theta\right)$ in these problems takes the form

$$
\dot{s}=\frac{f\left(a \cos s+x_{0}, b \sin s+y_{0}\right)}{a b-y_{0} a \sin s+x_{0} b \cos s} \Leftrightarrow t=\int \frac{a b-y_{0} a \sin s+x_{0} b \cos s}{f\left(a \cos s+x_{0}, b \sin s+y_{0}\right)} \mathrm{d} t,
$$

which can be expressed in elliptic functions.

5. Suppose that the Minkowski function $\mu_{\Omega}=s_{\Omega^{\circ}}$ is known: $\Omega=\left\{(x, y): s_{\Omega^{\circ}}(x, y) \leq 1\right\}$. Let $\phi$ denote a classic angle. Let us parametrize the boundary $\partial \Omega$ by $\phi$, i.e.,

$$
x(\phi)=\cos _{\Omega} \theta(\phi)=\frac{\cos \phi}{s_{\Omega^{\circ}}(\cos \phi, \sin \phi)} \quad \text { and } \quad y(\phi)=\cos _{\Omega} \theta(\phi)=\frac{\sin \phi}{s_{\Omega^{\circ}}(\cos \phi, \sin \phi)} .
$$

Put $r(\phi)=s_{\Omega^{\circ}}(\cos \phi, \sin \phi)$ for short. Then $x+\mathbf{i} y=\frac{1}{r} e^{\mathbf{i} \phi}$. Thus, $\theta^{\prime}=x y^{\prime}-x^{\prime} y$ by the inverse polar change of coordinates. So

$$
\theta^{\prime}=\Re\left((x+\mathbf{i} y) \mathbf{i}\left(x^{\prime}-\mathbf{i} y^{\prime}\right)\right)=\frac{1}{r} \Re\left(\mathbf{i} e^{\mathbf{i} \phi}\left(\frac{1}{r} e^{-\mathbf{i} \phi}\right)^{\prime}\right)=\frac{1}{r} \Re\left(\mathbf{i} e^{\mathbf{i} \phi}\left(-\frac{\mathbf{i}}{r} e^{-\mathbf{i} \phi}-\frac{r^{\prime}}{r^{2}} e^{-\mathbf{i} \phi}\right)\right) .
$$


Hence,

$$
\theta^{\prime}=r^{-2} \Leftrightarrow \theta(\phi)=\int \frac{\mathrm{d} \phi}{r^{2}(\phi)}
$$

The values $\cos _{\Omega} \theta$ and $\sin _{\Omega} \theta$ are equal to $r^{-1} \cos \phi$ and $r^{-1} \sin \phi$ correspondingly. The functions $\cos _{\Omega^{\circ}}$, $\sin _{\Omega^{\circ}}$ and the corresponding angle $\theta^{\circ} \leftrightarrow \theta$ can be found explicitly using differentiation formulae:

$$
\begin{aligned}
& \cos _{\Omega^{\circ}} \theta^{\circ}=\left(\sin _{\Omega} \theta\right)_{\theta}^{\prime}=y_{\phi}^{\prime} / \theta_{\phi}^{\prime}=r^{2} y_{\phi}^{\prime}, \quad \Rightarrow \quad \cos _{\Omega^{\circ}} \theta^{\circ}=r \cos \phi-r^{\prime} \sin \phi ; \\
& \sin _{\Omega^{\circ}} \theta^{\circ}=-\left(\cos _{\Omega} \theta\right)_{\theta}^{\prime}=-x_{\phi}^{\prime} / \theta_{\phi}^{\prime}=-r^{2} x_{\phi}^{\prime} \quad \Rightarrow \quad \sin _{\Omega^{\circ}} \theta^{\circ}=r \sin \phi+r^{\prime} \cos \phi .
\end{aligned}
$$

So, the curve $p+\mathbf{i} q=\left(r+\mathbf{i} r^{\prime}\right) e^{\mathbf{i} \phi}$ describes the boundary of the polar set $\Omega^{\circ}$. Similarly,

$$
\theta^{\circ \prime}=\Re\left(\left(r+\mathbf{i} r^{\prime}\right) e^{\mathbf{i} \phi} \mathbf{i}\left(\left(r-\mathbf{i} r^{\prime}\right) e^{-\mathbf{i} \phi}\right)^{\prime}\right)=\Re\left(\left(r+\mathbf{i} r^{\prime}\right) e^{\mathbf{i} \phi} \mathbf{i} e^{-\mathbf{i} \phi}\left(-\mathbf{i}\left(r+r^{\prime \prime}\right)\right)\right.
$$

Hence,

$$
\theta^{\circ \prime}=r^{2}+r r^{\prime \prime} \quad \Leftrightarrow \quad \theta^{\circ}(\phi)=\int\left(r^{2}(\phi)+r(\phi) r^{\prime \prime}(\phi)\right) \mathrm{d} \phi .
$$

Remark 2.3. Formulae for extremals in the present paper are usually obtained in terms of convex trigonometry. According to item 2.3, if the boundary of $\Omega$ is given parametrically, then all these formulae can be easily rewritten to ones that does not use convex trigonometry at all.

Remark 2.4. If the boundary of $\Omega$ consists of finite number of parts of boundaries of sets $\Omega_{1}, \ldots, \Omega_{N}$, then graphs of functions $\cos _{\Omega}$ and $\sin _{\Omega}$ are glued of corresponding parts of graphs of functions $\cos _{\Omega_{k}}$, $\sin _{\Omega_{k}}$, $1 \leq k \leq \Omega_{N}$. For example, a cut disc is considered in detail in Section 7

\subsection{Smoothness of the functions $\cos \Omega$ and $\sin \Omega$}

Using the formulae of convex trigonometry obtained in the previous subsection, we are able to monitor smoothness of the convex trigonometric functions.

Proposition 2.5. The following statements are equivalent:

(i) the boundary of $\Omega$ is a regular $C^{k}$-curve;

(ii) $\cos _{\Omega}$ and $\sin _{\Omega}$ are $C^{k}$ functions;

(iii) $s_{\Omega^{\circ}}$ is $C^{k}$ outside the origin.

Here $k \geq 1$ is integer, $k=\infty$, or $k=\omega$.

Proof. $(i) \Rightarrow(i i)$. Let the boundary $\partial \Omega$ be given as $(x(s), y(s))$, where $x, y \in C^{k}$, and ${x_{s}^{\prime}}^{2}+y_{s}^{\prime 2} \neq 0$. Then $\theta_{s}^{\prime}(s) \in C^{k-1}$ by (2.4). So $\theta(s) \in C^{k}$. Moreover, $\theta_{s}^{\prime} \neq 0$, since $x_{s}^{\prime 2}+y_{s}^{\prime 2} \neq 0$ and $0 \in \operatorname{int} \Omega$. So the inverse function $s(\theta)$ has the same smoothness, $s(\theta) \in C^{k}$ by the inverse function theorem. Thus $\cos _{\Omega} \theta=x(s(\theta))$ and $\sin _{\Omega}(\theta)=y(s(\theta))$ are $C^{k}$.

$($ ii $) \Rightarrow(i)$. The functions $\cos _{\Omega} \theta$ and $\sin _{\Omega} \theta$ give $C^{k}$ parametrization of the boundary $\partial \Omega$, and $\cos _{\Omega}^{\prime 2} \theta+$ $\sin _{\Omega}^{\prime 2} \theta=\cos _{\Omega^{\circ}}^{2} \theta^{\circ}+\sin _{\Omega^{\circ}}^{2} \theta^{\circ} \neq 0$, since $0 \in \operatorname{int} \Omega^{\circ}$.

(iii) $\Rightarrow\left(\right.$ i). Consider the support function $s_{\Omega^{\circ}}$ in the classic polar coordinates $x=\rho \cos \phi, y=\rho \sin \phi$. Since $s_{\Omega^{\circ}}$ is positively homogeneous, we have $s_{\Omega^{\circ}}=\rho g(\phi)$, where $g(\phi)>0$ as $0 \in \operatorname{int} \Omega$. So $s_{\Omega^{\circ}}$ is $C^{k}$ iff $g$ is $C^{k}$. Since $g \neq 0$, this is equivalent to the fact that $1 / g$ is $C^{k}$. So if $s_{\Omega^{\circ}}$ is $C^{k}$, then $x(\phi)=\cos \phi / g(\phi), y(\phi)=\sin \phi / g(\phi)$ is a $C^{k}$-parametrization of the boundary $\partial \Omega$, and $x_{\phi}^{\prime 2}+y_{\phi}^{\prime 2}=\left(g^{2}+g^{\prime 2}\right) / g^{4}>0$. 
$(i) \Rightarrow($ iii $)$. Consider a $C^{k}$-parametrization $(x(s), y(s))$ of $\partial \Omega,{x_{s}^{\prime}}^{2}+y_{s}^{\prime 2} \neq 0$. In the (classic) polar coordinates $(\rho, \phi)$ we have the parametrization $(\rho(s), \phi(s))$, which is again $C^{k}$, since $0 \in \operatorname{int} \Omega$. We know that $s_{\Omega^{\circ}}(x(s), y(s)) \equiv 1$, so $g(\phi(s)) \equiv 1 / \rho(s)$. Since $\phi_{s}^{\prime}=\left(x y_{s}^{\prime}-x_{s}^{\prime} y\right) /\left(x^{2}+y^{2}\right)$, we have $\phi_{s}^{\prime} \in C^{k-1}, \phi \in C^{k}$, and $\phi_{s}^{\prime} \neq 0$ as $0 \in \operatorname{int} \Omega$. So the function $g(\phi)$ must be $C^{k}$ by the inverse function theorem.

Proposition 2.6. For any $1 \leq p \leq \infty$ and integer $k \geq 1$, the following statements are equivalent:

(i) the boundary of $\Omega$ is a regular $W_{p}^{k}$-curve ${ }^{2}$;

(ii) $\cos _{\Omega}$ and $\sin _{\Omega}$ are $W_{p}^{k}$ functions (on $\mathbb{R} / 2 \mathbb{S} \mathbb{Z}$ );

(iii) $s_{\Omega^{\circ}}(\cos \phi, \sin \phi)$ is $W_{p}^{k}$ function (on $\left.\mathbb{R} / 2 \pi \mathbb{Z}\right)$.

Proof. $(i) \Rightarrow(i i)$. Let the boundary $\partial \Omega$ be given as $(x(s), y(s))$, where $x, y \in W_{p}^{k}\left[s_{0} ; s_{1}\right]$, and ${x_{s}^{\prime}}^{2}+{y_{s}^{\prime}}^{2} \geq C>0$ for some constant $C>0$. Consider first the case $k=1$. In this case, $s_{\theta}^{\prime}(\theta)=1 / \theta_{s}^{\prime}(s(\theta)) \leq 1 / C$, so $s^{\prime}(\theta)$ is a bounded function and $s(\theta)$ is Lipschitz continuous. Moreover, $s^{\prime}$ vanishes on a set of zero measure. Hence the derivative $\cos _{\Omega}^{\prime} \theta=x_{s}^{\prime}(s(\theta)) s^{\prime}(\theta)$ is defined for a.e. $\theta$, since $\cos _{\Omega} \theta=x(s(\theta))$. Therefore, if $p<\infty$ we have

$$
\int_{\theta\left(s_{0}\right)}^{\theta\left(s_{1}\right)}\left|\cos _{\Omega}^{\prime} \theta\right|^{p} \mathrm{~d} \theta=\int_{\theta\left(s_{0}\right)}^{\theta\left(s_{1}\right)}\left|x_{s}^{\prime}(\theta(s))\right|\left(s^{\prime}(\theta)\right)^{p} \mathrm{~d} \theta \leq \frac{1}{C^{p-1}} \int_{\theta\left(s_{0}\right)}^{\theta\left(s_{1}\right)}\left|x_{s}^{\prime}(\theta(s))\right| s^{\prime}(\theta) \mathrm{d} \theta=\frac{1}{C^{p-1}}\left\|x_{s}^{\prime}\right\|_{p} .
$$

In the case $p=\infty$, we have

$$
\left|\cos _{\Omega}^{\prime} \theta\right|=\left|x_{s}^{\prime}(\theta(s))\right| s^{\prime}(\theta) \leq \frac{1}{C}\left|x_{s}^{\prime}(\theta(s))\right| .
$$

Hence, in the both cases, $\cos _{\Omega} \in W_{p}^{1}\left[\theta\left(s_{0}\right) ; \theta\left(s_{1}\right)\right]$. Similarly, $\sin _{\Omega} \in W_{p}^{1}\left[\theta\left(s_{0}\right) ; \theta\left(s_{1}\right)\right]$.

Now, we consider the case $k \geq 2$. In this case, $\partial \Omega \in C^{k-1}$. Therefore, $\theta(s)$ is a $C^{k-1}$ function and $s(\theta)$ is $C^{k-1}$ too. Moreover, $\theta_{s}^{\prime}$ is a continuous function separated from 0 , so $s(\theta)$ is a bi-Lipschitzian function. Hence, $\theta_{s}^{\prime}(s(\theta))$ is $W_{p}^{k-1}$, since $\theta_{s}^{\prime}(s)$ is $W_{p}^{k-1}$. Moreover, $0<C \leq \theta_{s}^{\prime} \leq C^{\prime}$ for some constants $C^{\prime} \geq C>0$, and the function $a \mapsto 1 / a$ is smooth on $\left[C ; C^{\prime}\right]$. Hence, $s^{\prime}(\theta)=1 / \theta^{\prime}(s(\theta)) \in W_{p}^{k-1}$. Therefore, $s(\theta)$ is $W_{p}^{k}$. It remains to compute

$$
\frac{d^{k}}{\mathrm{~d} \theta^{k}} \cos _{\Omega} \theta=x^{(k)}(s(\theta))\left(s^{\prime}(\theta)\right)^{k}+x_{s}^{\prime}(s(\theta)) s^{(k)}(\theta)+G(\theta)
$$

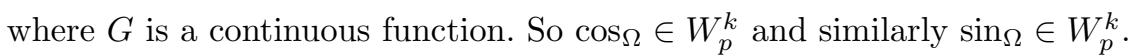

The other proofs $(i i) \Rightarrow(i),(i) \Rightarrow($ iii $)$, and $($ iii $) \Rightarrow(i)$ are similar to the corresponding proofs for Proposition 2.5.

Remark 2.7. Suppose that an open interval $l$ on $\partial \Omega$ has smoothness $W_{p}^{k}$ (or $C^{k}$ ). Then the functions $\cos _{\Omega}$ and $\sin _{\Omega}$ have the same smoothness while the point $P_{\theta}$ moves along $l$.

We already know, that if $\partial \Omega$ is $C^{1}$ then the function $\theta^{\circ}(\theta)$ is continuous. Let us extend this property:

Corollary 2.8. If $\partial \Omega$ is a regular $C^{k}$ (or $W_{p}^{k}$ ) curve for $k \geq 2$ and $1 \leq p \leq+\infty$, then the function $\theta^{\circ}(\theta)$ is $C^{k-1}\left(\right.$ or $\left.W_{p}^{k-1}\right)$ and

$$
\frac{\mathrm{d} \theta^{\circ}}{\mathrm{d} \theta}=\cos _{\Omega}^{\prime} \theta \sin _{\Omega}^{\prime \prime} \theta-\sin _{\Omega}^{\prime} \theta \cos _{\Omega}^{\prime \prime} \theta
$$

\footnotetext{
${ }^{2}$ The term "regular $W_{p}^{k}$-curve" means that there exists a $W_{p}^{k}$ parametrization $(x(s), y(s))$ of the curve such that $x_{s}^{\prime 2}+y_{s}^{\prime 2}$ is separated from 0 for a.e. $s$.
} 
The curvature $\kappa$ of $\partial \Omega$ at the point $\left(\cos _{\Omega} \theta, \sin _{\Omega} \theta\right)$ is given by the formula

$$
\kappa(\theta)=\left(\cos _{\Omega^{\circ}}^{2} \theta^{\circ}+\sin _{\Omega^{\circ}}^{2} \theta^{\circ}\right)^{-3 / 2} \frac{\mathrm{d} \theta^{\circ}}{\mathrm{d} \theta} .
$$

Proof. The pair $\left(\cos _{\Omega}^{\prime} \theta, \sin _{\Omega}^{\prime} \theta\right)$ is a $C^{k-1}$ (or $\left.W_{p}^{k-1}\right)$ parametrization of $\partial \Omega^{\circ}$. Using the inverse polar change of coordinates (see Sect. 2.2), we obtain formula (2.5). Using it, we see that $\theta^{\circ \prime}(\theta)$ is $C^{k-2}$ (or $W_{p}^{k-2}$ ) and $\theta^{\circ}(\theta)$ is $C^{k-1}$ (or $W_{p}^{k-1}$ ).

The curvature can be computed in a standard way: put $\gamma(\theta)=(\cos \Omega, \sin \Omega)$, then $\gamma^{\prime}=\left(-\sin _{\Omega^{\circ}} \theta^{\circ}, \cos _{\Omega^{\circ}} \theta^{\circ}\right)$ and $\gamma^{\prime \prime}=-\gamma \mathrm{d} \theta^{\circ} / \mathrm{d} \theta$. It remains to compute $\kappa=\left(\gamma^{\prime} \times \gamma^{\prime \prime}\right) /\left|\gamma^{\prime}\right|^{3}$.

\section{Finsler GeOMetry on the Lobachevsky HyPERBolic Plane}

Consider the following control system on the upper half-plane $L^{2}=\left\{(x, y) \in \mathbb{R}^{2} \mid y>0\right\}$ :

$$
\left\{\begin{array}{l}
\dot{x}=y u_{1} \\
\dot{y}=y u_{2}
\end{array} ;\right.
$$

where the 2 -dimensional control $u=\left(u_{1}, u_{2}\right)$ belongs to a convex compact set $\Omega$ with $0 \in \operatorname{int} \Omega$. Solutions of the time minimizing problem $T \rightarrow \inf$ for this system are Finsler geodesics on $L^{2}$, where the length of a tangent vector $(\xi, \eta) \in T_{(x, y)} L^{2}$ is given by ${ }^{3} y \mu_{\Omega}(\xi, \eta)$. Alternatively, we can consider this problem as a left-invariant Finsler geometry on the Lie group $\operatorname{Aff}(\mathbb{R})$ of proper affine transformations of the line $\mathbb{R}$. When $\Omega$ is a unit circle, we have the classical hyperbolic plane with Lobachevskian geometry.

In this section we show an easy way of constructing Finsler geodesics on $L^{2}$ by the convex trigonometry. Using the Pontryagin maximum principle (PMP), we get the following generalized Hamiltonian:

$$
\mathcal{H}=p y u_{1}+q y u_{2}
$$

where $p$ and $q$ are adjoint variables. Maximum of $\mathcal{H}$ in $u \in \Omega$ can be written very conveniently in terms of the support function $s_{\Omega}$ of the set $\Omega$ :

$$
H=\max _{u \in \Omega} \mathcal{H}=s_{\Omega}(p y, q y) .
$$

Denote for short the arguments of $s_{\Omega}$ by $h_{1}=p y$ and $h_{2}=q y$. Obviously $\frac{\mathrm{d}}{\mathrm{d} t} H=0$ and $H=$ const. We claim that the case $H=0$ is not possible. Indeed, if $H=0$, then $h_{1}=h_{2}=0$. Since $y>0$, we get $p=q=0$, which contradicts to the Pontryagin maximum principle.

So $H>0$. Consequently the point $\left(h_{1}, h_{2}\right)$ moves along the boundary of the polar set $\Omega^{\circ}$ stretched by $H$ times:

$$
\left(h_{1}, h_{2}\right) \in H \partial \Omega^{\circ} .
$$

Let us now find the "velocity" of the point $\left(h_{1}, h_{2}\right)$. Substituting $\dot{p}=-\mathcal{H}_{x}^{\prime}=0$ and $\dot{q}=-\mathcal{H}_{y}^{\prime}=-p u_{1}-q u_{2}$, we get

$$
\dot{h}_{1}=h_{1} u_{2}, \quad \dot{h}_{2}=-h_{1} u_{1},
$$

\footnotetext{
${ }^{3}$ Here $\mu_{\Omega}=s_{\Omega}$ 。 denotes the Minkowski function of $\Omega$.
} 
and control can be found from the Pontryagin maximum condition $h_{1} u_{1}+h_{2} u_{2} \rightarrow \max _{u \in \Omega}$. Equations on $\dot{h}_{1}$ and $\dot{h}_{2}$ do not depend on the shape of $\Omega$, but solutions to the Pontryagin maximum condition highly depend on the shape of $\Omega$.

Nonetheless, extremals can be found simultaneously for all $\Omega$ by machinery of the convex trigonometry. Let us use the generalized polar change of coordinates (see Sect. 2.2):

$$
h_{1}=H \cos _{\Omega^{\circ}} \theta^{\circ} \quad \text { and } \quad h_{2}=H \sin _{\Omega^{\circ}} \theta^{\circ},
$$

since $H=s_{\Omega}\left(h_{1}, h_{2}\right)$. Moreover, if $\left(u_{1}, u_{2}\right)$ is an optimal control, then $h_{1} u_{1}+h_{2} u_{2}=H$ and $u \in \partial \Omega$. Hence, using the generalized Pythagorean identity (see Sect. 2.2), we get

$$
u_{1}=\cos _{\Omega} \theta \quad \text { and } \quad u_{2}=\sin _{\Omega} \theta
$$

for an angle $\theta \leftrightarrow \theta^{\circ}$.

Let us emphasize that the optimal control $u=\left(\cos _{\Omega} \theta, \sin _{\Omega} \theta\right)$ can be easily recovered from the angle $\theta^{\circ}(t)$ by the relation $\theta \leftrightarrow \theta^{\circ}$. Alternatively, we may use the differentiation formulae (see Sect. 2.2):

$$
\dot{\theta^{\circ}} u_{1}=\dot{\theta^{\circ}} \sin _{\Omega^{\circ}}^{\prime} \theta^{\circ}=\frac{\mathrm{d}}{\mathrm{d} t} \sin _{\Omega^{\circ}} \theta^{\circ} \quad \text { and } \quad \dot{\theta^{\circ}} u_{2}=-\dot{\theta^{\circ}} \cos _{\Omega^{\circ}}^{\prime} \theta^{\circ}=-\frac{\mathrm{d}}{\mathrm{d} t} \cos \Omega^{\circ} \theta^{\circ} \text {. }
$$

Thus we find $u_{1}$ and $u_{2}$ dividing by $\dot{\theta^{\circ}}$.

So the last thing we need is to find $\theta^{\circ}$. Let us compute the derivative of $\theta^{\circ}$. According to the inverse polar change of coordinates (see Sect. 2.2) for a.e. $t$ we have

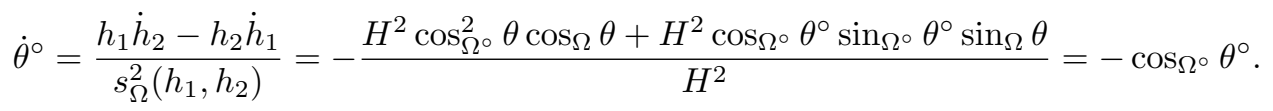

First, consider the case $p \neq 0$. We have

$$
y=\frac{h_{1}}{p}=\frac{H}{p} \cos _{\Omega^{\circ}} \theta^{\circ} .
$$

Let us find $x$. Since $\dot{\theta}^{\circ}=-\cos \Omega^{\circ} \theta^{\circ}$, the derivative $\dot{\theta}^{\circ}$ is continuous. Hence $\theta^{\circ} \in C^{2}$. Thus, the functions $\cos _{\Omega^{\circ}} \theta^{\circ}(t)$ and $\sin _{\Omega^{\circ}} \theta^{\circ}(t)$ are Lipschitz continuous (see Sect. 2.2), and using (3.1) we get

$$
\dot{x}=y u_{1}=\frac{H}{p} \cos _{\Omega^{\circ}} \theta^{\circ} \cos _{\Omega} \theta=-\frac{H}{p} \cos _{\Omega} \theta \dot{\theta^{\circ}}=-\frac{H}{p} \frac{\mathrm{d}}{\mathrm{d} t} \sin _{\Omega^{\circ}} \theta^{\circ} ;
$$

since $\left(\cos _{\Omega^{\circ}} \theta^{\circ}\right)^{\prime}=-\sin _{\Omega} \theta$ and $\left(\sin _{\Omega^{\circ}} \theta^{\circ}\right)^{\prime}=\cos _{\Omega} \theta$ by the differentiation formulae (see Sect. 2.2). Recall that $H=$ const and $p=$ const, so

$$
x=x_{0}-\frac{H}{p} \sin _{\Omega^{\circ}} \theta^{\circ} .
$$

Thus, for the case $p \neq 0$ we have proved that Finsler geodesics on the Lobachevsky plane coincide with parts of boundary of the polar set $\Omega^{\circ}$, which is stretched $\frac{H}{|p|}$ times, rotated $\pm 90^{\circ}$ (depending on the sign of $p$ ) and moved horizontally to the distance $x_{0}$. These geodesics are naturally called horizontal.

A natural parametrization of geodesics is given by solutions of the equation

$$
\dot{\theta^{\circ}}=-\cos \Omega^{\circ} \theta^{\circ} .
$$



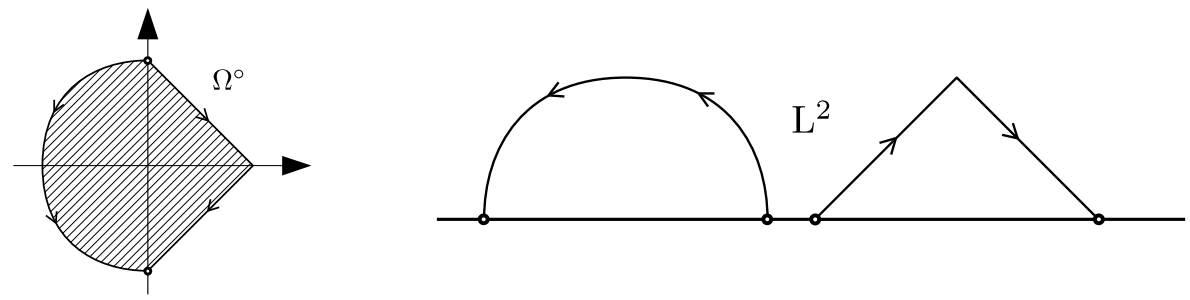

Figure 1. Examples of the polar set $\Omega^{\circ}$ and Finsler geodesics on $L^{2}$.

The topological structure of solutions to this equation is very simple. The point $Q_{\theta^{\circ}}=\left(\cos _{\Omega^{\circ}} \theta^{\circ}, \sin _{\Omega^{\circ}} \theta^{\circ}\right)$ moves along $\partial \Omega^{\circ}$. The intersection points of $\partial \Omega^{\circ}$ with the vertical axis are fixed points. In the right half-plane, $Q_{\theta^{\circ}}$ moves clockwise, and in the left half-plane, $Q_{\theta^{\circ}}$ moves counterclockwise (see Fig. 1). Note that if the set $\Omega$ is not symmetric w.r.t. the origin, then the distance function on $L^{2}$ is not $\operatorname{symmetric} t$ oo, $\operatorname{dist}(A, B) \neq \operatorname{dist}(B, A)$. So, if $p \neq 0$, then geodesics going to the left and to the right are different. In the case, when the set $\Omega$ is symmetric, $L^{2}$ becomes a metric space and geodesics going to the left and to the right are represented by the same curves.

Equation (3.2) can be explicitly integrated in many cases. For example, if $\Omega$ is a convex polygon, then $\Omega^{\circ}$ is a polygon too and the functions $\cos _{\Omega^{\circ}} \theta^{\circ}$ and $\sin _{\Omega^{\circ}} \theta^{\circ}$ are linear in $\theta^{\circ}$ while the point $Q_{\theta^{\circ}}$ moves along an edge of $\Omega^{\circ}$. So in this case the equation $\dot{\theta}^{\circ}=-\cos \Omega^{\circ} \theta^{\circ}$ on an edge takes the form $\dot{\theta}^{\circ}=a \theta^{\circ}+b$ and can be integrated easily. If $\Omega$ is an ellipse (see Sect. 2.3), then equation (3.2) takes the form

$$
\dot{s}=\frac{-a \cos s-x_{0}}{a b-y_{0} a \sin s+x_{0} b \cos s},
$$

which can be integrated explicitly by elementary functions.

Now consider the last case $p=0$. We have $h_{1} \equiv 0$ and $h_{2}=$ const $\neq 0$. If $h_{2}>0$, then the optimal control $u=\left(u_{1}, u_{2}\right) \in \partial \Omega$ has maximal second coordinate $u_{2}$, and $u$ is the upper point of $\Omega$ (if it is unique) or moves arbitrarily along the upper edge of $\Omega$. The case $h_{2}<0$ is similar. It is easy to see that, in both cases, the described control is optimal, since the coordinate $y$ takes maximal (or minimal) possible value at any given time $T$. These geodesics are naturally called vertical.

All vertical geodesics moving up from a given point $\left(x_{0}, y_{0}\right) \in L^{2}$ form a domain in $L^{2}$ bounded by parts of two lines $\left(y-y_{0}\right) /\left(x-x_{0}\right)=u_{2}^{0} / u_{1}^{0}, y \geq y_{0}$, which appear when the control $u$ is constant, $u(t)=\left(u_{1}^{0}, u_{2}^{0}\right)$, and sits at the left (or right) end of the upper edge of $\Omega$. The similar fact holds for vertical geodesics moving down.

Summarizing, we get the following full description of geodesics in the Finsler problem on the Lobachevsky hyperbolic plane:

Theorem 3.1. There are two types of geodesics in the sub-Finsler problem on the Lobachevsky hyperbolic plane (3.1):

(h) Horizontal geodesics come from the polar set $\Omega^{\circ}$. To obtain a horizontal geodesic one should (i) rotate $\Omega^{\circ}$ by $\pm 90^{\circ}$, (ii) stretch it $\lambda>0$ times, (iii) take the upper part of the boundary, and (iv) move this part horizontally to any distance. If $\Omega^{\circ}$ was rotated clockwise then the motion on the geodesic is counterclockwise and vice versa (see Fig. 1).

$(v)$ To obtain a vertical geodesic moving up one should choose an arbitrary measurable control $u(t) \in \Omega$ having maximal possible $u_{2}$-coordinate in $\Omega$ for a.e. $t$. Then the corresponding vertical geodesic starting at $\left(x_{0}, y_{0}\right)$ has the following form:

$$
x(t)=x_{0}+y_{0} \int_{0}^{t} e^{u_{2} s} u_{1}(s) \mathrm{d} s ; \quad y(t)=y_{0} e^{u_{2} t} .
$$


Vertical geodesics moving down are constructed in the same way by taking $u(t) \in \Omega$ having minimal possible $u_{2}$-coordinate in $\Omega$ for all $t$.

\section{SECOND ORDER ODEs CONTAINING FunCtions OF CONVEX TRIGONOMETRY}

A lot of problems considered below can be reduced to solving a differential inclusion containing functions $\cos \Omega$ and $\sin _{\Omega}$ of the following form ${ }^{4}$ :

$$
\ddot{\theta}^{\circ}(t) \in-\mathcal{U}^{\prime}\left(\theta^{\circ}(t)\right) \text { for a.e. } t
$$

where $\theta^{\circ}(t) \in W_{\infty}^{2}$ (i.e., $\dot{\theta}^{\circ}$ is a Lipschitz continuous functions), $\mathcal{U}\left(\theta^{\circ}\right)=f\left(\cos _{\Omega^{\circ}} \theta^{\circ}, \sin _{\Omega^{\circ}} \theta^{\circ}\right), f(p, q)$ is a sufficiently smooth function, and the symbol $\mathcal{U}^{\prime}$ denotes

$$
\mathcal{U}^{\prime}\left(\theta^{\circ}\right)=\left\{-f_{p} \sin _{\Omega} \theta+f_{q} \cos _{\Omega} \theta \quad \text { for all } \theta \leftrightarrow \theta^{\circ}\right\} .
$$

We use the previous notation, since $\mathcal{U}\left(\theta^{\circ}\right)$ has derivative for a.e. $\theta^{\circ}$ which is equal to $-f_{p} \sin _{\Omega} \theta+f_{q} \sin _{\Omega} \theta$ by the differentiation formulae (see Sect. 2.2). The derivative $\mathcal{U}^{\prime}\left(\theta^{\circ}\right)$ certainly exists if there exists a unique $\theta \leftrightarrow \theta^{\circ}$ (up to the period $2 \mathbb{S}^{\circ}=2 \mathbb{S}\left(\Omega^{\circ}\right)$ ). In the opposite case, $\mathcal{U}\left(\theta^{\circ}\right)$ has left and right derivatives, and the interval between them we denoted by $\mathcal{U}^{\prime}\left(\theta^{\circ}\right)$. In the last case, the left and right derivatives coincide iff $d f\left(\cos _{\Omega^{\circ}} \theta^{\circ}, \sin _{\Omega^{\circ}} \theta^{\circ}\right) \perp$ $\left(\cos \Omega_{\Omega^{\circ}} \theta^{\circ}, \sin _{\Omega^{\circ}} \theta^{\circ}\right)$.

Usually, if $\mathcal{U}^{\prime}\left(\theta^{\circ}\right)$ consists of a unique element for some $\theta^{\circ}, \mathcal{U}^{\prime}\left(\theta^{\circ}\right)=\{a\}$, we will simply write $\mathcal{U}^{\prime}\left(\theta^{\circ}\right)=a$ for short.

Proposition 4.1. For any given initial data $\theta_{0}^{\circ}, \theta_{1}^{\circ} \in \mathbb{R}$, there exists a solution $\hat{\theta}^{\circ}(t)$ to $(4.1)$ satisfying $\hat{\theta}^{\circ}(0)=\theta_{0}^{\circ}$ and $\dot{\hat{\theta}}^{\circ}(0)=\theta_{1}^{\circ}$ and defined for all $t \in \mathbb{R}$.

Proof. The multivalued function $\mathcal{U}^{\prime}$ is upper semicontinuous at any point $\theta^{\circ}$ in the following sense: for any $\theta^{\circ} \in \mathbb{R}$ and $\varepsilon>0$ there exists $\delta>0$ such that $\mathcal{U}^{\prime}\left(\tilde{\theta}^{\circ}\right)$ belongs to the $\varepsilon$-neighborhood of $\mathcal{U}^{\prime}\left(\theta^{\circ}\right)$ for any $\tilde{\theta}^{\circ} \in \mathbb{R}$ in the $\delta$-neighborhood of $\theta^{\circ}$ (this fact follows immediately from the differentiation formulae in Sect. 2.2). Moreover, the set $\mathcal{U}^{\prime}\left(\theta^{\circ}\right)$ is a non empty convex compactum for any $\theta^{\circ}$. Hence, for any initial data $\hat{\theta}^{\circ}(0)=\theta_{0}^{\circ}, \dot{\hat{\theta}}^{\circ}(0)=\theta_{1}^{\circ}$, there exists a solution to (4.1) in a neighborhood of $t=0$ by Filippov's theorem (see [26]). Moreover, any solution can be extended to the whole line $t \in \mathbb{R}$, since the function $\mathcal{U}^{\prime}$ is bounded.

Nonetheless, uniqueness of solution to (4.1) (with given initial data) may fail. Despite the fact that $\mathcal{U}\left(\theta^{\circ}\right)$ has derivative w.r.t. $\theta^{\circ}$ for a.e. $\theta^{\circ}$, existence of the derivative of function $\mathcal{U}\left(\hat{\theta}^{\circ}(t)\right)$ w.r.t. $t$ needs additional verification.

In the present section we will prove some general facts about differential inclusion (4.1). It appears that uniqueness may fail only in very specific situations. Moreover, these situations correspond to singular extremals in optimal control problems considered below.

\subsection{First integral of energy}

Theorem 4.2. The function $\mathbb{E}=\frac{1}{2}\left(\dot{\theta}^{\circ}\right)^{2}+\mathcal{U}\left(\theta^{\circ}\right)$ is a first integral for (4.1), i.e., $\mathbb{E}$ is constant along any solution $\hat{\theta}^{\circ}(t)$ to (4.1). Moreover, if $\tilde{\theta}^{\circ}(t)$ is an arbitrary $C^{1}$-function (on an interval), $\dot{\tilde{\theta}}^{\circ}(t) \neq 0$ for all $t$, and $\mathbb{E}$ is constant along $\tilde{\theta}^{\circ}$, then $\tilde{\theta}^{\circ}$ belongs to $W_{\infty}^{2}$ on the interval and it is a solution to (4.1).

\footnotetext{
${ }^{4}$ Results similar to those obtained in the present section are also fulfilled for the inclusion $\ddot{\theta}(t) \in-\mathcal{U}^{\prime}(\theta(t))$ by the bipolar theorem.
} 
Proof. We start with the first part of the theorem. Denote $Z=\left\{t: \dot{\hat{\theta}}^{\circ}(t)=0\right\}$. For a.e. $\theta^{\circ}$ there exists a unique $\theta \leftrightarrow \theta^{\circ}$, since $\partial \Omega^{\circ}$ has a countable number of corners at most. Hence, for a.e. $t \notin Z$ there exists a unique $\hat{\theta}(t) \leftrightarrow \hat{\theta}^{\circ}(t)$ and the set $\mathcal{U}^{\prime}\left(\hat{\theta}^{\circ}(t)\right)$ contains a unique element, which must coincide with $-\ddot{\hat{\theta}}^{\circ}$. Therefore, the function $\mathcal{U}\left(\hat{\theta}^{\circ}(t)\right)$ has derivative for a.e. $t \notin Z$, and

$$
\dot{\mathbb{E}}=\ddot{\hat{\theta}}^{\circ}(t) \dot{\hat{\theta}}^{\circ}(t)+\dot{\hat{\theta}}^{\circ}(t) \mathcal{U}^{\prime}\left(\hat{\theta}^{\circ}(t)\right)=0 \text { for a.e. } t \notin Z \text {. }
$$

It remains to consider the case $t \in Z$. Since $\hat{\theta}^{\circ} \in W_{\infty}^{2}$, there exists $\ddot{\hat{\theta}}^{\circ}(t)$ for a.e. $t \in Z$. Moreover, for all $t \in Z$, $\hat{\theta}^{\circ}(t+\tau)-\hat{\theta}^{\circ}(t)=o(\tau)$. Hence, $\frac{\mathrm{d}}{\mathrm{d} t} \mathcal{U}\left(\hat{\theta}^{\circ}(t)\right)=0$ for all $t \in Z$, since the functions cos$\Omega_{\Omega^{\circ}}$ and $\sin _{\Omega^{\circ}}$ are Lipschitz continuous. Therefore, we have

$$
\dot{\mathbb{E}}=\ddot{\hat{\theta}}^{\circ}(t) \dot{\hat{\theta}}^{\circ}(t)+\left.\frac{\mathrm{d}}{\mathrm{d} \tau}\right|_{\tau=0} \mathcal{U}\left(\hat{\theta}^{\circ}(t+\tau)\right)=0 \quad \text { for a.e. } t \in Z .
$$

Let us prove the second part. Consider a $C^{1}$-function $\tilde{\theta}^{\circ}$ such that $\dot{\tilde{\theta}}^{\circ}(t) \neq 0$ for all $t$ on an interval. Assume that $\dot{\tilde{\theta}}^{\circ}(t)>0$ for all $t$ (the case $\dot{\tilde{\theta}}^{\circ}(t)<0$ for all $t$ is similar). In this case, $\dot{\tilde{\theta}}^{\circ}=\sqrt{2}\left(\mathbb{E}-\mathcal{U}\left(\tilde{\theta}^{\circ}\right)\right)^{1 / 2}$. Therefore, $\dot{\tilde{\theta}^{\circ}}$ is locally Lipschitz continuous and $\tilde{\theta}^{\circ} \in W_{\infty, \text { loc }}^{2}$. Hence $0=\dot{\mathbb{E}}=\dot{\tilde{\theta}}^{\circ} \ddot{\tilde{\theta}}^{\circ}+\mathcal{U}^{\prime}\left(\tilde{\theta}^{\circ}\right) \dot{\tilde{\theta}}^{\circ}$. So $\ddot{\tilde{\theta}}^{\circ}=-\mathcal{U}^{\prime}\left(\tilde{\theta}^{\circ}\right)$ for a.e. $t$, since $\dot{\tilde{\theta}}^{\circ}(t) \neq 0$. It remains to say that $\mathcal{U}^{\prime}$ is a bounded function, therefore $\tilde{\theta}^{\circ} \in W_{\infty}^{2}$.

\subsection{Uniqueness of solution}

Let us now fix some initial data $\theta_{0}^{\circ}, \theta_{1}^{\circ} \in \mathbb{R}$ :

$$
\theta^{\circ}(0)=\theta_{0}^{\circ} ; \quad \dot{\theta}^{\circ}(0)=\theta_{1}^{\circ} .
$$

If $\partial \Omega^{\circ}$ is sufficiently smooth, then uniqueness of solution with the given initial data must hold due to the classical Picard theorem.

Proposition 4.3. Let $l$ be an open segment on $\partial \Omega^{\circ}$ and $\left(\cos \Omega_{\Omega^{\circ}} \theta_{0}^{\circ}, \sin _{\Omega^{\circ}} \theta_{0}^{\circ}\right) \in l$. If $l$ is $W_{\infty}^{2}$, then there exists a unique solution $\hat{\theta}^{\circ}(t)$ to (4.1) in a neighborhood of $t=0$ satisfying initial data (4.2).

Proof. Indeed, in this case, $\cos _{\Omega^{\circ}}$ and $\sin _{\Omega^{\circ}}$ are $W_{\infty}^{2}$-functions by Proposition 2.6 (see Rem. 2.7). Hence $\mathcal{U} \in W_{\infty}^{2}$ and $\mathcal{U}^{\prime} \in W_{\infty}^{1}$, i.e., $\mathcal{U}^{\prime}$ is a (single-valued) Lipschitz continuous function. Therefore there exists a unique solution by the Picard theorem.

Consequently, absence of uniqueness may be present only at points, where the boundary $\partial \Omega^{\circ}$ does not have $W_{\infty}^{2}$-smoothness. For example, if $\Omega^{\circ}$ is a polygon. Another example is the following one. If $1<p<2$, then the boundary of the set $\left\{x^{p}+y^{p} \leq 1\right\} \subset \mathbb{R}^{2}$ is $W_{q}^{2}$-smooth for $q<1 /(2-p)$, but is not $W_{q}^{2}$-smooth for $q>1 /(2-p)$. So the previous proposition does not work here, and we will show absence of uniqueness for solution of Pontryagin maximum principle in these cases for all left-invariant sub-Finsler problems on all unimodular 3D Lie groups in Section 5 .

Let us now investigate the non-uniqueness phenomenon.

Proposition 4.4. Suppose that $\theta_{1}^{\circ} \neq 0$. Then, there exists a unique solution $\hat{\theta}^{\circ}(t)$ to $(4.1)$ in a neighborhood of $t=0$ satisfying initial data (4.2). Moreover,

$$
\sqrt{2} t=\operatorname{sgn} \theta_{1}^{\circ} \int_{\theta_{0}^{\circ}}^{\hat{\theta}^{\circ}(t)} \frac{\mathrm{d} \theta^{\circ}}{\sqrt{\mathbb{E}-\mathcal{U}\left(\theta^{\circ}\right)}},
$$


where $\mathbb{E}=\frac{1}{2}\left(\theta_{1}^{\circ}\right)^{2}+\mathcal{U}\left(\theta_{0}^{\circ}\right)$.

Proof. Obviously, $\left|\dot{\hat{\theta}}^{\circ}(t)\right|=\sqrt{2}\left(\mathbb{E}-\mathcal{U}\left(\hat{\theta}^{\circ}(t)\right)\right)^{1 / 2}$ by Theorem 4.2. Since $\mathcal{U}\left(\theta^{\circ}\right)<\mathbb{E}$ for $\theta^{\circ}$ in a neighborhood of $\theta_{0}^{\circ}$, we have

$$
\frac{\dot{\hat{\theta}^{\circ}}(t)}{\sqrt{\mathbb{E}-\mathcal{U}\left(\hat{\theta}^{\circ}(t)\right)}}=\operatorname{sgn} \theta_{1}^{\circ} \sqrt{2}
$$

for $t$ in a neighborhood of 0 . Hence, (4.3) must hold. By the inverse function theorem, equation (4.3) has a unique solution, since derivative (w.r.t. $\left.\theta^{\circ}\right)$ of the right-hand side is equal to $\left(\mathbb{E}-\mathcal{U}\left(\theta^{\circ}\right)\right)^{-1 / 2}$, which is continuous and does not vanish in a neighborhood of $\theta_{0}^{\circ}$.

Hence, uniqueness may fail only at points, where $\dot{\theta}^{\circ}=0$. Moreover, even if $\dot{\theta}^{\circ}=0$ this does not guarantee absence of uniqueness (as for example in the case $\partial \Omega^{\circ} \in W_{\infty}^{2}$ ).

Proposition 4.5. Let $\theta_{0}^{\circ} \in \mathbb{R}$ and $\theta_{1}^{\circ}=0$. If $0 \notin \mathcal{U}^{\prime}\left(\theta_{0}^{\circ}\right)$, then there exists a unique solution $\hat{\theta}^{\circ}(t)$ to $(4.1)$ in a neighborhood of $t=0$ satisfying initial data (4.2). This solution is even $\hat{\theta}^{\circ}(t)=\hat{\theta}^{\circ}(-t)$ for $t$ lying in the neighborhood and satisfies

$$
\sqrt{2}|t| \operatorname{sgn} \mathcal{U}^{\prime}\left(\theta_{0}^{\circ}\right)=\int_{\theta_{0}^{\circ}}^{\hat{\theta}^{\circ}(t)} \frac{\mathrm{d} \theta^{\circ}}{\sqrt{\mathbb{E}-\mathcal{U}\left(\theta^{\circ}\right)}} .
$$

Proof. Since $\theta_{1}^{\circ}=0$, we have $\mathbb{E}=\mathcal{U}\left(\theta_{0}^{\circ}\right)$. Let $\mathcal{U}^{\prime}\left(\theta_{0}^{\circ}\right)<0$ (the case $\mathcal{U}^{\prime}\left(\theta_{0}^{\circ}\right)>0$ is similar). Then $\mathcal{U}^{\prime}\left(\theta^{\circ}\right) \subset[-a ;-b]$ in a neighborhood of $\theta_{0}^{\circ}$ for some $a>b>0$. Hence, using (4.1), we obtain $\ddot{\hat{\theta}}^{\circ}(t)>0, \dot{\hat{\theta}}^{\circ}(t)>0$, and $\hat{\theta}^{\circ}(t)>\theta^{\circ}$ for small $t>0$. Thereby

$$
\sqrt{2} t=\int_{\theta_{0}^{\circ}}^{\hat{\theta}^{\circ}(t)} \frac{\mathrm{d} \theta^{\circ}}{\sqrt{\mathbb{E}-\mathcal{U}\left(\theta^{\circ}\right)}} \text { for } t \geq 0 .
$$

This integral has singularity at the left end, but it is finite, since $\mathbb{E}-\mathcal{U}\left(\theta^{\circ}\right)>b \theta^{\circ}$. Put $\hat{\theta}^{\circ}(t)=\theta_{0}^{\circ}+\varphi^{2}, \varphi \geq 0$ :

$$
\sqrt{2} t=\int_{\theta_{0}^{\circ}}^{\theta_{0}^{\circ}+\varphi^{2}} \frac{\mathrm{d} \theta^{\circ}}{\sqrt{\mathbb{E}-\mathcal{U}\left(\theta^{\circ}\right)}} \stackrel{\text { def }}{=} F(\varphi) .
$$

The function $F$ is absolutely continuous, $F(0)=0$, and

$$
\frac{2}{\sqrt{a}} \leq F^{\prime}(\varphi)=\frac{2 \varphi}{\sqrt{\mathbb{E}-\mathcal{U}\left(\theta_{0}^{\circ}+\varphi^{2}\right)}} \leq \frac{2}{\sqrt{b}}
$$

Hence, $F$ is a strictly monotone increasing function, and there exists a unique solution $\varphi=\varphi(t) \geq 0$ to the equation $F(\varphi)=\sqrt{2} t$ for small $t \geq 0$. Moreover, $\varphi(t)$ is a bi-Lipschitz continuous function, and $\hat{\theta}^{\circ}(t)=\theta_{0}^{\circ}+\varphi^{2}(t)$.

For $t \leq 0$, we have

$$
-\sqrt{2} t=\int_{\theta_{0}^{\circ}}^{\hat{\theta}^{\circ}(t)} \frac{\mathrm{d} \theta^{\circ}}{\sqrt{\mathbb{E}-\mathcal{U}\left(\theta^{\circ}\right)}} \text { for } t \geq 0 .
$$

Hence $\hat{\theta}^{\circ}(-t)=\hat{\theta}^{\circ}(t)$. 
The condition $0 \notin \mathcal{U}^{\prime}\left(\theta_{0}^{\circ}\right)$ is equivalent to the following one: the vector $d f\left(\cos _{\Omega^{\circ}} \theta_{0}^{\circ}, \sin _{\Omega^{\circ}} \theta_{0}^{\circ}\right)$ is not null and defines a non-supporting line to $\Omega^{\circ}$ at $\left(\cos _{\Omega^{\circ}} \theta_{0}^{\circ}, \sin _{\Omega^{\circ}} \theta_{0}^{\circ}\right)$.

\subsection{Absence of uniqueness}

So uniqueness may fail only if $\theta_{1}^{\circ}=0$ and $0 \in \mathcal{U}^{\prime}\left(\theta_{0}^{\circ}\right)$. Let us now investigate this situation. Obviously, if $\mathcal{U}\left(\theta^{\circ}\right) \geq \mathcal{U}\left(\theta_{0}^{\circ}\right)$ in a neighborhood of $\theta_{0}^{\circ}$, then a unique solution with $\theta_{1}^{\circ}=0$ is $\hat{\theta}^{\circ}(t) \equiv \theta_{0}^{\circ}$. Indeed, $\left(\dot{\hat{\theta}}^{\circ}(t)\right)^{2} \leq 0$ by Theorem 4.2. It remains to describe solutions for the cases, when $\mathcal{U}\left(\theta^{\circ}\right)<\mathcal{U}\left(\theta_{0}^{\circ}\right)$ in a left or in a right neighborhood ${ }^{5}$ of $\theta_{0}^{\circ}$.

The following theorem describes behaviour of solutions only for $t \geq 0$. The case $t \leq 0$ is similar, since if $\hat{\theta}^{\circ}(t)$ is a solution to (4.1), (4.2) and $\theta_{1}^{\circ}=0$, then $\hat{\theta}^{\circ}(-t)$ is a solution to (4.1), (4.2) as well.

Theorem 4.6. Let $\theta_{0}^{\circ} \in \mathbb{R}, \theta_{1}^{\circ}=0$, and $0 \in \mathcal{U}^{\prime}\left(\theta_{0}^{\circ}\right)$. Put $\mathbb{E}=\mathcal{U}\left(\theta_{0}^{\circ}\right)$. Suppose that $\mathcal{U}\left(\theta^{\circ}\right)<\mathbb{E}$ either $(r)$ in a right, or $(l)$ in a left, or $(b)$ in both left and right neighborhoods of $\theta_{0}^{\circ}$. Then there exists $\tau>0$ with the following properties.

(1) Let $\hat{\theta}^{\circ}(t)$ be a solution to (4.1), (4.2) for $t \geq 0$. Put

$$
t_{+}=\sup \left\{t \geq 0: \hat{\theta}^{\circ}(s)=\theta_{0}^{\circ} \text { for all } s \in[0 ; t]\right\} .
$$

If there exists a solution $\hat{\theta}^{\circ}$ with $t_{+}<\infty$, then $\dot{\hat{\theta}}^{\circ}(t)$ for $t \in\left(t_{+} ; t_{+}+\tau\right)$ is not null ${ }^{6}$, and $\left(\mathbb{E}-\mathcal{U}\left(\theta^{\circ}\right)\right)^{-1 / 2} \in$ $L_{1}\left(\theta_{0}^{\circ}, \theta_{0}^{\circ}+\varepsilon\right)$ for some $\varepsilon \neq 0, \operatorname{sgn} \varepsilon=\operatorname{sgn} \dot{\hat{\theta}}^{\circ}\left(t_{+}+0\right)$. Moreover, for any solution $\hat{\theta}^{\circ}$ with $t_{+}<\infty$, we have

$$
\sqrt{2}\left(t-t_{+}\right) \operatorname{sgn} \dot{\hat{\theta}}^{\circ}\left(t_{+}+0\right)=\int_{\theta_{0}^{\circ}}^{\hat{\theta}^{\circ}(t)} \frac{\mathrm{d} \theta^{\circ}}{\sqrt{\mathbb{E}-\mathcal{U}\left(\theta^{\circ}\right)}} \quad \text { for } \quad t \in\left(t_{+} ; t_{+}+\tau\right] .
$$

(2) If $(r)$ (or $(b))$ and $\left(\mathbb{E}-\mathcal{U}\left(\theta^{\circ}\right)\right)^{-1 / 2} \in L_{1}\left(\theta_{0}^{\circ}, \theta_{0}^{\circ}+\varepsilon\right)$ for some $\varepsilon>0$, then for any $t_{+} \geq 0$ there exists a solution $\hat{\theta}^{\circ}(t)$ to (4.1), (4.2) for $t \geq 0$ such that $\hat{\theta}^{\circ}(t)=\theta_{0}^{\circ}$ for $t \in\left[0 ; t_{+}\right]$, $\dot{\hat{\theta}}^{\circ}(t)$ is positive for $t \in$ $\left(t_{+} ; t_{+}+\tau\right)$, and (4.5) holds. The remaining case $((l)$ or $(b))$ is similar with $\varepsilon<0$.

The geometric meaning of the case $0 \in \mathcal{U}^{\prime}\left(\theta_{0}^{\circ}\right)$ is the following. The constant $\hat{\theta}^{\circ}(t) \equiv \theta_{0}^{\circ}$ is always a solution. But if, for example, $\mathcal{U}\left(\theta^{\circ}\right)<\mathcal{U}\left(\theta_{0}^{\circ}\right)$ in a right neighborhood of $\theta_{0}^{\circ}$ and the following improper integral

$$
\int_{\theta_{0}^{\circ}}^{\theta_{0}^{\circ}+\varepsilon} \frac{\mathrm{d} \theta^{\circ}}{\sqrt{\mathbb{E}-\mathcal{U}\left(\theta^{\circ}\right)}}
$$

is finite for some $\varepsilon>0$, then uniqueness fails. A solution stays at $\theta_{0}^{\circ}$ for an arbitrary time $t_{+} \geq 0$ and then goes to the right for at least $\tau>0$ time. So, there is a 1 -parameter family (determined by the parameter $t_{+} \geq 0$ ) of solutions for $t \geq 0$ defined on $\left[0 ; t_{+}+\tau\right]$. Each solution can then be extended for $t \in[0 ;+\infty)$ by Filippov's theorem. If $\mathcal{U}\left(\theta^{\circ}\right)<\mathcal{U}\left(\theta_{0}^{\circ}\right)$ in a left neighborhood of $\theta_{0}^{\circ}$ and the improper integral is finite for some $\varepsilon<0$, then a solution can go also to the left (and there is another 1-parameter family of solutions for $t \in\left[0 ; t_{+}+\tau\right], t_{+} \geq 0$ ). A similar situation happens for $t \leq 0$.

Proof of Theorem 4.6. Denote $\Theta=\left\{\theta^{\circ} \in \mathbb{R}: \mathcal{U}\left(\theta^{\circ}\right)=\mathbb{E}\right\}$. Obviously, if $\dot{\hat{\theta}}^{\circ}(t)=0$, then $\hat{\theta}^{\circ}(t) \in \Theta$ by Theorem 4.2. Put

$$
\theta_{r}^{\circ}=\inf \left(\Theta \cap\left\{\theta^{\circ}>\theta_{0}^{\circ}\right\}\right) ; \quad \theta_{l}^{\circ}=\sup \left(\Theta \cap\left\{\theta^{\circ}<\theta_{0}^{\circ}\right\}\right) .
$$

\footnotetext{
${ }^{5}$ We omit here some rare cases, when $\mathcal{U}\left(\theta^{\circ}\right) \leq 0$ in right (or left) neighborhood of $\theta_{0}^{\circ}$, but inequality $\mathcal{U}\left(\theta^{\circ}\right)<0$ does not hold in any left (of right) neighborhood of $\theta_{0}^{\circ}$.

${ }^{6}$ Hence, it must be positive in the case $(r)$ and negative in the case $(l)$.
} 
If $(r)$ or $(b)$, then $\theta_{r}^{\circ}>\theta_{0}^{\circ}$, and if $(l)$ or $(b)$, then $\theta_{l}^{\circ}<\theta_{0}^{\circ}$ by the hypothesis of the theorem.

Since $\left|\dot{\hat{\theta}}^{\circ}(t)\right|=\sqrt{2}\left(\mathbb{E}-\mathcal{U}\left(\hat{\theta}^{\circ}(t)\right)\right)^{1 / 2}$ for any solution $\hat{\theta}^{\circ}$ and $\mathcal{U}$ is a bounded function, the derivative $\dot{\hat{\theta}}^{\circ}(t)$ is bounded by the constant $M=\sqrt{2}\left(\mathbb{E}-\min _{\theta} \mathcal{U}\left(\theta^{\circ}\right)\right)^{1 / 2}$, i.e., $\left|\dot{\hat{\theta}}^{\circ}(t)\right| \leq M$ for all $t$. Denote

$$
\tau_{l}=\left(\theta_{0}^{\circ}-\theta_{l}^{\circ}\right) / M, \quad \tau_{r}=\left(\theta_{r}^{\circ}-\theta_{0}^{\circ}\right) / M,
$$

and put $(l) \tau=\tau_{l},(r) \tau=\tau_{r}$, or $(b) \tau=\min \left\{\tau_{l}, \tau_{r}\right\}$. Obviously $\tau>0$.

The proof of item 1 is based on the following key property of $\tau$ : if $\hat{\theta}^{\circ}\left(t_{1}\right)=\hat{\theta}^{\circ}\left(t_{2}\right)=\theta_{0}^{\circ}$ and $\left|t_{2}-t_{1}\right|<\tau$, then $\hat{\theta}^{\circ}(t)=\theta_{0}^{\circ}$ for all $t$ between $t_{1}$ and $t_{2}$. Let us prove this property by contradiction. Let $t_{1}<t_{2}$. Suppose that there exists $s \in\left(t_{1}, t_{2}\right)$ such that $\hat{\theta}^{\circ}(s) \neq \theta_{0}^{\circ}$. Let for example $\hat{\theta}^{\circ}(s)>\theta_{0}^{\circ}$ (this is possible only if $(r)$ or $(b)$ is fulfilled). Put $T=\arg \max _{t \in\left[t_{1} ; t_{2}\right]} \hat{\theta}^{\circ}(t)$. Then $\hat{\theta}^{\circ}(T)>\theta_{0}^{\circ}, \dot{\hat{\theta}}^{\circ}(T)=0$, and $\hat{\theta}^{\circ}(T) \in \Theta$. Therefore, $\hat{\theta}^{\circ}(T) \geq \theta_{r}^{\circ}$ and we have the following contradiction:

$$
\theta_{r}^{\circ}-\theta_{0}^{\circ} \leq \hat{\theta}^{\circ}(T)-\hat{\theta}^{\circ}\left(t_{1}\right) \leq M\left(T-t_{1}\right)<M \tau \leq \theta_{r}^{\circ}-\theta_{0}^{\circ} .
$$

Now we are ready to prove item 1 of the theorem. Suppose that there exists a solution $\hat{\theta}^{\circ}$ with $t_{+}<\infty$. By the definition of $t_{+}$, there exists $s \in\left(t_{+} ; t_{+}+\tau\right)$ such that $\hat{\theta}^{\circ}(s) \neq \theta_{0}^{\circ}$. We claim that if $\hat{\theta}^{\circ}(s)>\theta_{0}^{\circ}$, then $\dot{\hat{\theta}}^{\circ}(t)>0$ for all $t \in\left(t_{+} ; t_{+}+\tau\right)$. Let us prove this claim again by contradiction. Since $\hat{\theta}^{\circ}(s)>\theta_{0}^{\circ},(r)$ or $(b)$ is fulfilled. Moreover, if the claim does not hold, then there exists $T \in\left(t_{+} ; t_{+}+\tau\right)$ such that $\dot{\hat{\theta}}^{\circ}(T)=0$. Therefore, $\hat{\theta}^{\circ}(T) \in \Theta$. Since $T-t_{+}<\tau$, we have $\hat{\theta}^{\circ}(T)=\theta_{0}^{\circ}$. Hence, $\hat{\theta}^{\circ}(t)=\theta_{0}^{\circ}$ for $t \in\left[t_{+} ; T\right]$ by the key property of $\tau$, and we obtain a contradiction with the definition of $t_{+}$. The case $\hat{\theta}^{\circ}(s)<\theta_{0}^{\circ}$ is similar.

So, $\dot{\hat{\theta}}^{\circ}(t) \neq 0$ and it does not change sign for all $t \in\left(t_{+} ; t_{+}+\tau\right)$. Let $\dot{\hat{\theta}}^{\circ}(t)>0$ for all $t \in\left(t_{+} ; t_{+}+\tau\right)$ (the opposite case is similar). Using Theorem 4.2 , we obtain $\dot{\hat{\theta}}^{\circ}(t)=\sqrt{2}\left(\mathbb{E}-\mathcal{U}\left(\hat{\theta}^{\circ}(t)\right)\right)^{1 / 2}>0$ for $t \in\left(t_{+} ; t_{+}+\tau\right)$. Hence

$$
\frac{\dot{\hat{\theta}}^{\circ}(t)}{\sqrt{\mathbb{E}-\mathcal{U}\left(\hat{\theta}^{\circ}(t)\right)}}=\sqrt{2} \quad \text { for } \quad t \in\left(t_{+} ; t_{+}+\tau\right)
$$

and

$$
\int_{t_{+}}^{t} \frac{\dot{\hat{\theta}}^{\circ}(s) \mathrm{d} s}{\sqrt{\mathbb{E}-\mathcal{U}\left(\hat{\theta}^{\circ}(s)\right)}}=\sqrt{2}\left(t-t_{+}\right) \quad \text { for } \quad t \in\left(t_{+} ; t_{+}+\tau\right] .
$$

The change of variables $\mathrm{d} \theta^{\circ}=\dot{\hat{\theta}}^{\circ}(s) \mathrm{d} s, \theta^{\circ}=\hat{\theta}^{\circ}(s)$ is admissible, since the function $\hat{\theta}^{\circ} \in W_{\infty}^{2}$ is strictly monotone increasing for $t \in\left[t_{+} ; t_{+}+\tau\right]$. This change proves both $\left(\mathbb{E}-\mathcal{U}\left(\theta^{\circ}\right)\right)^{-1 / 2} \in L_{1}\left(\theta_{0}^{\circ}, \theta_{0}^{\circ}+\varepsilon\right)$ for some $\varepsilon>0$ and $(4.5)$ for any solution $\hat{\theta}^{\circ}$ with $t_{+}<\infty$.

Let us now prove item 2 of the theorem. Suppose that $\left(\mathbb{E}-\mathcal{U}\left(\theta^{\circ}\right)\right)^{-1 / 2} \in L_{1}\left(\theta_{0}^{\circ}, \theta_{0}^{\circ}+\varepsilon\right)$ for some $\varepsilon>0$ (the case $\varepsilon<0$ is similar). Then $(r)$ or $(b)$ is fulfilled, and the function

$$
G\left(\theta^{\circ}\right) \stackrel{\text { def }}{=} \int_{\theta_{0}^{\circ}}^{\theta^{\circ}} \frac{\mathrm{d} \psi}{\sqrt{\mathbb{E}-\mathcal{U}(\psi)}}
$$

is strictly increasing for $\theta^{\circ} \in\left[\theta_{0}^{\circ} ; \theta_{r}^{\circ}\right]$. Moreover, $G\left(\theta_{0}^{\circ}\right)=0$ and $G\left(\theta_{r}^{\circ}\right) \geq \sqrt{2} \tau$, since $\mathbb{E}-\mathcal{U}\left(\theta^{\circ}\right) \leq \frac{1}{2} M^{2}$. Hence for any $t \in[0 ; \tau]$, there exists $\tilde{\theta}^{\circ}(t)$ such that $G\left(\tilde{\theta}^{\circ}(t)\right)=\sqrt{2} t$. Moreover, since $G \in C^{1}\left(\theta_{0}^{\circ}, \theta_{r}^{\circ}\right)$ and $G^{\prime}\left(\theta^{\circ}\right) \neq 0$ for 
$\theta^{\circ} \in\left(\theta_{0}^{\circ}, \theta_{r}^{\circ}\right)$, we obtain $\tilde{\theta}^{\circ} \in C^{1}(0 ; \tau)$ and $\dot{\tilde{\theta}}^{\circ}=\sqrt{2}\left(\mathbb{E}-\mathcal{U}\left(\tilde{\theta}^{\circ}\right)\right)^{1 / 2}>0$ by the inverse function theorem. Hence, $\tilde{\theta}^{\circ}$ is a solution to (4.1) by Theorem 4.2 .

Since $\tilde{\theta}^{\circ}(0)=\theta_{0}^{\circ}$ and $\dot{\tilde{\theta}}^{\circ}(0)=0$, it remains to put

$$
\hat{\theta}^{\circ}(t)= \begin{cases}\theta_{0}^{\circ} & \text { for } t \in\left[0 ; t_{+}\right] \\ \tilde{\theta}\left(t-t_{+}\right) & \text {for } t \in\left(t_{+} ; t_{+}+\tau\right] .\end{cases}
$$

Indeed, the function $\hat{\theta}$ is $C^{1}$ and its second derivative is $L_{\infty}$ and satisfies (4.1) for a.e. $t$.

So, uniqueness may fail only if a solution has zero speed $\theta_{1}^{\circ}=0$ at a point $\theta_{0}^{\circ}$ and $0 \in \mathcal{U}^{\prime}\left(\theta_{0}^{\circ}\right)$. Moreover, if $\partial \Omega^{\circ}$ is $W_{\infty}^{2}$ in a neighborhood of the point $\left(\cos _{\Omega^{\circ}} \theta_{0}^{\circ}, \sin _{\Omega^{\circ}} \theta_{0}^{\circ}\right)$ then uniqueness persists, since improper integral (4.5) is not finite (or by Prop. 4.3). Let us develop some easy to check condition that guarantees absence of uniqueness.

Proposition 4.7. If $\mathcal{U}^{\prime}\left(\theta_{0}^{\circ}+0\right)<0$, then $\left(\mathbb{E}-\mathcal{U}\left(\theta^{\circ}\right)\right)^{-1 / 2} \in L_{1}\left(\theta_{0}^{\circ}, \theta_{0}^{\circ}+\varepsilon\right)$ for $\mathbb{E}=\mathcal{U}\left(\theta_{0}^{\circ}\right)$ and some $\varepsilon>0$. A similar result holds for the case $\mathcal{U}^{\prime}\left(\theta_{0}^{\circ}-0\right)>0$.

Proof. Indeed, in this case, $\mathcal{U}\left(\theta^{\circ}\right) \leq \mathbb{E}-\frac{1}{2} \delta\left(\theta^{\circ}-\theta_{0}^{\circ}\right)$ for $\theta^{\circ}$ in a right neighborhood of $\theta_{0}^{\circ}$.

Let us give a geometric explanation of the pair of conditions $0 \in \mathcal{U}^{\prime}\left(\theta_{0}^{\circ}\right)$ and $\mathcal{U}^{\prime}\left(\theta_{0}^{\circ}+0\right)<0$ (which together guarantee absence of uniqueness for solutions with $\left.\theta_{1}^{\circ}=0\right)$. Denote $Q=\left(\cos _{\Omega^{\circ}} \theta^{\circ}, \sin _{\Omega^{\circ}} \theta^{\circ}\right) \in \partial \Omega^{\circ}$. Obviously, if $d f(Q)=0$, then $\mathcal{U}^{\prime}\left(\theta_{0}^{\circ}\right)=\{0\}$. So we assume that $d f(Q) \neq 0$. In this case, $d f(Q)$ defines an (affine) hyperplane $\Pi=\left\{(p, q) \in \mathbb{R}^{2 *}:\langle(p, q), d f(Q)\rangle \leq\langle Q, d f(Q)\rangle\right\}$. The line $\partial \Pi$ is tangent to $\partial \Omega^{\circ}$ at $Q$ iff $0 \in \mathcal{U}^{\prime}\left(\theta_{0}^{\circ}\right)$. The set of all $\theta \leftrightarrow \theta_{0}^{\circ}$ has the form $\left[\theta_{-} ; \theta_{+}\right]+2 \mathbb{S} \mathbb{Z}$, and the counterclockwise tangent ray at $Q$ to $\partial \Omega^{\circ}$ is defined by $\theta_{+}$: this ray is co-directed with $\left(-\sin _{\Omega} \theta_{+}, \cos _{\Omega} \theta_{+}\right)$. In these notations, $\mathcal{U}^{\prime}\left(\theta_{0}^{\circ}+0\right)=\left\langle d f(Q),\left(-\sin _{\Omega} \theta_{+}, \cos _{\Omega} \theta_{+}\right)\right\rangle<0$. Therefore, the ray belongs to the interior of the supporting hyperplane $\Pi$ at $Q$ to $\Omega^{\circ}$, defined by $d f(Q) \neq 0$.

\subsection{Admissible controls}

In what follows, solutions to (4.1) often determine a lift of extremals into the cotangent bundle, and $\hat{\theta}(t) \leftrightarrow$ $\hat{\theta}^{\circ}(t)$ determines control on extremals. So the last thing we want to develop in this section is to find all functions $\hat{\theta}(t)$ such that, for a.e. $t, \hat{\theta}(t) \leftrightarrow \hat{\theta}^{\circ}(t)$ and

$$
\ddot{\hat{\theta}}^{\circ}=\mathrm{d} f_{p} \sin _{\Omega} \hat{\theta}(t)-\mathrm{d} f_{q} \cos _{\Omega} \hat{\theta}(t)
$$

(measurable functions $\hat{\theta}(t)$ satisfying these two properties for a.e. $t$ will be called admissible).

First, suppose that $\theta_{1}^{\circ} \neq 0$. In this case, there exists a unique solution $\hat{\theta}^{\circ}(t)$ to (4.1), (4.2) for $t$ in a neighborhood of $t=0$ by Proposition 4.4. Then $\dot{\hat{\theta}}^{\circ}(t) \neq 0$ in a neighborhood of $t=0$. Since for a.e. $\theta^{\circ}$ there exists a unique $\theta \leftrightarrow \theta^{\circ}$, we obtain that there exists a unique $\hat{\theta}(t) \leftrightarrow \hat{\theta}^{\circ}(t)$ for a.e. $t$ in a neighborhood of $t=0$. Hence there exists a unique (up to a set of zero measure) admissible $\hat{\theta}(t)$.

Now suppose that $\theta_{1}^{\circ}=0$. In this case, we want to examine, whether $\hat{\theta}^{\circ}(t) \equiv \theta_{0}^{\circ}$ is a solution, and if it is, we want to find an admissible $\hat{\theta}(t)$. Obviously,

$$
-\mathrm{d} f_{p}\left(Q_{0}\right) \sin _{\Omega} \hat{\theta}(t)+\mathrm{d} f_{q}\left(Q_{0}\right) \cos _{\Omega} \hat{\theta}(t)=-\ddot{\hat{\theta}}^{\circ}(t)=0,
$$

where we denote $Q_{0}=\left(\cos _{\Omega^{\circ}} \theta_{0}^{\circ}, \sin _{\Omega^{\circ}} \theta_{0}^{\circ}\right)$ for short. Since $\hat{\theta}(t) \leftrightarrow \theta_{0}^{\circ}$,

$$
\cos _{\Omega^{\circ}} \theta_{0}^{\circ} \cos _{\Omega} \hat{\theta}(t)+\sin _{\Omega^{\circ}} \theta_{0}^{\circ} \sin _{\Omega} \hat{\theta}(t)=1 .
$$


Hence, $x=\cos _{\Omega} \hat{\theta}(t)$ and $y=\sin _{\Omega} \hat{\theta}(t)$ satisfy the following system of two linear equations:

$$
\left\{\begin{array}{l}
x \cos \Omega^{\circ} \theta^{\circ}+y \sin _{\Omega^{\circ}} \theta^{\circ}=1 ; \\
x f_{q}(Q(t))-y f_{p}(Q(t))=0 ;
\end{array} \Leftrightarrow\left(\begin{array}{cc}
\cos \Omega^{\circ} \theta^{\circ} & \sin _{\Omega^{\circ}} \theta^{\circ} \\
f_{q}(Q(t)) & -f_{p}(Q(t))
\end{array}\right)\left(\begin{array}{l}
x \\
y
\end{array}\right)=\left(\begin{array}{l}
1 \\
0
\end{array}\right) .\right.
$$

(1) If $d f\left(Q_{0}\right) \not \perp Q_{0}$, then the system has a unique solution $\left(x_{0}, y_{0}\right)$, since the matrix in the left-hand side has non-zero determinant. Moreover, the point $\left(x_{0}, y_{0}\right)$ belongs to the supporting line to $\Omega$ determined by the first equation of the system. So, if $d f\left(Q_{0}\right) \not \perp Q_{0}$, then there are two possibilities.

(a) $\left(x_{0}, y_{0}\right) \in \partial \Omega$ iff $0 \in \mathcal{U}^{\prime}\left(\theta_{0}^{\circ}\right)$. In this case, $\hat{\theta}^{\circ}(t) \equiv \theta_{0}^{\circ}$ is a solution to (4.1). Moreover, there exists a unique (up to a set of zero measure) admissible $\hat{\theta}(t) \equiv \theta_{0}$ where $x_{0}=\cos _{\Omega} \theta_{0}$ and $y_{0}=\sin _{\Omega} \theta_{0}$. This case usually corresponds to singular extremals.

(b) $\left(x_{0}, y_{0}\right) \notin \partial \Omega$ iff $0 \notin \mathcal{U}^{\prime}\left(\theta_{0}^{\circ}\right)$. In this case, $\hat{\theta}^{\circ}(t) \equiv \theta_{0}^{\circ}$ is not a solution to (4.1)(1a). By Proposition 4.5 there exists a unique solution $\hat{\theta}^{\circ}(t)$ to $(4.1),(4.2)$, and $\dot{\hat{\theta}}^{\circ}(t) \neq 0$ in a punctured neighborhood of $t=0$. Hence the admissible function $\hat{\theta}(t)$ is unique (again up to a zero measure) in this neighborhood.

(2) If $d f\left(Q_{0}\right) \perp Q_{0}$, then $\mathcal{U}^{\prime}\left(\theta_{0}^{\circ}\right)$ is a singleton. Indeed, $\left(f_{p}\left(Q_{0}\right), f_{q}\left(Q_{0}\right)\right)=\lambda\left(-\sin _{\Omega^{\circ}} \theta_{0}^{\circ}, \cos \Omega_{\Omega^{\circ}} \theta_{0}^{\circ}\right)$ for some $\lambda \in$ $\mathbb{R}$ and $\mathcal{U}^{\prime}\left(Q_{0}\right)=\left\{-f_{p}\left(Q_{0}\right) \sin _{\Omega} \theta+f_{q}\left(Q_{0}\right) \cos _{\Omega} \theta\right.$ for all $\left.\theta \leftrightarrow \theta_{0}^{\circ}\right\}$. Hence by the generalized Pythagorean identity (see Sect. 2.2), we have $\mathcal{U}^{\prime}\left(\theta_{0}^{\circ}\right)=\{\lambda\}$. There are two possibilities.

(a) If $d f\left(Q_{0}\right) \neq 0$, then $\mathcal{U}^{\prime}\left(\theta_{0}^{\circ}\right) \neq 0$ and $\hat{\theta}^{\circ}(t) \equiv \theta_{0}^{\circ}$ is not a solution to (4.1).

(b) If $d f\left(Q_{0}\right)=0$, then $\mathcal{U}^{\prime}\left(\theta_{0}^{\circ}\right)=0$ and $\hat{\theta}^{\circ}(t) \equiv \theta_{0}^{\circ}$ is a solution to (4.1). In this case, any measurable function $\hat{\theta}(t)$ satisfying $\hat{\theta}(t) \leftrightarrow \theta_{0}^{\circ}$ is admissible. If $\Omega^{\circ}$ has a corner at $Q_{0}$, then $\hat{\theta}(t)$ is not unique and vice versa. This case also corresponds to singular extremals.

Remark 4.8. To distinguish cases (1a) and (2b) we use the following terms. In case (1a), we say that $\hat{\theta}(t)$ is general singular; and in case $(2 \mathrm{~b})$, we say that $\hat{\theta}(t)$ is special singular.

\section{LEFT-INVARIANT SUB-FINSLER PROBLEMS ON 3D UNIMODULAR LIE GROUPS}

\subsection{Problem statement and normalization}

Let $G$ be a 3-dimensional unimodular Lie group, and $L=T_{\mathrm{Id}} G$ its Lie algebra, where Id is the identity element of $G$. Thus the following cases are possible [28]:

- $L=\mathfrak{h}_{3}$ is the Heisenberg algebra,

- $L=\mathfrak{s u _ { 2 }}$ is the Lie algebra of the group of $2 \times 2$ unitary matrices,

- $L=\mathfrak{s l}_{2}$ is the Lie algebra of the group of $2 \times 2$ real unimodular matrices,

- $L=\mathfrak{s e}_{2}\left(L=\mathfrak{s h}_{2}\right)$ is the Lie algebra of the group of Euclidean (resp. hyperbolic) motions of the 2dimensional plane.

Let $\Delta \subset L$ be a 2-dimensional subspace that is not a subalgebra, and let $U \subset \Delta$ be a convex compact set such that $0 \in \operatorname{int}_{\Delta} U$. We consider the following left-invariant time-optimal problem:

$$
\begin{array}{ll}
\dot{q} \in q U, & q \in G, \\
q(0)=q_{0}, & q(T)=q_{1}, \\
T \rightarrow \min . &
\end{array}
$$

Remark 5.1. If $U$ is an ellipse centered at the origin, then problem (5.1)-(5.3) is sub-Riemannian. Some cases of this problem were studied in papers [11-13, 18-20, 34, 41, 42].

Remark 5.2. Problem (5.1)-(5.3) defines a distance function on the group in a classical way. If $U=-U$, then the distance is symmetric. In this case problem (5.1)-(5.3) is usually called sub-Finsler in literature. If $U \neq-U$ 
then the distance is not symmetric, but it defines a regular Hausdorff topology on the group. So we will call such a problem as well sub-Finsler for short.

Since problem (5.1)-(5.3) is left-invariant, we can assume that $q_{0}=\mathrm{Id}$.

By the theorem of Agrachev-Barilari [2] on classification of contact left-invariant sub-Riemannian structures on $3 \mathrm{D}$ unimodular Lie groups, there exists a basis $L=\operatorname{span}\left(X_{1}, X_{2}, X_{3}\right)$ such that

$$
\begin{aligned}
& \Delta=\operatorname{span}\left(X_{1}, X_{2}\right), \\
& {\left[X_{1}, X_{2}\right]=X_{3}, \quad\left[X_{3}, X_{1}\right]=(\chi+\kappa) X_{2}, \quad\left[X_{3}, X_{2}\right]=(\chi-\kappa) X_{1},} \\
& \chi=\kappa=0 \quad \text { or } \quad\left(\chi^{2}+\kappa^{2}=1, \chi \geq 0\right) .
\end{aligned}
$$

The case $\chi=\kappa=0$ corresponds to the Heisenberg algebra $L=\mathfrak{h}_{3}$, and the case when $G$ is the Heisenberg group was first studied in [17], where curves having closed projection on the plane $\left(x_{1}, x_{2}\right)$ were found without using Pontryagin maximum principle. A full description of all geodesics in this problems was obtained in [10] with the help of Pontryagin maximum principle, and in [31] with the help of convex trigonometry. Thus we assume in the sequel that $\chi^{2}+\kappa^{2}=1, \chi \geq 0$.

The two-parameter group of transformations $\left(X_{1}, X_{2}, X_{3}\right) \mapsto\left(\alpha X_{1}, \beta X_{2}, \alpha \beta X_{3}\right), \alpha, \beta>0$, reduces product table (5.4) to the following one:

$$
\begin{aligned}
& {\left[X_{1}, X_{2}\right]=X_{3}, \quad\left[X_{3}, X_{1}\right]=a X_{2}, \quad\left[X_{3}, X_{2}\right]=b X_{1},} \\
& a, b \in\{ \pm 1,0\}, \quad a+b \geq 0, \quad(a, b) \neq(0,0),
\end{aligned}
$$

where $a=\operatorname{sgn}(\chi+\kappa), b=\operatorname{sgn}(\chi-\kappa)$. So we get the following table of reduction from parameters $(\chi, \kappa)$ to parameters $(a, b)$ :

$$
\begin{aligned}
& -\chi+\kappa<0, \chi-\kappa>0\left(L=\mathfrak{s l}_{2}\right) \quad \Rightarrow \quad a=-1, b=1, \\
& -\chi+\kappa=0, \chi-\kappa>0\left(L=\mathfrak{s h}_{2}\right) \Rightarrow a=0, b=1 \text {, } \\
& -\chi+\kappa>0, \chi-\kappa>0\left(L=\mathfrak{s l}_{2}\right) \quad \Rightarrow \quad a=1, b=1 \text {, } \\
& -\chi+\kappa>0, \chi-\kappa=0\left(L=\mathfrak{s e}_{2}\right) \quad \Rightarrow \quad a=1, b=0, \\
& -\chi+\kappa>0, \chi-\kappa<0\left(L=\mathfrak{s u}_{2}\right) \quad \Rightarrow \quad a=1, b=-1 \text {. }
\end{aligned}
$$

Parametrize the set $U \subset \Delta$ by a control parameter $u=\left(u_{1}, u_{2}\right) \in \Omega \subset \mathbb{R}^{2}$, so that $U=\left\{u_{1} X_{1}+u_{2} X_{2} \mid u=\right.$ $\left.\left(u_{1}, u_{2}\right) \in \Omega\right\}$. Then problem $(5.1)-(5.3)$ reads as follows:

$$
\begin{aligned}
& \dot{q}=u_{1} X_{1}(q)+u_{2} X_{2}(q), \quad q \in G, \quad u=\left(u_{1}, u_{2}\right) \in \Omega, \\
& q(0)=q_{0}=\mathrm{Id}, \quad q(T)=q_{1}, \\
& T \rightarrow \min
\end{aligned}
$$

where left-invariant vector fields $X_{1}, X_{2}$ on the Lie group $G$ satisfy conditions (5.5), (5.6).

\subsection{Pontryagin maximum principle}

We describe extremals of problem (5.7)-(5.9). Notice that optimal controls in problem (5.7)-(5.9) exist by the Rashevsky-Chow and Filippov theorems [4]. Denote the cotangent bundle of the Lie group $G$ as $T^{*} G$, and its points as $\lambda \in T^{*} G$. Introduce linear on fibers of $T^{*} G$ Hamiltonians corresponding to the basis vector fields: $h_{i}(\lambda)=\left\langle\lambda, X_{i}(q)\right\rangle, q=\pi(\lambda), i=1,2,3$, where $\pi: T^{*} G \rightarrow G$ is the canonical projection.

Apply the Pontryagin maximum principle $[4,37]$ to problem $(5.7)-(5.9)$. The Hamiltonian function of PMP is $\mathcal{H}(u, \lambda)=u_{1} h_{1}(\lambda)+u_{2} h_{2}(\lambda)$. The Pontryagin maximum principle states that if a curve $q(t)$ and a control $u(t), t \in[0, T]$, are optimal, then there exists a Lipschitzian curve $\lambda_{t} \in T_{q(t)}^{*} G, \lambda_{t} \neq 0, t \in[0, T]$, that satisfies the following conditions: 
- the maximality condition

$$
u_{1} h_{1}+u_{2} h_{2}=\max _{w \in \Omega}\left(w_{1} h_{1}+w_{2} h_{2}\right)=s_{\Omega}\left(h_{1}, h_{2}\right)=: H
$$

- the Hamiltonian system

$$
\left\{\begin{array}{l}
\dot{h}_{1}=-u_{2} h_{3}, \\
\dot{h}_{2}=u_{1} h_{3}, \\
\dot{h}_{3}=-a u_{1} h_{2}-b u_{2} h_{1}, \\
\dot{q}=u_{1} X_{1}+u_{2} X_{2},
\end{array}\right.
$$

- and the identity $H \equiv$ const $\geq 0$ along any trajectory.

Abnormal case. Let $H \equiv 0$. Then $h_{1}=h_{2} \equiv 0$, and since $\lambda_{t} \neq 0$ then $h_{3} \neq 0$. Then the first two equations of system (5.11) yield $u_{2} h_{3}=u_{1} h_{3} \equiv 0$, thus $u_{1}=u_{2} \equiv 0$. So abnormal trajectories are constant, $q \equiv$ const.

Cauchy nonuniqueness of extremals. Extremals are trajectories of a nonautonomous ODE - the Hamiltonian system of PMP (5.11). So there can be two different extremals $\lambda_{t}^{1} \not \equiv \lambda_{t}^{2}$ that intersect one another: $\lambda_{t_{0}}^{1}=\lambda_{t_{0}}^{2}$. We call such phenomenon Cauchy nonuniqueness of extremals. There can be two reasons for Cauchy nonuniqueness:

- Cauchy nonuniqueness of extremals due to different controls: the point $\lambda_{t_{0}}$ of an extremal $\lambda_{t}$ determines a nonunique control $u\left(t_{0}\right)$ via the maximality condition of PMP (5.10). Then we have different controls $u_{1}(t) \not \equiv u_{2}(t)$ and the corresponding different extremals $\lambda_{t}^{1} \not \equiv \lambda_{t}^{2}$ with $\lambda_{t_{0}}^{1}=\lambda_{t_{0}}^{2}=\lambda_{t_{0}}$.

- Cauchy nonuniqueness due to nonsmoothness: the Hamiltonian vector field $\vec{H}$ corresponding to maximized Hamiltonian $H$ of PMP is not $C^{1}$-smooth. Then, even if maximality condition (5.10) of PMP defines uniquely control as a function of covector $\lambda \mapsto u(\lambda)$, the resulting vector field $\vec{H}(\lambda)$ may have different trajectories with the same initial point (like the ODE $\dot{x}=x^{1 / 3}$ ).

In order to distinguish different reasons for Cauchy nonuniqueness of extremals, introduce the following sets:

$$
\mathfrak{S}_{k}=\left\{\left(h_{1}, h_{2}\right) \in \mathbb{R}^{2} \mid H \notin C^{k}\left(h_{1}, h_{2}\right)\right\}, \quad k=1,2 .
$$

It is obvious that $\{0\} \subset \mathfrak{S}_{1} \subset \mathfrak{S}_{2}$ and that $\mathfrak{S}_{1}$ consists of a finite or countable set of rays beginning at the origin. Moreover, the set of points where $\partial \Omega^{\circ}$ is not $C^{2}$ has zero measure by Alexandrov theorem [5]. Hence the set $\mathfrak{S}_{2}$ consists of rays beginning at the origin, and intersection of $\mathfrak{S}_{2}$ with the unit circle has zero measure on it.

If $\left(h_{1}, h_{2}\right) \in \mathfrak{S}_{1}$, then maximality condition (5.10) determines a nonunique control, and we may have classical singular trajectories. Moreover, bang-bang extremals can join singular extremals, so mixed extremals (concatenations of bang-bang and singular extremals) may appear because of Cauchy nonuniqueness due to different controls.

If $\left(h_{1}, h_{2}\right) \in \mathfrak{S}_{2} \backslash \mathfrak{S}_{1}$, then $H \in C^{1}$ and maximality condition (5.10) determines a unique control $u=\nabla H$. Although, the Hamiltonian vector field $\vec{H} \in C^{0} \backslash C^{1}$, thus we may have Cauchy nonuniqueness of extremals due to nonsmoothness, and this case needs additional accuracy.

Finally, if $\left(h_{1}, h_{2}\right) \in \mathbb{R}^{2} \backslash \mathfrak{S}_{2}$, then $H \in C^{2}$, thus $\vec{H} \in C^{1}$, and there is no Cauchy nonuniqueness of extremals.

Normal case. Let $H>0$. The maximized Hamiltonian $H\left(h_{1}, h_{2}\right)$ is a convex positively homogeneous of order one function in the plane $\mathbb{R}_{h_{1}, h_{2}}^{2}$. Maximality condition (5.10) yields $u=\left(u_{1}, u_{2}\right) \in \partial \Omega$, thus

$$
u_{1}=\cos _{\Omega} \theta, \quad u_{2}=\sin _{\Omega} \theta
$$


for an angle $\theta \in \mathbb{R} /(2 \mathbb{S} \mathbb{Z})$. Moreover, condition (5.10) yields $\left(h_{1}, h_{2}\right) \in H \partial \Omega^{\circ}$, thus

$$
h_{1}=H \cos \Omega^{\circ} \theta^{\circ}, \quad h_{2}=H \sin _{\Omega^{\circ}} \theta^{\circ}
$$

for an angle $\theta^{\circ} \in \mathbb{R} /\left(2 \mathbb{S}^{\circ} \mathbb{Z}\right), \mathbb{S}^{\circ}=\mathbb{S}\left(\Omega^{\circ}\right)$. Since

$$
\cos _{\Omega} \theta \cos \Omega^{\circ} \theta^{\circ}+\sin _{\Omega} \theta \sin _{\Omega^{\circ}} \theta^{\circ}=\frac{u_{1} h_{1}+u_{2} h_{2}}{H}=1,
$$

then $\theta \leftrightarrow \theta^{\circ}$ for a.e. $t$. Further, we have

$$
\dot{\theta^{\circ}}=\frac{h_{1} \dot{h}_{2}-h_{2} \dot{h}_{1}}{H^{2}}=\left(H \cos _{\Omega^{\circ}} \theta^{\circ} \cos _{\Omega} \theta h_{3}+H \sin _{\Omega^{\circ}} \theta^{\circ} \sin _{\Omega} \theta h_{3}\right) / H^{2}=h_{3} / H .
$$

So the vertical subsystem of Hamiltonian system (5.11) reduces to the system

$$
\left\{\begin{array}{l}
\dot{\theta^{\circ}}=h_{3} / H, \\
\dot{h}_{3}=-H\left(a \cos _{\Omega} \theta \sin _{\Omega^{\circ}} \theta^{\circ}+b \sin _{\Omega} \theta \cos _{\Omega^{\circ}} \theta^{\circ}\right) .
\end{array}\right.
$$

Using Theorem 4.2, we see that $\frac{1}{2}{\dot{\theta^{\circ}}}^{2}+\frac{1}{2}\left(a \sin _{\Omega^{\circ}}^{2} \theta^{\circ}-b \cos _{\Omega^{\circ}}^{2} \theta^{\circ}\right)$ is a first integral of the system. This fact can also be obtained via the left-invariant structure of the system. Indeed, the function $\mathcal{C}=\frac{1}{2}\left(h_{3}^{2}-b h_{1}^{2}+a h_{2}^{2}\right)$ is a Casimir on the Lie coalgebra $L^{*}$ for Lie-Poisson bracket, thus the ratio $\mathbb{E}=\mathcal{C} / H$ is a first integral of system (5.14). Decompose

$$
\begin{aligned}
& \mathbb{E}=\frac{1}{2 H} h_{3}^{2}+\mathcal{U}\left(\theta^{\circ}\right), \\
& \mathcal{U}\left(\theta^{\circ}\right)=\frac{1}{2 H}\left(a h_{2}^{2}-b h_{1}^{2}\right)=\frac{H}{2}\left(a \sin _{\Omega^{\circ}}^{2} \theta^{\circ}-b \cos _{\Omega^{\circ}}^{2} \theta^{\circ}\right) .
\end{aligned}
$$

We call $\frac{1}{2 H} h_{3}^{2}$ the kinetic energy, $\mathcal{U}\left(\theta^{\circ}\right)$ the potential energy, and $\mathbb{E}$ the full energy of system (5.14). This system turns out to be a Hamiltonian system with the Hamiltonian $\mathbb{E}$ :

$$
\begin{cases}\dot{\theta^{\circ}}=h_{3} / H, & \theta^{\circ} \in \mathbb{R} /\left(2 \mathbb{S}^{\circ} \mathbb{Z}\right), \\ \dot{h}_{3} \in-\mathcal{U}^{\prime}\left(\theta^{\circ}\right), & h_{3} \in \mathbb{R} .\end{cases}
$$

If $\Omega^{\circ}$ has $C^{2}$-smooth boundary, then $\cos \Omega^{\circ} \theta^{\circ}$ and $\sin _{\Omega^{\circ}} \theta^{\circ}$ are $C^{2}$-smooth functions by Proposition 2.5. In this case we have a classical smooth Hamiltonian system. If $\partial \Omega^{\circ}$ has corners then $\mathcal{U}^{\prime}\left(\theta^{\circ}\right)$ is an interval between left and right derivatives at a corner $\theta^{\circ}$. In this case we read Hamiltonian system (5.15) in the Filippov sense [26], and it may have multiple solutions with the same initial data.

Remark 5.3. The function $\mathcal{U}\left(\theta^{\circ}\right)$ is Lipschitzian in general, since $\partial \Omega^{\circ}$ is Lipschitzian, and system (5.15) may have non-unique solution. But if an open interval on $\partial \Omega^{\circ}$ is $C^{2}$, then $\mathcal{U} \in C^{2}$ for corresponding angles $\theta^{\circ}$, and system (5.15) has a unique solution for any given initial data. In other words, uniqueness may fail only on the set $\mathfrak{S}_{2}$.

The phase portrait of system (5.15) is completely determined by the potential energy $\mathcal{U}\left(\theta^{\circ}\right)$.

We call an extremal arc $\lambda_{t}, t \in[\alpha, \beta], \alpha<\beta$ :

- a bang arc if $\left(h_{1}, h_{2}\right)\left(\lambda_{t}\right) \notin \mathfrak{S}_{2}$ for all $t \in(\alpha, \beta)$,

- a bang-bang arc if $\left(h_{1}, h_{2}\right)\left(\lambda_{t}\right) \notin \mathfrak{S}_{2}$ for a.e. $t \in(\alpha, \beta)$,

- a singular arc if $\left(h_{1}, h_{2}\right)\left(\lambda_{t}\right) \in \mathfrak{S}_{2}$ for all $t \in[\alpha, \beta]$, 
- mixed if it is a concatenation of a finite number of bang-bang and singular arcs.

Remark 5.4. Usually, the term "singular extremal" is used for extremals where PMP defines a non-unique control. On these extremals uniqueness of solutions to PMP is lost. Moreover, singular extremals were studied mostly for problems with one-dimensional control, where analogues of the sets $\mathfrak{S}_{1}$ and $\mathfrak{S}_{2}$ coincide one with another. We believe that the term "non-singular extremals" must be applied to extremals where everything is regular, i.e., PMP has the property of uniqueness of solutions. Therefore we decided to use term "singular" in this paper for extremals in $\mathfrak{S}_{2}$.

In examples considered below we prove that Fuller's phenomenon is not present here, i.e., along any extremal arc duration of all maximal bang arcs is separated from zero, thus any extremal arc is either bang-bang, singular, or mixed.

\subsection{Bang-bang extremals}

System (5.15) has fixed points $\left(\theta^{\circ}, h_{3}\right)=\left(\theta_{*}^{\circ}, 0\right)$, where $\mathcal{U}^{\prime}\left(\theta_{*}^{\circ}\right) \ni 0$.

If $\mathcal{U}\left(\theta^{\circ}\right)$ has a local minimum at $\theta_{*}^{\circ}$, decreases at an interval $\left(\theta_{*}^{\circ}-\varepsilon, \theta_{*}^{\circ}\right]$ and increases at an interval $\left[\theta_{*}^{\circ}, \theta_{*}^{\circ}+\varepsilon\right)$, then the phase portrait of system (5.15) has a fixed point $\left(\theta^{\circ}, h_{3}\right)=\left(\theta_{*}^{\circ}, 0\right)$ of the center type.

If $\mathcal{U}\left(\theta^{\circ}\right)$ has a local maximum at $\theta_{*}^{\circ}$, increases at an interval $\left(\theta_{*}^{\circ}-\varepsilon, \theta_{*}^{\circ}\right]$ and decreases at an interval $\left[\theta_{*}^{\circ}, \theta_{*}^{\circ}+\varepsilon\right)$, then the phase portrait of system (5.15) has a fixed point $\left(\theta^{\circ}, h_{3}\right)=\left(\theta_{*}^{\circ}, 0\right)$ of the saddle type. The separatrix entering the fixed point $\left(\theta_{*}^{\circ}, 0\right)$ in the strip $\theta^{\circ} \in\left(\theta_{*}^{\circ}-\varepsilon, \theta_{*}^{\circ}\right)$ comes to the fixed point for the time evaluated by the improper integral (see Section 4.3)

$$
I=H \int_{\theta_{*}^{\circ}-\varepsilon}^{\theta_{*}^{\circ}} \frac{\mathrm{d} \theta^{\circ}}{\sqrt{2\left(\mathbb{E}-\mathcal{U}\left(\theta^{\circ}\right)\right)}} .
$$

This time is finite or infinite depending on whether the integral $I$ converges or not. Similarly for the separatrix in the strip $\theta^{\circ} \in\left(\theta_{*}^{\circ}, \theta_{*}^{\circ}+\varepsilon\right)$. So, unlike the classic case, separatrix can enter the fixed point of saddle type in finite time. In this case, we have Cauchy nonuniqueness of extremals.

\subsection{Singular extremals}

Since the singular set $\mathfrak{S}_{2}$ of Hamiltonian $H$ consists of rays starting at the origin forming set of zero measure, and $s_{\Omega}\left(h_{1}, h_{2}\right)=H=$ const, any singular $\operatorname{arc} \lambda_{t}$ satisfies the identities

$$
h_{1}\left(\lambda_{t}\right) \equiv \text { const }, \quad h_{2}\left(\lambda_{t}\right) \equiv \text { const. }
$$

If the corresponding extremal trajectory $q(t)$ is not constant, then the Hamiltonian system (5.11) implies additionally that

$$
\begin{aligned}
& h_{3}\left(\lambda_{t}\right) \equiv 0, \\
& a u_{1}(t) h_{2}\left(\lambda_{t}\right)+b u_{2}(t) h_{1}\left(\lambda_{t}\right) \equiv 0 .
\end{aligned}
$$

Taking into account (5.12), (5.13) and the maximality condition (5.10), we conclude that a singular extremal satisfies the following conditions:

$$
\begin{aligned}
& \theta^{\circ} \equiv \text { const, } \\
& h_{3} \equiv 0, \\
& a \sin _{\Omega^{\circ}} \theta^{\circ} \cos _{\Omega} \theta+b \cos _{\Omega^{\circ}} \theta^{\circ} \sin _{\Omega} \theta \equiv 0, \\
& \cos _{\Omega^{\circ}} \theta^{\circ} \cos _{\Omega} \theta+\sin _{\Omega^{\circ}} \theta^{\circ} \sin _{\Omega} \theta \equiv 1,
\end{aligned}
$$




$$
\left(h_{1}\left(\lambda_{t}\right), h_{2}\left(\lambda_{t}\right)\right) \in \mathfrak{S}_{2} .
$$

Conversely, any arc $\lambda_{t}$ and control $u(t)$ that satisfy conditions (5.20)-(5.24) are singular.

Hence $\theta^{\circ}=$ const is a clue parameter determining the singular extremal.

Let us investigate solutions of system (5.20)-(5.24) (the investigation is similar to Sect. 4.4). We start with equations $(5.22),(5.23)$. They may be written in the following form:

$$
A\left(\begin{array}{l}
u_{1} \\
u_{2}
\end{array}\right)=\left(\begin{array}{l}
1 \\
0
\end{array}\right)
$$

where $u=\left(u_{1}, u_{2}\right)=\left(\cos _{\Omega} \theta, \sin _{\Omega} \theta\right)$ and

$$
A=\left(\begin{array}{cc}
\cos _{\Omega^{\circ}} \theta^{\circ} & \sin _{\Omega^{\circ}} \theta^{\circ} \\
a \sin _{\Omega^{\circ}} \theta^{\circ} & b \cos _{\Omega^{\circ}} \theta^{\circ}
\end{array}\right)
$$

Any solution to linear system (5.25) must belong to the support line of $\Omega$ defined by the first equation $\cos _{\Omega^{\circ}} \theta^{\circ} u_{1}+\sin _{\Omega^{\circ}} \theta^{\circ} u_{2}=1$, but it may happen that there exist solutions to linear system (5.25) that do not belong to $\partial \Omega$.

Let $u^{s}(t) \in \partial \Omega$ for $t \in\left[t_{1}, t_{2}\right]$ be a measurable function such that $A u^{s}(t)=(1,0)^{T}$ for a.e. $t \in\left[t_{1}, t_{2}\right]$. Then for the initial point $q_{0}=$ Id there exists a unique corresponding singular extremal of the form $h_{1}=\cos \Omega^{\circ} \theta^{\circ}=$ const, $h_{2}=\sin _{\Omega^{\circ}} \theta^{\circ}=$ const, $h_{3}=0$ and $u(t)=u^{s}(t)$, where $q(t)$ is found as a unique solution to (5.11) with $u(t)=u^{s}(t)$. Moreover, any singular extremal with given $\theta^{\circ}=$ const has the described form. Hence, solutions of linear system (5.25) play a key role in investigating singular extremals.

Let us now investigate linear system (5.25). Obviously, $\operatorname{rk} A \geq 1$, since $\left(\cos \Omega^{\circ} \theta^{\circ}, \sin _{\Omega^{\circ}} \theta^{\circ}\right) \neq 0$. If $\operatorname{rk} A=2$, then linear system (5.25) has a unique solution $u^{s}$. If $u^{s} \notin \partial \Omega$ then there are no singular extremals with given $\theta^{\circ}$. If $u^{s} \in \partial \Omega$, then for the initial point $q_{0}=\mathrm{Id}$, there exists a unique corresponding singular extremal with given $\theta^{\circ}$, and it has the constant singular control $u(t)=u^{s}=$ const.

Let us now consider the special case $\operatorname{rk} A=1$. In this case, system (5.25) has a solution iff the second row of $A$ is $(0,0)$, i.e., $a \sin _{\Omega^{\circ}} \theta^{\circ}=b \cos \Omega^{\circ} \theta^{\circ}=0$.

- If $a^{2}+b^{2}=2$ (cases $\mathfrak{s l}_{2}$ and $\left.\mathfrak{s u}_{2}\right)$, then there are no singular extremals in the special case.

- If $a=0$ and $b=1$ (case $L=\mathfrak{s h}_{2}$ ), then there are singular extremals in the special case if $\cos _{\Omega^{\circ}} \theta^{\circ}=0$ and $\left(0, \sin _{\Omega^{\circ}} \theta^{\circ}\right) \in \mathfrak{S}_{2}$. Indeed, if the last two conditions are fulfilled, then linear system (5.25) has infinite number of solutions, which form a horizontal supporting line to $\Omega$. Intersection of this line with $\partial \Omega$ is a segment $\left[u_{l}^{s}, u_{r}^{s}\right]$ (or a point $u_{l}^{s}=u_{r}^{s}$ if $\left(h_{1}, h_{2}\right) \in \mathfrak{S}_{2} \backslash \mathfrak{S}_{1}$ ). Hence, for any measurable function $u^{s}(t) \in$ $\left[u_{l}^{s}, u_{r}^{s}\right]$ for a.e. $t \in\left[t_{1}, t_{2}\right]\left(\right.$ i.e., $u^{s}(t)=\left(\cos _{\Omega} \theta(t), \sin _{\Omega} \theta(t)\right)$ where $\theta(t) \leftrightarrow \theta^{\circ}$ for a.e. $\left.t \in\left[t_{1}, t_{2}\right]\right)$, there exists a unique corresponding singular extremal with $h_{1}=H \cos _{\Omega^{\circ}} \theta^{\circ}=$ const, $h_{2}=H \sin _{\Omega^{\circ}} \theta^{\circ}=$ const, and $h_{3}=0$.

- If $a=1$ and $b=0$ (case $L=\mathfrak{s e}_{2}$ ), then there are singular extremals in the special case if $\sin _{\Omega^{\circ}} \theta^{\circ}=0$ and $\left(\cos _{\Omega^{\circ}} \theta^{\circ}, 0\right) \in \mathfrak{S}_{2}$. If so, then the situation is similar to the previous one, but for vertical supporting lines to $\Omega$.

- If $a=b=0$ (case $L=\mathfrak{h}_{3}$ ), then there are singular extremals in special case for arbitrary $\theta^{\circ}$ if $\left(\cos _{\Omega^{\circ}} \theta^{\circ}, \sin _{\Omega^{\circ}} \theta^{\circ}\right) \in \mathfrak{S}_{2}$ (see [31]). The corresponding singular control has the form $u^{s}(t)=$ $\left(\cos _{\Omega} \theta(t), \sin _{\Omega} \theta(t)\right)$ where $\theta(t) \leftrightarrow \theta^{\circ}$ for a.e. $t \in\left[t_{1}, t_{2}\right]$.

In the sequel, we distinguish two cases of singular extremals described above:

- general singular extremals in the case $\operatorname{rk} A=2$,

- special singular extremals in the case $\operatorname{rk} A=1$. 


\subsection{Mixed extremals}

A mixed extremal appears when a bang arc $\left(\theta^{\circ}(t), h_{3}(t)\right)$ enters for a finite time a point $\left(\theta_{*}^{\circ}, 0\right)$ that corresponds to a singular extremal.

\subsection{Special case: $\Omega$ a polygon}

Let $\Omega \subset \mathbb{R}^{2}$ be a convex polygon containing the origin in its interior. The polar set to $\Omega$ is a convex polygon $\Omega^{\circ}=\operatorname{conv}\left(\omega_{1}^{\circ}, \ldots, \omega_{k}^{\circ}\right) \subset \mathbb{R}^{2}$ with the origin in its interior. The generalized trigonometric functions $\cos _{\Omega^{\circ}} \theta^{\circ}$ and $\sin _{\Omega^{\circ}} \theta^{\circ}$ are piecewise linear on $\mathbb{R} /\left(2 \mathbb{S}^{\circ} \mathbb{Z}\right)$ with possible corner points $\theta_{1}^{\circ}, \ldots, \theta_{k}^{\circ}$, where $\left(\cos _{\Omega^{\circ}} \theta_{i}^{\circ}, \sin _{\Omega^{\circ}} \theta_{i}^{\circ}\right)=$ $\omega_{i}^{\circ}, i=1, \ldots, k$. The potential energy $\mathcal{U}\left(\theta^{\circ}\right)=\frac{1}{2}\left(a \sin _{\Omega^{\circ}}^{2} \theta^{\circ}-b \cos _{\Omega^{\circ}}^{2} \theta^{\circ}\right)$ is piecewise quadratic, thus $\mathcal{U}^{\prime}\left(\theta^{\circ}\right)$ is piecewise linear with the same possible corner points. So at each segment $\theta^{\circ} \in\left(\theta_{i}^{\circ}, \theta_{i+1}^{\circ}\right), i=1, \ldots, k, \theta_{k+1}^{\circ}=\theta_{1}^{\circ}$, system (5.15) takes the form

$$
\left(\begin{array}{c}
\dot{\theta^{\circ}} \\
\dot{h}_{3}
\end{array}\right)=\left(\begin{array}{cc}
0 & 1 \\
\alpha_{i} & 0
\end{array}\right)\left(\begin{array}{c}
\theta^{\circ} \\
h_{3}
\end{array}\right)+\left(\begin{array}{c}
0 \\
\beta_{i}
\end{array}\right), \quad \alpha_{i}, \beta_{i} \in \mathbb{R}
$$

where $\alpha_{i}=a \cos _{\Omega}^{2} \theta_{i}+b \sin _{\Omega}^{2} \theta_{i}$. This system in the strip $\left(\theta_{i}^{\circ}, \theta_{i+1}^{\circ}\right) \times \mathbb{R}$ has the following phase portraits (we mention in parentheses the elementary functions in which the system is integrated):

$\alpha_{i}>0$ : hyperbolic (hyperbolic functions),

$\alpha_{i}<0$ : elliptic (trigonometric functions),

$\alpha_{i}=0$ : parabolic (quadratic functions).

The phase portrait of system (5.15) in the whole cylinder $\left(\mathbb{R} /\left(2 \mathbb{S}^{\circ} \mathbb{Z}\right)\right) \times \mathbb{R}$ is glued from the phase portraits in the strips $\left(\theta_{i}^{\circ}, \theta_{i+1}^{\circ}\right) \times \mathbb{R}$. Let us examine behaviour of system (5.15) on lines $\theta^{\circ}=\theta_{i}^{\circ}$. If $\theta^{\circ}\left(t_{0}\right)=\theta_{i}^{\circ}$ and $\dot{\theta^{\circ}}\left(t_{0}\right) \neq 0$ on a trajectory, then it intersects the line $\theta^{\circ}=\theta_{i}^{\circ}$ at an isolated point $t=t_{0}$ and has a corner type singularity at it. But if $\theta^{\circ}\left(t_{0}\right)=\theta_{i}^{\circ}$ and $\dot{\theta}^{\circ}\left(t_{0}\right)=0$, then a singular $\operatorname{arc} \theta^{\circ}(t) \equiv \theta_{i}^{\circ}$ with Cauchy nonuniqueness may appear.

Singular arcs are curves $\lambda_{t} \in T^{*} M$ that satisfy conditions (5.20)-(5.24). Switching times correspond to motion of Hamiltonian system (5.15) between successive vertices of the polygon $\Omega^{\circ}$. Note that condition of Theorem 4.6 is automatically fulfilled for each corner $\theta_{0}^{\circ}$ with $0 \in \mathcal{U}^{\prime}\left(\theta_{0}^{\circ}\right)$, since the function $f$ is analytic. So, using Propositions 4.3, 4.5 and Theorem 4.6, we obtain

Theorem 5.5. If $\Omega$ is a convex polygon containing the origin in its interior, then all extremals in problem (5.7)(5.9) are bang-bang, singular or mixed. Hence, if an extremal does not contain special singular control, then the control is piecewise constant and the extremal is piecewise smooth.

In Sections 5.8 and 5.9 we consider in full detail the cases of the squares $\Omega=\left\{u \in \mathbb{R}^{2} \mid\|u\|_{\infty} \leq 1\right\}$ and $\Omega=\left\{u \in \mathbb{R}^{2} \mid\|u\|_{1} \leq 1\right\}$.

\subsection{Special case: $\Omega$ strictly convex}

Let $\Omega \subset \mathbb{R}^{2}$ be a strictly convex compact set containing the origin in its interior. Then the support function $H$ is $C^{1}$ out of the origin, i.e., $\mathfrak{S}_{1}=\{0\}$. If in addition $\partial \Omega^{\circ}$ is $C^{2}$-smooth, then $\vec{H} \in C^{1}$ and there are neither singular nor mixed trajectories. Moreover, if $\partial \Omega^{\circ}$ is $W_{\infty}^{2}$, then the solution is unique by Proposition 4.3. So the following theorem holds.

Theorem 5.6. If $\Omega$ is a strictly convex compact set containing the origin in its interior and $\partial \Omega^{\circ}$ is $C^{k}$ smooth, $k \geq 2$, then all extremals in problem (5.7)-(5.9) are bang and $C^{k}$-smooth. Moreover, there is no Cauchy nonuniqueness of extremals. 


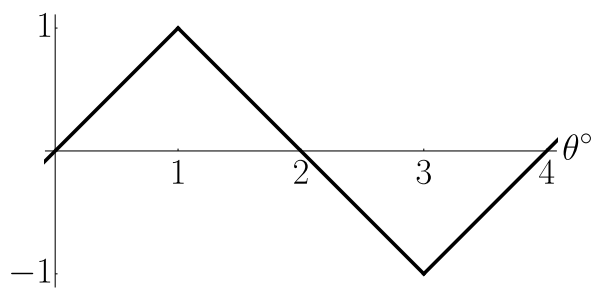

Figure 2. Plot of $\sin _{\Omega^{\circ}} \theta^{\circ}: \ell_{\infty}$.

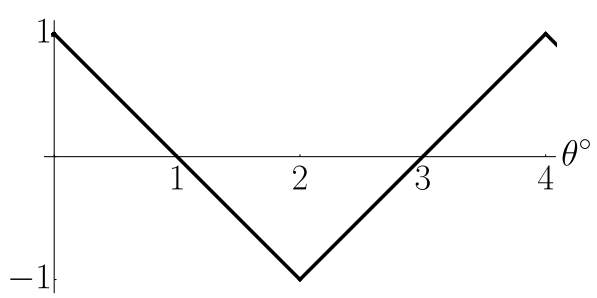

Figure 3. Plot of $\cos _{\Omega^{\circ}} \theta^{\circ}: \ell_{\infty}$.

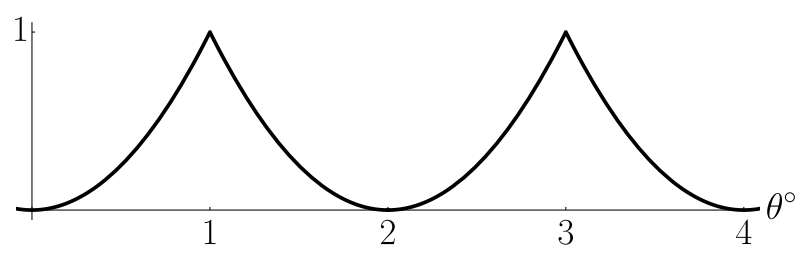

Figure 4. Plot of $\sin _{\Omega^{\circ}}^{2} \theta^{\circ}: \ell_{\infty}$.

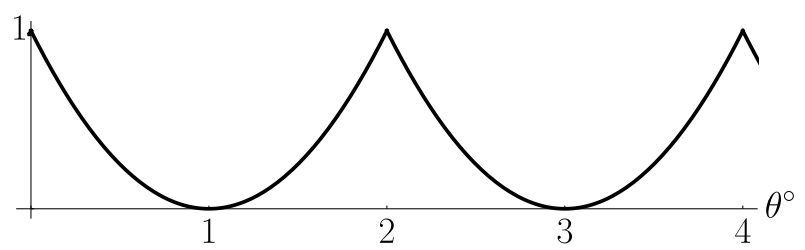

Figure 5. Plot of $\cos _{\Omega^{\circ}}^{2} \theta^{\circ}: \ell_{\infty}$.

Proof. Indeed, $\cos \Omega^{\circ}$ and $\sin _{\Omega^{\circ}}$ are $C^{k}$ functions by Proposition 2.6. Hence, $\mathcal{U}$ is $C^{k}$, and $\theta^{\circ}(t)$ is $C^{k+1}$. Moreover $\theta\left(\theta^{\circ}\right)$ is $C^{k-1}$ by Corollary 2.8. Thus $u_{1}(t)=\cos _{\Omega} \theta\left(\theta^{\circ}(t)\right)$ and $u_{2}(t)=\sin _{\Omega} \theta\left(\theta^{\circ}(t)\right)$ are $C^{k-1}$. Hence extremals are $C^{k}$ by (5.7).

We consider the case $\Omega=\left\{u \in \mathbb{R}^{2} \mid\|u\|_{p} \leq 1\right\}, 1<p<\infty$, in Sect. 5.10.

\subsection{Example: $\Omega=\left\{\|u\|_{\infty} \leq 1\right\}$}

Let $\Omega=\left\{\left(u_{1}, u_{2}\right) \in \mathbb{R}^{2}|| u_{1}|,| u_{2} \mid \leq 1\right\}$. Then $\Omega^{\circ}=\left\{\left(h_{1}, h_{2}\right) \in \mathbb{R}^{2}|| h_{1}|+| h_{2} \mid \leq 1\right\}$ and $\mathbb{S}^{\circ}=2$. The functions $\sin _{\Omega^{\circ}} \theta^{\circ}$ and $\cos \Omega_{\Omega^{\circ}} \theta^{\circ}$ are piecewise linear and 4-periodic, they are plotted at Figures 2 and 3 (these plots were first given in [31]).

We have $\sin _{\Omega^{\circ}}^{2} \theta^{\circ}=\left(1-\left|\left(\theta^{\circ} \bmod 2\right)-1\right|\right)^{2}$, see Figure 4 , and $\cos _{\Omega^{\circ}}^{2} \theta^{\circ}=\left(1-\left(\theta^{\circ} \bmod 2\right)\right)^{2}$, see Figure 5 . Case 1: $a=-1, b=1$. We have $\mathcal{U}\left(\theta^{\circ}+k\right)=-\left(2\left(\theta^{\circ}\right)^{2}-2 \theta^{\circ}+1\right) / 2$ for $\theta^{\circ} \in[0 ; 1], k \in \mathbb{Z}$, see Figure 6 . In the 


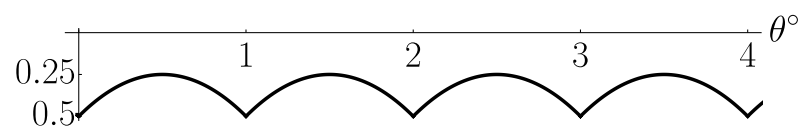

Figure 6 . Plot of $\mathcal{U}\left(\theta^{\circ}\right): \ell_{\infty}$, Case 1.

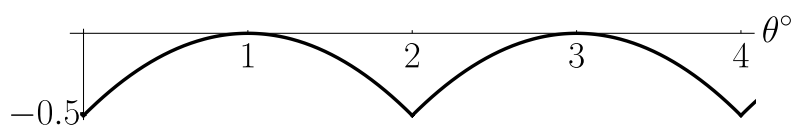

Figure 7. Plot of $\mathcal{U}\left(\theta^{\circ}\right): \ell_{\infty}$, Case 2 .

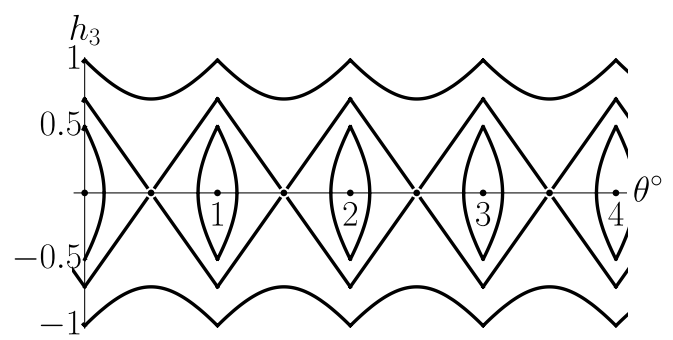

Figure 8. Phase portrait of (5.15): $\ell_{\infty}$, Case 1.

strip $(0,1) \times \mathbb{R}$ system $(5.15)$ has the form $\dot{\theta}^{\circ}=h_{3}, \dot{h}_{3}=2 \theta^{\circ}-1$, it has the saddle phase portrait (see Fig. 8) and the bang trajectories

$$
\begin{aligned}
& \theta^{\circ}=C_{1} \cosh (\sqrt{2} t)+C_{2} \sinh (\sqrt{2} t)+1 / 2, \\
& h_{3}=C_{1} \sqrt{2} \sinh (\sqrt{2} t)+C_{2} \sqrt{2} \cosh (\sqrt{2} t), \\
& C_{1}=\theta_{0}^{\circ}-1 / 2, \quad C_{2}=h_{3}^{0} / \sqrt{2} .
\end{aligned}
$$

General singular extremals are $\left(\theta^{\circ}, h_{3}\right)=(n, 0), n \in \mathbb{Z}$. There are neither mixed nor special singular extremals. Case 2: $a=0, b=1$. We have $\mathcal{U}\left(\theta^{\circ}+2 k\right)=-\left(\theta^{\circ}-1\right)^{2} / 2$ for $\theta^{\circ} \in[0 ; 2], k \in \mathbb{Z}$, see Figure 7 . In the strip $(0,2) \times \mathbb{R}$ system (5.15) has the form $\dot{\theta^{\circ}}=h_{3}, \dot{h}_{3}=\theta^{\circ}-1$, it has the saddle phase portrait (see Fig. 9) and the bang trajectories

$$
\begin{aligned}
& \theta^{\circ}=C_{1} \cosh t+C_{2} \sinh t+1, \\
& h_{3}=C_{1} \sinh t+C_{2} \sinh t, \\
& C_{1}=\theta_{0}^{\circ}-1, \quad C_{2}=h_{3}^{0} .
\end{aligned}
$$

General singular extremals are $\left(\theta^{\circ}, h_{3}\right)=(2 n, 0), n \in \mathbb{Z}$, and special singular extremals are $\left(\theta^{\circ}, h_{3}\right)=(2 n+$ $1,0), n \in \mathbb{Z}$. There are no mixed extremals.

Case 3: $a=1, b=1$. We have $\mathcal{U}\left(\theta^{\circ}+2 k\right)=1 / 2-\left|\theta^{\circ}-1\right|$ for $\theta^{\circ} \in[0 ; 2], k \in \mathbb{Z}$, see Figure 10. In the strip $(0,1) \times \mathbb{R}$ system $(5.15)$ has the form $\dot{\theta^{\circ}}=h_{3}, \dot{h}_{3}=-1$, it has the parabolic phase portrait (see Fig. 12) and the bang trajectories

$$
\begin{aligned}
& \theta^{\circ}=\theta_{0}^{\circ}+h_{3}^{0} t-t^{2} / 2, \\
& h_{3}=h_{3}^{0}-t .
\end{aligned}
$$




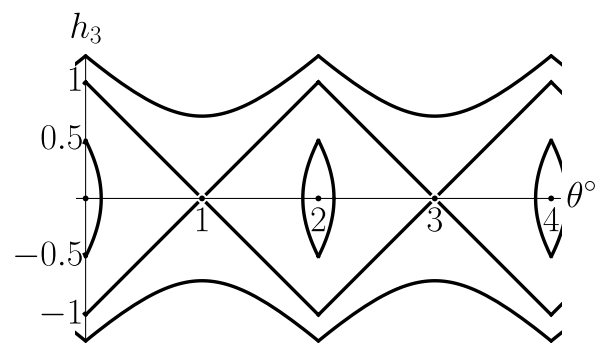

Figure 9. Phase portrait of $(5.15): \ell_{\infty}$, Case 2 .

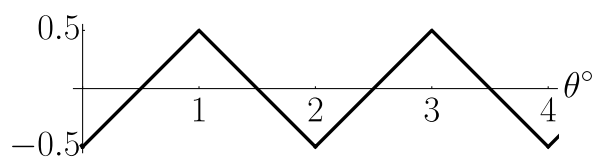

Figure 10. Plot of $\mathcal{U}\left(\theta^{\circ}\right): \ell_{\infty}$, Case 3 .

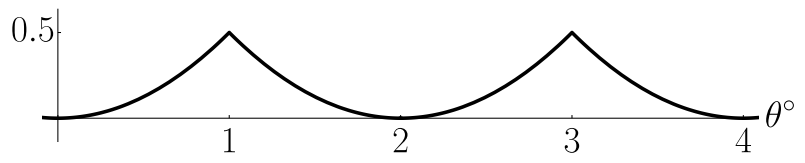

Figure 11. Plot of $\mathcal{U}\left(\theta^{\circ}\right): \ell_{\infty}$, Case 4 .

General singular extremals are $\left(\theta^{\circ}, h_{3}\right)=(n, 0), n \in \mathbb{Z}$. There are no special singular extremals. The parabolas enter the points $\left(\theta^{\circ}, h_{3}\right)=(1,0)$ and $(3,0)$ for finite time. Thus at these points bang extremals join singular extremals, there appear mixed extremals.

Case 4: $a=1, b=0$. We have $\mathcal{U}\left(\theta^{\circ}+2 k\right)=\left(1-\left|\theta^{\circ}-1\right|\right)^{2} / 2$ for $\theta^{\circ} \in[0 ; 2], k \in \mathbb{Z}$, see Figure 11 . In the strip $(0,1) \times \mathbb{R}$ system $(5.15)$ has the form $\dot{\theta^{\circ}}=h_{3}, \dot{h}_{3}=-\theta^{\circ}$, it has the center phase portrait (see Fig. 13) and the bang trajectories - arcs of circles

$$
\begin{aligned}
& \theta^{\circ}=C_{1} \cos t+C_{2} \sin t, \\
& h_{3}=-C_{1} \sin t+C_{2} \cos t, \\
& C_{1}=\theta_{0}^{\circ}, \quad C_{2}=h_{3}^{0} .
\end{aligned}
$$

General singular extremals are $\left(\theta^{\circ}, h_{3}\right)=(2 n+1,0), n \in \mathbb{Z}$, and special singular extremals are $\left(\theta^{\circ}, h_{3}\right)=$ $(2 n, 0), n \in \mathbb{Z}$. The circles enter the points $\left(\theta^{\circ}, h_{3}\right)=(1,0)$ and $(3,0)$ for finite time. Thus at these points bang extremals join (general) singular extremals, there appear mixed extremals.

Case 5: $a=1, b=-1$. We have $\mathcal{U}\left(\theta^{\circ}+k\right)=\left(\theta^{\circ}\right)^{2}-\theta^{\circ}+1 / 2$ for $\theta^{\circ} \in[0 ; 1], k \in \mathbb{Z}$, see Figure 14 . In the strip $(0,1) \times \mathbb{R}$ system $(5.15)$ has the form $\dot{\theta}^{\circ}=h_{3}, \dot{h}_{3}=1-2 \theta^{\circ}$, it has the center phase portrait (see Fig. 15) and the bang trajectories - arcs of ellipses

$$
\begin{aligned}
& \theta^{\circ}=C_{1} \cos (\sqrt{2} t)+C_{2} \sin (\sqrt{2} t)+1 / 2, \\
& h_{3}=-C_{1} \sqrt{2} \sin (\sqrt{2} t)+C_{2} \sqrt{2} \cos (\sqrt{2} t), \\
& C_{1}=\theta_{0}^{\circ}-1 / 2, \quad C_{2}=h_{3}^{0} / \sqrt{2} .
\end{aligned}
$$




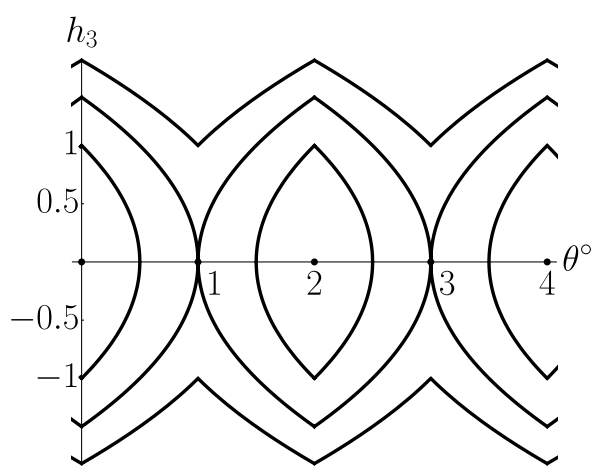

Figure 12. Phase portrait of (5.15): $\ell_{\infty}$, Case 3.

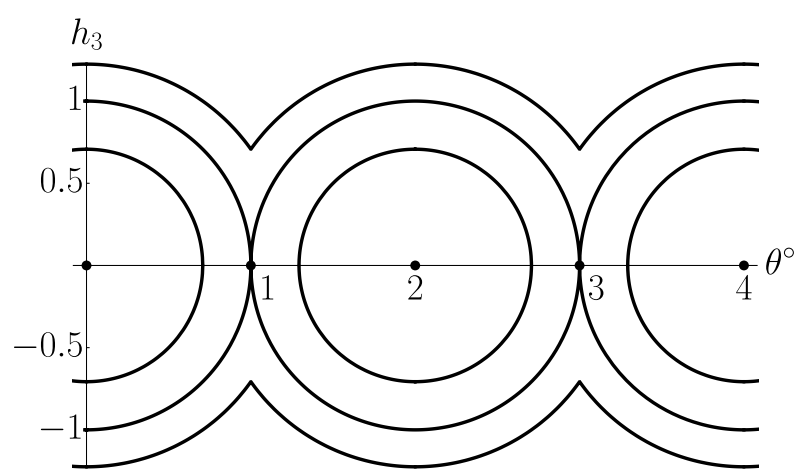

Figure 13. Phase portrait of (5.15): $\ell_{\infty}$, Case 4.

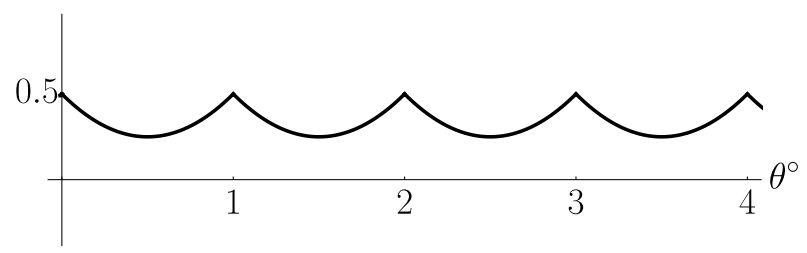

Figure 14. Plot of $\mathcal{U}\left(\theta^{\circ}\right): \ell_{\infty}$, Case 5 .

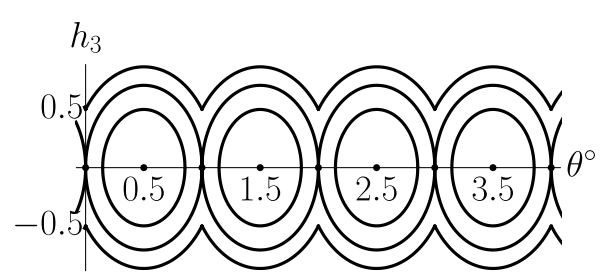

Figure 15. Phase portrait of (5.15): $\ell_{\infty}$, Case 5. 


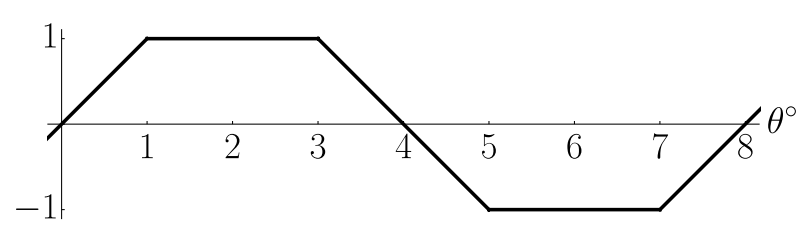

Figure 16. Plot of $\sin _{\Omega^{\circ}} \theta^{\circ}: \ell_{1}$.

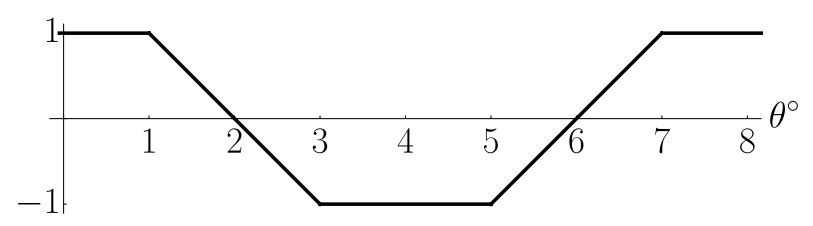

Figure 17. Plot of $\cos \Omega^{\circ} \theta^{\circ}: \ell_{1}$.

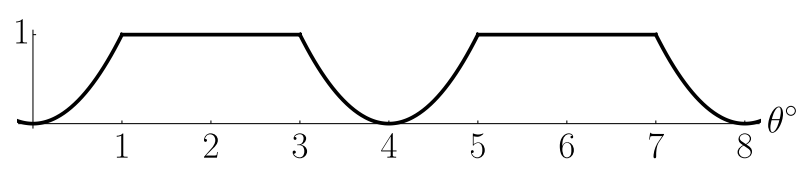

Figure 18. Plot of $\sin _{\Omega^{\circ}}^{2} \theta^{\circ}: \ell_{1}$

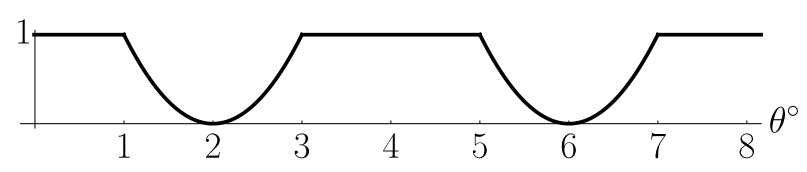

Figure 19. Plot of $\cos _{\Omega^{\circ}}^{2} \theta^{\circ}: \ell_{1}$

General singular extremals are $\left(\theta^{\circ}, h_{3}\right)=(n, 0), n \in \mathbb{Z}$. There are no special singular extremals. The ellipses enter the equilibria $\left(\theta^{\circ}, h_{3}\right)=(n, 0)$ for finite time. Thus at these points bang extremals join singular extremals, there appear mixed extremals.

\subsection{Example: $\Omega=\left\{\|u\|_{1} \leq 1\right\}$}

Let $\Omega=\left\{\left(u_{1}, u_{2}\right) \in \mathbb{R}^{2}|| u_{1}|+| u_{2} \mid \leq 1\right\}$. Then $\Omega^{\circ}=\left\{\left(h_{1}, h_{2}\right) \in \mathbb{R}^{2}|| h_{1}|,| h_{2} \mid \leq 1\right\}$ and $\mathbb{S}\left(\Omega^{\circ}\right)=4$. The functions $\sin _{\Omega^{\circ}} \theta^{\circ}$ and $\cos _{\Omega^{\circ}} \theta^{\circ}$ are piecewise linear and 8-periodic, they are plotted at Figures 16 and 17 (these plots were first given in $[31])$.

The functions $\sin _{\Omega^{\circ}}^{2} \theta^{\circ}, \cos _{\Omega^{\circ}}^{2} \theta^{\circ}$ are 4-periodic, even with respect to $\theta^{\circ}=2$, with

$$
\sin _{\Omega^{\circ}}^{2} \theta^{\circ}=\left\{\begin{array}{ll}
\left(\theta^{\circ}\right)^{2}, & \theta^{\circ} \in[0,1], \\
1, & \theta^{\circ} \in[1,2],
\end{array} \quad \cos _{\Omega^{\circ}}^{2} \theta^{\circ}= \begin{cases}1, & \theta^{\circ} \in[0,1], \\
\left(\theta^{\circ}-2\right)^{2}, & \theta^{\circ} \in[1,2],\end{cases}\right.
$$

see Figures 18 and 19.

Since the set $\Omega$ has neither horizontal nor vertical edges, all singular extremals are of general type (see Sect. 5.4).

Case 1: $a=-1, b=1$. The function $\mathcal{U}\left(\theta^{\circ}\right)$ is 2-periodic and even, with $\mathcal{U}\left(\theta^{\circ}\right)=-\left(1+\left(\theta^{\circ}\right)^{2}\right) / 2$ for $\theta^{\circ} \in[-1,1]$, see Figure 20. In the strip $(0,1) \times \mathbb{R}$ system $(5.15)$ has the form $\dot{\theta^{\circ}}=h_{3}, \dot{h}_{3}=\theta^{\circ}$, it has the saddle phase portrait 


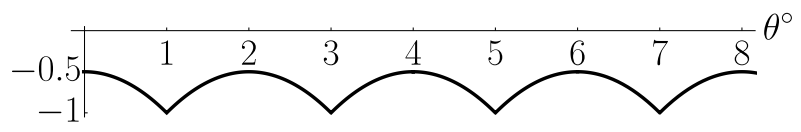

Figure 20. Plot of $\mathcal{U}\left(\theta^{\circ}\right): \ell_{1}$, Case 1.

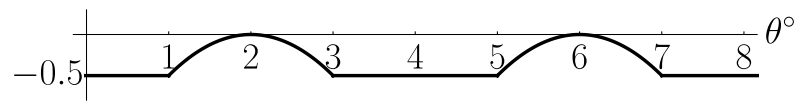

Figure 21. Plot of $\mathcal{U}\left(\theta^{\circ}\right): \ell_{1}$, Case 2 .

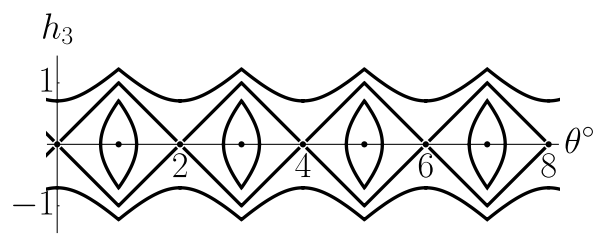

Figure 22. Phase portrait of (5.15): $\ell_{1}$, Case 1.

(see Fig. 22) and the bang trajectories

$$
\begin{aligned}
& \theta^{\circ}=C_{1} \cosh t+C_{2} \sinh t, \\
& h_{3}=C_{1} \sinh t+C_{2} \cosh t, \\
& C_{1}=\theta_{0}^{\circ}, \quad C_{2}=h_{3}^{0} .
\end{aligned}
$$

General singular extremals are $\left(\theta^{\circ}, h_{3}\right)=(2 n+1,0), n \in \mathbb{Z}$. There are no mixed extremals.

Case 2: $a=0, b=1$. The function $\mathcal{U}\left(\theta^{\circ}\right)$ is 4 -periodic and even, with

$$
\mathcal{U}\left(\theta^{\circ}\right)= \begin{cases}-1 / 2, & \theta^{\circ} \in[0,1], \\ -\left(\theta^{\circ}-2\right)^{2} / 2, & \theta^{\circ} \in[1,2],\end{cases}
$$

see Figure 21. In the strip $(0,1) \times \mathbb{R}$ the phase portrait of system (5.15) consists of bang trajectories - horizontal segments $\theta^{\circ}=\theta_{0}^{\circ}+h_{3}^{0} t, h_{3} \equiv h_{3}^{0} \neq 0$ and equilibria $\theta^{\circ} \equiv$ const $\in(0,1), h_{3}=0$. In the strip $(1,2) \times \mathbb{R}$ system (5.15) has the form $\theta^{\circ}=h_{3}, \dot{h}_{3}=\theta^{\circ}-2$, it has the saddle phase portrait (see Fig. 23) and the bang trajectories

$$
\begin{aligned}
& \theta^{\circ}=C_{1} \cosh t+C_{2} \sinh t+2, \\
& h_{3}=C_{1} \sinh t+C_{2} \cosh t, \\
& C_{1}=\theta_{0}^{\circ}-2, \quad C_{2}=h_{3}^{0} .
\end{aligned}
$$

General singular extremals are $\left(\theta^{\circ}, h_{3}\right)=(2 n+1,0), n \in \mathbb{Z}$. There are no mixed extremals.

Case 3: $a=1, b=1$. The function $\mathcal{U}\left(\theta^{\circ}\right)$ is 4-periodic, even and odd w.r.t. $\theta^{\circ}=1$, with $\mathcal{U}\left(\theta^{\circ}\right)=\left(\left(\theta^{\circ}\right)^{2}-1\right) / 2$ for $\theta^{\circ} \in[0,1]$. see Figure 24. In the strip $(0,1) \times \mathbb{R}$ system $(5.15)$ has the form $\dot{\theta}^{\circ}=h_{3}, \dot{h}_{3}=-\theta^{\circ}$, it has the center phase portrait and the bang trajectories - arcs of circles

$$
\theta^{\circ}=C_{1} \cos t+C_{2} \sin t
$$




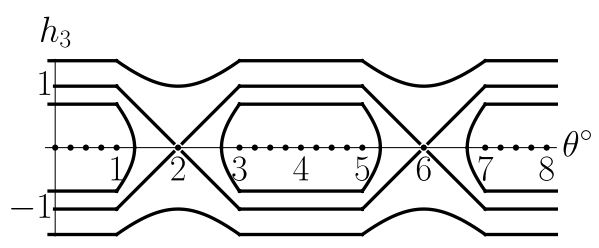

Figure 23. Phase portrait of (5.15): $\ell_{1}$, Case 2 .

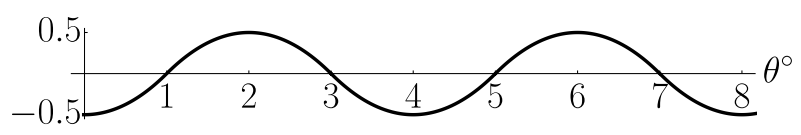

Figure 24. Plot of $\mathcal{U}\left(\theta^{\circ}\right): \ell_{1}$, Case 3 .

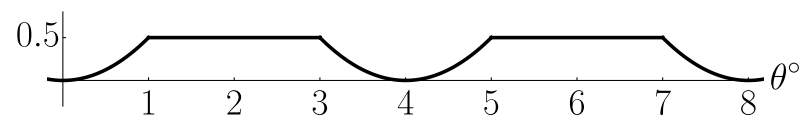

Figure 25. Plot of $\mathcal{U}\left(\theta^{\circ}\right): \ell_{1}$, Case 4 .

$$
\begin{aligned}
& h_{3}=-C_{1} \sin t+C_{2} \cos t, \\
& C_{1}=\theta_{0}^{\circ}, \quad C_{2}=h_{3}^{0} .
\end{aligned}
$$

The circles enter the point $\left(\theta^{\circ}, h_{3}\right)=(1,0)$ for finite time. In the strip $(1,2) \times \mathbb{R}$ system $(5.15)$ has the form $\dot{\theta^{\circ}}=h_{3}, \dot{h}_{3}=\theta^{\circ}-2$, it has the saddle phase portrait (see Fig. 26) and the bang trajectories

$$
\begin{aligned}
& \theta^{\circ}=C_{1} \cosh t+C_{2} \sinh t+2, \\
& h_{3}=C_{1} \sinh t+C_{2} \cosh t, \\
& C_{1}=\theta_{0}^{\circ}-2, \quad C_{2}=h_{3}^{0} .
\end{aligned}
$$

There are neither singular nor mixed extremals.

Case 4: $a=1, b=0$. The function $\mathcal{U}\left(\theta^{\circ}\right)$ is 4-periodic and even, with

$$
\mathcal{U}\left(\theta^{\circ}\right)= \begin{cases}\left(\theta^{\circ}\right)^{2} / 2, & \theta^{\circ} \in[0,1], \\ 1 / 2, & \theta^{\circ} \in[1,2],\end{cases}
$$

see Figure 25. In the strip $(0,1) \times \mathbb{R}$ system (5.15) has the form $\dot{\theta^{\circ}}=h_{3}, \dot{h}_{3}=-\theta^{\circ}$, it has the center phase portrait and the bang trajectories - arcs of circles

$$
\begin{aligned}
& \theta^{\circ}=C_{1} \cos t+C_{2} \sin t, \\
& h_{3}=-C_{1} \sin t+C_{2} \cos t, \\
& C_{1}=\theta_{0}^{\circ}, \quad C_{2}=h_{3}^{0} .
\end{aligned}
$$

In the strip $(1,2) \times \mathbb{R}$ the phase portrait of system (5.15) consists of horizontal segments $\theta^{\circ}=\theta_{0}^{\circ}+h_{3}^{0} t, h_{3} \equiv$ $h_{3}^{0} \neq 0$ and equilibria $\theta^{\circ}=$ const $\in(1,2), h_{3} \equiv 0$, see Figure 27 .

General singular extremals are $\left(\theta^{\circ}, h_{3}\right)=(2 n+1,0), n \in \mathbb{Z}$. The circles enter the points $\left(\theta^{\circ}, h_{3}\right)=(2 n+1,0)$ for finite time. Thus at these points bang extremals join singular extremals, there appear mixed extremals. 


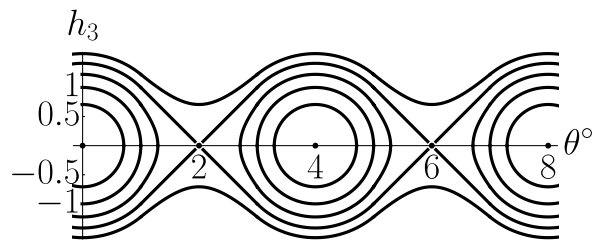

Figure 26. Phase portrait of (5.15): $\ell_{1}$, Case 3.

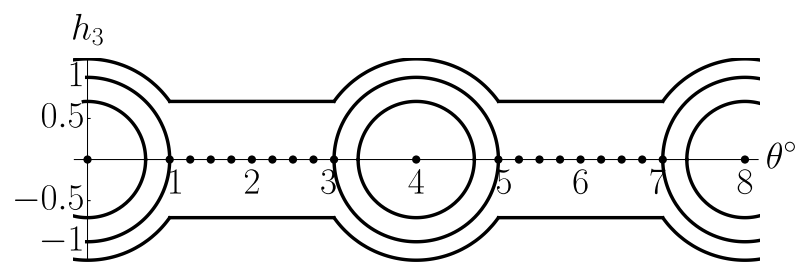

FIGURE 27. Phase portrait of (5.15): $\ell_{1}$, Case 4.

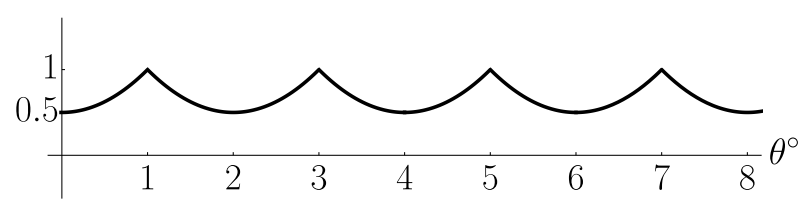

Figure 28. Plot of $\mathcal{U}\left(\theta^{\circ}\right): \ell_{1}$, Case 5 .

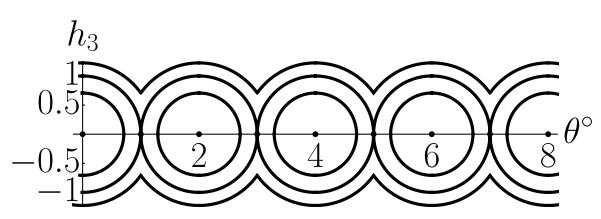

Figure 29. Phase portrait of (5.15): $\ell_{1}$, Case 5 .

Case 5: $a=1, b=-1$. The function $\mathcal{U}\left(\theta^{\circ}\right)$ is 2-periodic and even, with $\mathcal{U}\left(\theta^{\circ}\right)=\left(1+\left(\theta^{\circ}\right)^{2}\right) / 2$ for $\theta^{\circ} \in[0,1]$, see Figure 28. In the strip $(0,1) \times \mathbb{R}$ system (5.15) has the form $\dot{\theta^{\circ}}=h_{3}, \dot{h}_{3}=\theta^{\circ}$, it has the center phase portrait (see Fig. 29) and the bang trajectories - arcs of circles

$$
\begin{aligned}
& \theta^{\circ}=C_{1} \cos t+C_{2} \sin t, \\
& h_{3}=-C_{1} \sin t+C_{2} \cos t, \\
& C_{1}=\theta_{0}^{\circ}, \quad C_{2}=h_{3}^{0} .
\end{aligned}
$$

General singular extremals are $\left(\theta^{\circ}, h_{3}\right)=(2 n+1,0), n \in \mathbb{Z}$. The circles enter the points $\left(\theta^{\circ}, h_{3}\right)=(2 n+1,0)$ for finite time. Thus at these points bang extremals join singular extremals, there appear mixed extremals.

\subsection{Example: $\Omega=\left\{\|u\|_{p} \leq 1\right\}, 1<p<\infty$}

Let $\Omega=\left\{\left.\left(u_{1}, u_{2}\right) \in \mathbb{R}^{2}|| u_{1}\right|^{p}+\left|u_{2}\right|^{p} \leq 1\right\}$, and let $1<p<\infty$, then $\Omega$ is a strictly convex domain. Then $\Omega^{\circ}=\left\{\left.\left(h_{1}, h_{2}\right) \in \mathbb{R}^{2}|| h_{1}\right|^{q}+\left|h_{2}\right|^{q} \leq 1\right\}, q=p /(1-p) \in(1, \infty)$, and $\mathbb{S}^{\circ}=\mathbb{S}\left(\Omega^{\circ}\right)=4 \Gamma^{2}(1+1 / q) / \Gamma(1+2 / q)$. The function $\sin _{\Omega^{\circ}} \theta^{\circ}$ has period $2 \mathbb{S}^{\circ}$, is odd w.r.t. $\theta^{\circ}=\mathbb{S}^{\circ}$ and even w.r.t. $\theta^{\circ}=\mathbb{S}^{\circ} / 2$. The function $\cos _{\Omega^{\circ}} \theta^{\circ}$ 


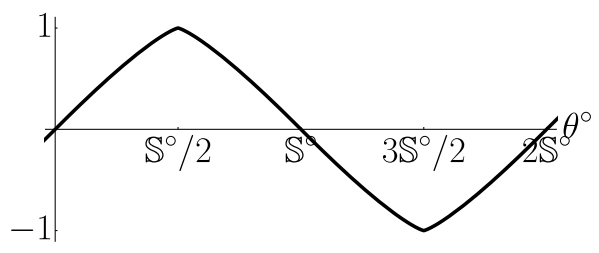

Figure 30. Plot of $\sin _{\Omega^{\circ}} \theta^{\circ}: \ell_{p}, q<2$.

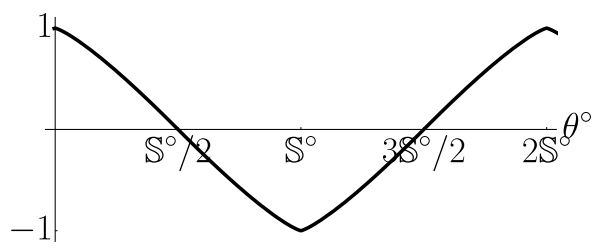

Figure 31. Plot of $\cos _{\Omega^{\circ}} \theta^{\circ}: \ell_{p}, q<2$.

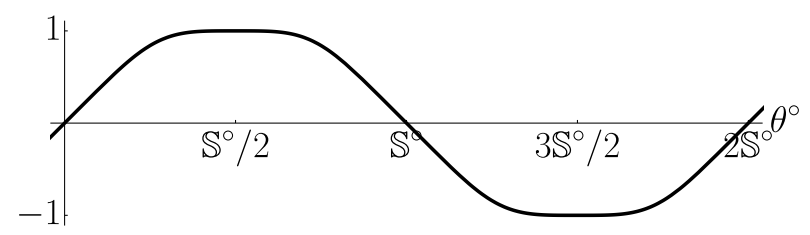

FiguRE 32. Plot of $\sin _{\Omega^{\circ}} \theta^{\circ}: \ell_{p}, q>2$.

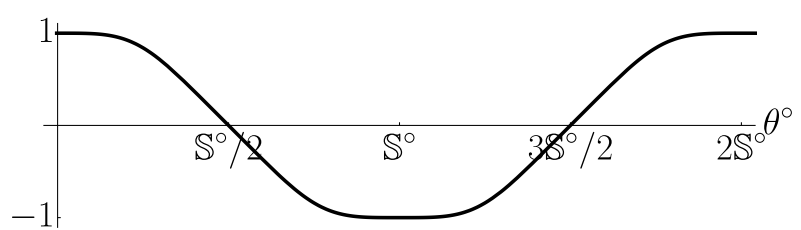

Figure 33. Plot of $\cos _{\Omega^{\circ}} \theta^{\circ}: \ell_{p}, q>2$.

has period $2 \mathbb{S}^{\circ}$, is even w.r.t. $\theta^{\circ}=\mathbb{S}^{\circ}$ and odd w.r.t. $\theta^{\circ}=\mathbb{S}^{\circ} / 2$. If $1<q<2$, then $\sin _{\Omega^{\circ}}\left(\mathbb{S}^{\circ} / 2\right)<1 / \sqrt{2}$ and $\cos _{\Omega^{\circ}}\left(\mathbb{S}^{\circ} / 2\right)<1 / \sqrt{2}$. If $2<q<\infty$, then $\sin _{\Omega^{\circ}}\left(\mathbb{S}^{\circ} / 2\right)>1 / \sqrt{2}$ and $\cos _{\Omega^{\circ}}\left(\mathbb{S}^{\circ} / 2\right)>1 / \sqrt{2}$. The functions $\sin _{\Omega^{\circ}} \theta^{\circ}$ and $\cos _{\Omega^{\circ}} \theta^{\circ}$ are plotted for $q=5 / 4$ at Figures 30 and 31, and for $q=4$ at Figures 32 and 33 .

In the $\ell_{p}$ case the functions $\sin _{\Omega^{\circ}} \theta^{\circ}$ and $\cos _{\Omega^{\circ}} \theta^{\circ}$ are parametrized by hypergeometric functions and system (5.15) seems hard to be explicitly integrable ${ }^{7}$; although, the qualitative structure of the phase portrait is easily constructed below. The sub-Riemannian case $\Omega=\left\{\|u\|_{2} \leq 1\right\}$ leads to the pendulum equation and is integrable in elliptic functions.

The functions $\sin _{\Omega^{\circ}}^{2} \theta^{\circ}, \cos _{\Omega^{\circ}}^{2} \theta^{\circ}$ have period $\mathbb{S}^{\circ}$ and are even. The functions $\sin _{\Omega^{\circ}}^{2} \theta^{\circ}$ and $\cos _{\Omega^{\circ}}^{2} \theta^{\circ}$ are plotted for $q=5 / 4$ at Figures 34 and 35, and for $q=4$ at Figures 36 and 37.

In the case $q \geq 2$ the boundary $\partial \Omega^{\circ}$ and the Hamiltonian $H$ are $C^{2}$-smooth. By Th. 5.6, all extremals are bang and smooth. There is no Cauchy nonuniqueness of extremals.

\footnotetext{
${ }^{7}$ Nonetheless it is Liouville integrable as we have shown.
} 


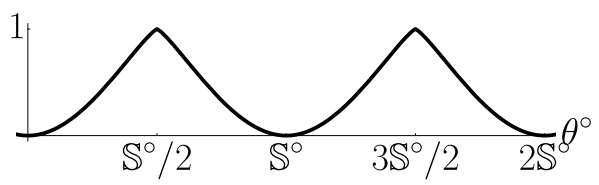

Figure 34. Plot of $\sin _{\Omega^{\circ}}^{2} \theta^{\circ}: \ell_{p}, q<2$.

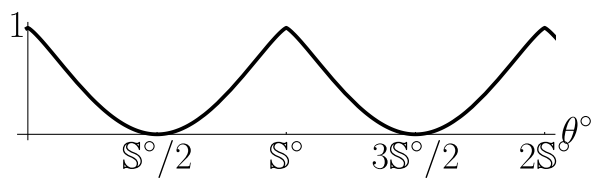

Figure 35. Plot of $\cos _{\Omega^{\circ}}^{2} \theta^{\circ}: \ell_{p}, q<2$.

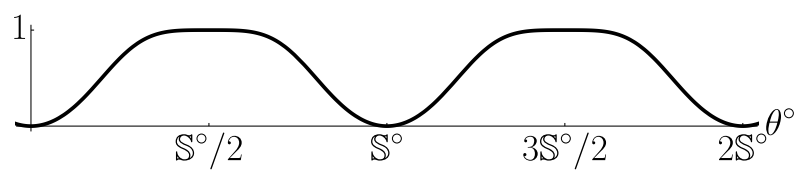

FiguRe 36. Plot of $\sin _{\Omega^{\circ}}^{2} \theta^{\circ}: \ell_{p}, q>2$.

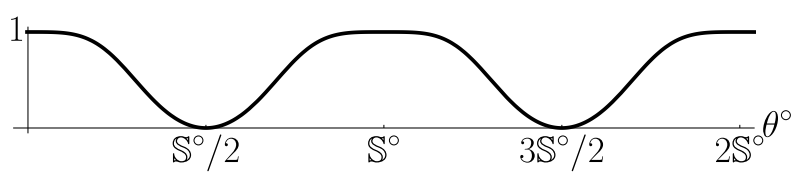

Figure 37. Plot of $\cos _{\Omega^{\circ}}^{2} \theta^{\circ}: \ell_{p}, q>2$.

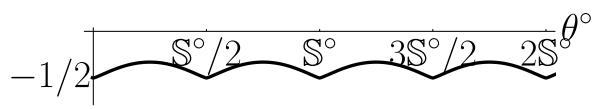

Figure 38. Plot of $\mathcal{U}\left(\theta^{\circ}\right): \ell_{p}, q<2$, Case 1 .

In the case $q \in(1,2)$ the boundary $\partial \Omega^{\circ}$ and the Hamiltonian $H$ are $C^{2}$-smooth if $h_{1} h_{2} \neq 0$, and belong to $C^{1} \backslash C^{2}$ if $h_{1} h_{2}=0, h_{1}^{2}+h_{2}^{2} \neq 0$. In this case there are singular and mixed extremals. Cauchy nonuniqueness due to nonsmoothness appears in some cases, see Th. 5.7 below.

Case 1: $a=-1, b=1$. The function $\mathcal{U}\left(\theta^{\circ}\right)=-\left(\sin _{\Omega^{\circ}}^{2} \theta^{\circ}+\cos _{\Omega^{\circ}}^{2} \theta^{\circ}\right) / 2$ has period $\mathbb{S}^{\circ} / 2$ and is even with respect to $\theta^{\circ}=\mathbb{S}^{\circ} / 4$.

Let $1<q<2$, then $\mathcal{U}\left(\theta^{\circ}\right)$ has minima at $\theta^{\circ}=0$ and $\mathbb{S}^{\circ} / 2$ (which are singular extremals, since $\mathcal{U}\left(\theta^{\circ}\right)$ is not twice differentiable for $\theta^{\circ}=0, \mathbb{S}^{\circ} / 2$ ), and maximum at $\theta^{\circ}=\mathbb{S}^{\circ} / 4$ (this follows from the mutual disposition of the sphere $\Omega^{\circ}$ and the circles $h_{1}^{2}+h_{2}^{2}=$ const), see Figure 38. Thus system (5.15) has center equilibria at $\left(\theta^{\circ}, h_{3}\right)=(0,0)$ and $\left(\theta^{\circ}, h_{3}\right)=\left(\mathbb{S}^{\circ} / 2,0\right)$, and saddle equilibrium at $\left(\theta^{\circ}, h_{3}\right)=\left(\mathbb{S}^{\circ} / 4,0\right)$, see Figure 40 . The function $\mathcal{U}\left(\theta^{\circ}\right)$ is $C^{2}$-smooth when $\theta^{\circ} \neq \mathbb{S}^{\circ} n / 2$, thus the integral $I$ (5.16) diverges, and there is no Cauchy nonuniqueness of extremals. Hence, there are no mixed extremals.

Let $2 \leq q<\infty$, then $\mathcal{U}\left(\theta^{\circ}\right)$ has maxima at $\theta^{\circ}=0$ and $\mathbb{S}^{\circ} / 2$, and minimum at $\theta^{\circ}=\mathbb{S}^{\circ} / 4$, see 39. Thus system $(5.15)$ has saddle equilibria at $\left(\theta^{\circ}, h_{3}\right)=(0,0)$ and $\left(\theta^{\circ}, h_{3}\right)=\left(\mathbb{S}^{\circ} / 2,0\right)$, and center equilibrium at $\left(\theta^{\circ}, h_{3}\right)=\left(\mathbb{S}^{\circ} / 4,0\right)$, see Figure 41.

Case 2: $a=0, b=1$. The function $\mathcal{U}\left(\theta^{\circ}\right)$ has period $\mathbb{S}^{\circ}$ and is even with respect to $\theta^{\circ}=\mathbb{S}^{\circ} / 2$. It has minima 


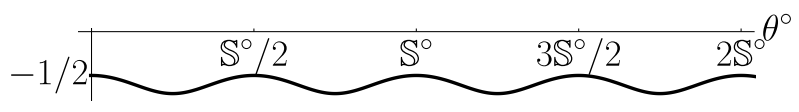

Figure 39. Plot of $\mathcal{U}\left(\theta^{\circ}\right): \ell_{p}, q>2$, Case 1 .

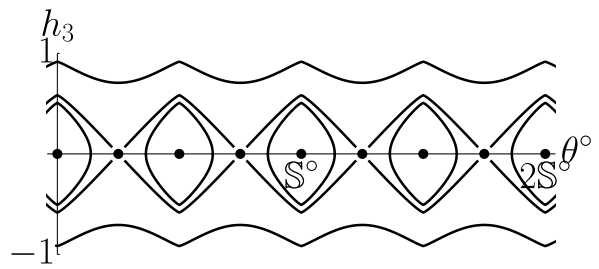

Figure 40. Phase portrait of (5.15): $\ell_{p}, q<2$, Case 1 .

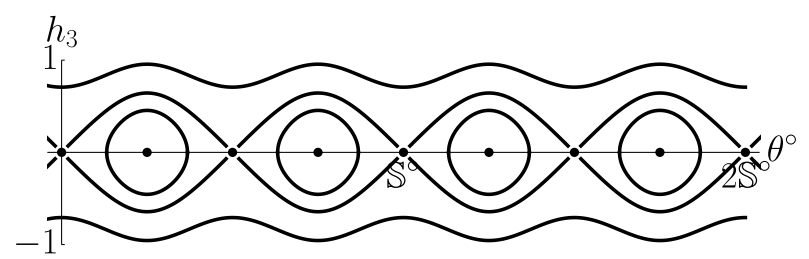

Figure 41. Phase portrait of (5.15): $\ell_{p}, q>2$, Case 1 .

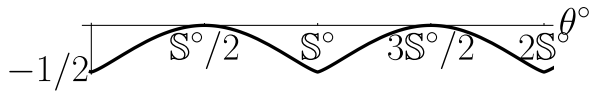

Figure 42. Plot of $\mathcal{U}\left(\theta^{\circ}\right): \ell_{p}, q<2$, Case 2 .

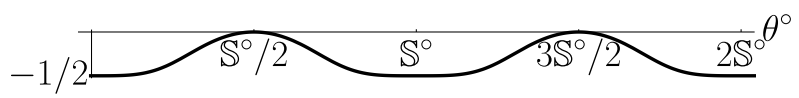

Figure 43. Plot of $\mathcal{U}\left(\theta^{\circ}\right): \ell_{p}, q>2$, Case 2 .

at $\theta^{\circ}=0$ and $\mathbb{S}^{\circ}$, and maximum at $\theta^{\circ}=\mathbb{S}^{\circ} / 2$, see Figures 42,43 . Thus system (5.15) has center equilibria at $\left(\theta^{\circ}, h_{3}\right)=(0,0)$ and $\left(\theta^{\circ}, h_{3}\right)=\left(\mathbb{S}^{\circ}, 0\right)$, and saddle equilibrium at $\left(\theta^{\circ}, h_{3}\right)=\left(\mathbb{S}^{\circ} / 2,0\right)$, see Figures 44,45 .

In the case $1<q<2$ the function $\mathcal{U}\left(\theta^{\circ}\right)$ is $C^{1} \backslash C^{2}$ at $\theta^{\circ}=\mathbb{S}^{\circ} / 2$ (which is a general singular extremal). There is no special singular extremals.. Since

$$
\cos _{\Omega^{\circ}}\left(\theta^{\circ}\right) \sim \mathbb{S}^{\circ} / 2-\theta^{\circ}, \quad \theta^{\circ} \rightarrow \mathbb{S}^{\circ} / 2
$$

then the integral $I$ (5.16) diverges, and there is no Cauchy nonuniqueness of extremals. Hence there are no mixed extremals.

Case 3: $a=1, b=1$. The function $\mathcal{U}\left(\theta^{\circ}\right)=\left(\sin _{\Omega^{\circ}}^{2} \theta^{\circ}-\cos _{\Omega^{\circ}}^{2} \theta^{\circ}\right) / 2$ has period $\mathbb{S}^{\circ}$ and is even with respect to $\theta^{\circ}=\mathbb{S}^{\circ} / 2$. It has minima at $\theta^{\circ}=0$ and $\mathbb{S}^{\circ}$, and maximum at $\theta^{\circ}=\mathbb{S}^{\circ} / 2$, see Figures 46,47 . Thus system $(5.15)$ has center equilibria at $\left(\theta^{\circ}, h_{3}\right)=(0,0)$ and $\left(\theta^{\circ}, h_{3}\right)=\left(\mathbb{S}^{\circ}, 0\right)$, and saddle equilibrium at $\left(\theta^{\circ}, h_{3}\right)=\left(\mathbb{S}^{\circ} / 2,0\right)$ (which are singular extremals), see Figures $48,49$. 


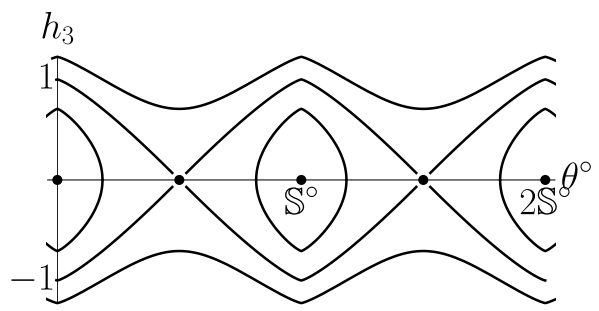

Figure 44. Phase portrait of $(5.15): \ell_{p}, q<2$, Case 2 .

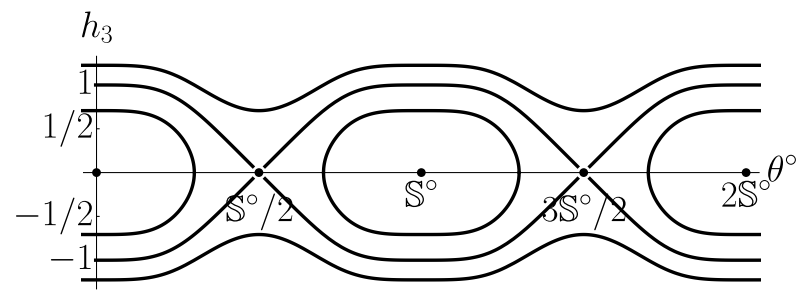

FIgURE 45. Phase portrait of (5.15): $\ell_{p}, q>2$, Case 2 .

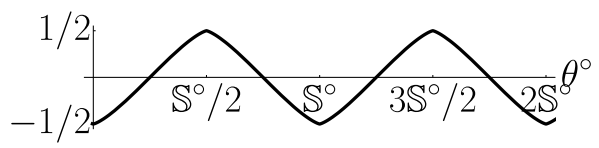

Figure 46. Plot of $\mathcal{U}\left(\theta^{\circ}\right): \ell_{p}, q<2$, Case 3 .

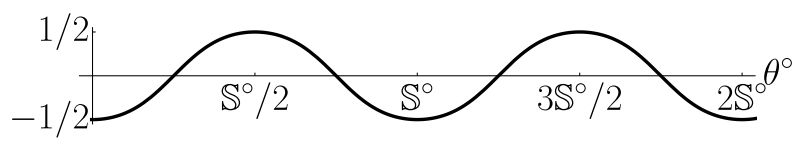

Figure 47. Plot of $\mathcal{U}\left(\theta^{\circ}\right): \ell_{p}, q>2$, Case 3 .

In the case $1<q<2$, the function $\mathcal{U}\left(\theta^{\circ}\right)$ is $C^{1} \backslash C^{2}$ at $\theta^{\circ}=\mathbb{S}^{\circ} / 2$. The asymptotics (5.26) and

$$
\sin _{\Omega^{\circ}}\left(\theta^{\circ}\right)=1-\frac{1}{q}\left(\mathbb{S}^{\circ} / 2-\theta^{\circ}\right)^{q}+O\left(\mathbb{S}^{\circ} / 2-\theta^{\circ}\right)^{2 q}, \quad \theta^{\circ} \rightarrow \mathbb{S}^{\circ} / 2,
$$

imply that the integral $I$ (5.16) converges, and there is Cauchy nonuniqueness of extremals due to nonsmoothness. Hence there are mixed extremals.

Case 4: $a=1, b=0$. The function $\mathcal{U}\left(\theta^{\circ}\right)=\sin _{\Omega^{\circ}}^{2} \theta^{\circ} / 2$ has period $\mathbb{S}^{\circ}$ and is even with respect to $\theta^{\circ}=\mathbb{S}^{\circ} / 2$.

It has minima at $\theta^{\circ}=0$ and $\mathbb{S}^{\circ}$, and maximum at $\theta^{\circ}=\mathbb{S}^{\circ} / 2$, see Figures 50, 51. Thus system (5.15) has center equilibria at $\left(\theta^{\circ}, h_{3}\right)=(0,0)$ and $\left(\theta^{\circ}, h_{3}\right)=\left(\mathbb{S}^{\circ}, 0\right)$, and saddle equilibrium at $\left(\theta^{\circ}, h_{3}\right)=\left(\mathbb{S}^{\circ} / 2,0\right)$, see Figures 52, 53 .

Similarly to Case 3 , if $1<q<2$, there are singular extremals, Cauchy nonuniqueness, and hence mixed extremals.

Case 5: $a=1, b=-1$. The function $\mathcal{U}\left(\theta^{\circ}\right)=\left(\sin _{\Omega^{\circ}}^{2} \theta^{\circ}+\cos _{\Omega^{\circ}}^{2} \theta^{\circ}\right) / 2$ has period $\mathbb{S}^{\circ} / 2$ and is even with respect to $\theta^{\circ}=\mathbb{S}^{\circ} / 4$. 


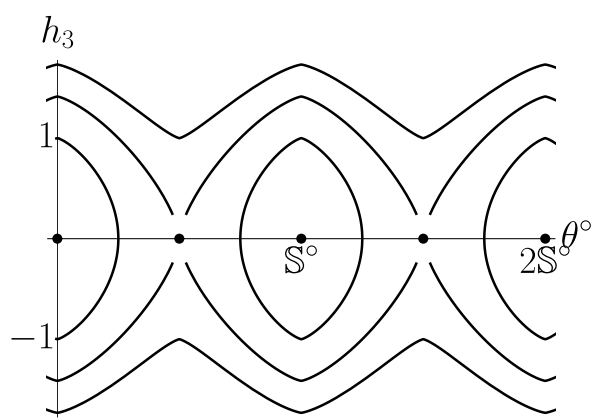

Figure 48. Phase portrait of (5.15): $\ell_{p}, q<2$, Case 3 .

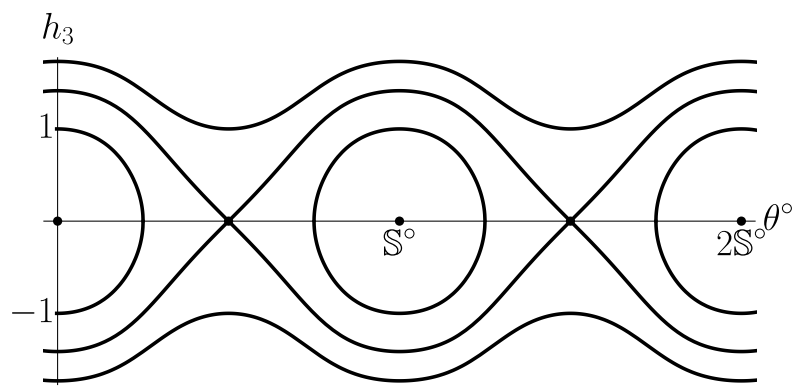

Figure 49. Phase portrait of (5.15): $\ell_{p}, q>2$, Case 3 .

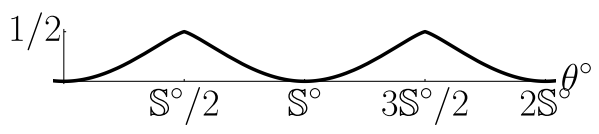

Figure 50. Plot of $\mathcal{U}\left(\theta^{\circ}\right): \ell_{p}, q<2$, Case 4 .

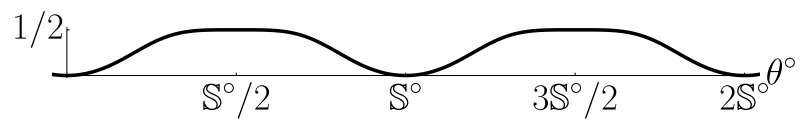

Figure 51. Plot of $\mathcal{U}\left(\theta^{\circ}\right): \ell_{p}, q>2$, Case 4 .

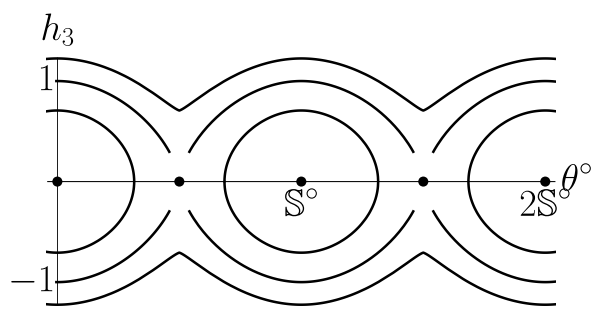

Figure 52. Phase portrait of (5.15): $\ell_{p}, q<2$, Case 4 . 


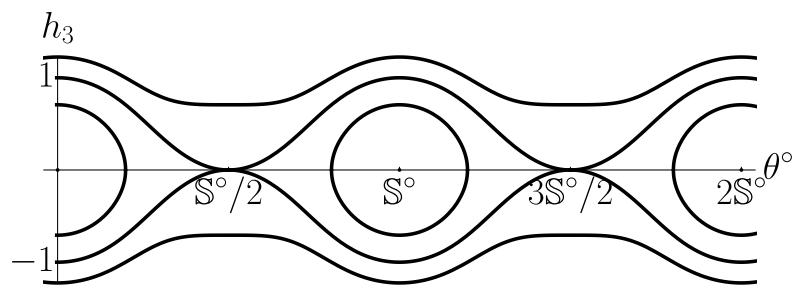

Figure 53. Phase portrait of $(5.15): \ell_{p}, q>2$, Case 4 .

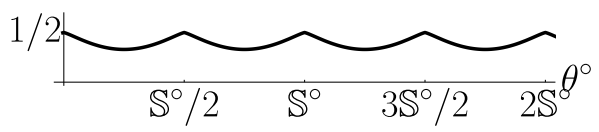

Figure 54. Plot of $\mathcal{U}\left(\theta^{\circ}\right): \ell_{p}, q<2$, Case 5 .

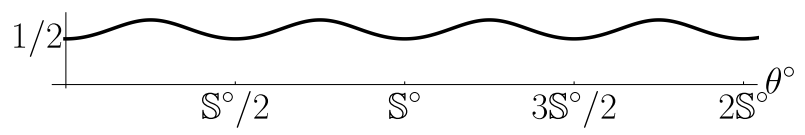

Figure 55. Plot of $\mathcal{U}\left(\theta^{\circ}\right): \ell_{p}, q>2$, Case 5 .

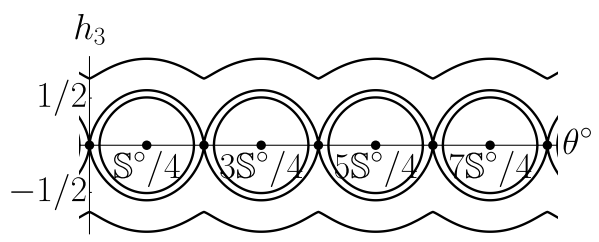

FiguRE 56. Phase portrait of $(5.15): \ell_{p}, q<2$, Case 5 .

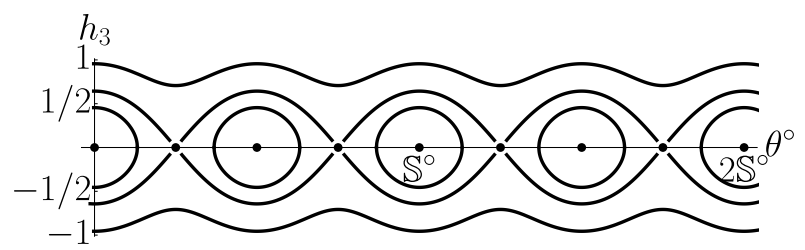

Figure 57. Phase portrait of $(5.15): \ell_{p}, q>2$, Case 5 .

Let $1<q<2$, then $\mathcal{U}\left(\theta^{\circ}\right)$ has maxima at $\theta^{\circ}=0$ and $\mathbb{S}^{\circ} / 2$ (which are singular extremals), and minimum at $\theta^{\circ}=\mathbb{S}^{\circ} / 4$, see 54 . Thus system $(5.15)$ has saddle equilibria at $\left(\theta^{\circ}, h_{3}\right)=(0,0)$ and $\left(\theta^{\circ}, h_{3}\right)=\left(\mathbb{S}^{\circ} / 2,0\right)$, and center equilibrium at $\left(\theta^{\circ}, h_{3}\right)=\left(\mathbb{S}^{\circ} / 4,0\right)$, see Figure 56 .

Similarly to Case 3 , if $1<q<2$, there are Cauchy nonuniqueness, and hence mixed extremals.

Let $2<q<\infty$, then $\mathcal{U}\left(\theta^{\circ}\right)$ has minima at $\theta^{\circ}=0$ and $\mathbb{S}^{\circ} / 2$, and maximum at $\theta^{\circ}=\mathbb{S}^{\circ} / 4$, see 55 . Thus system (5.15) has center equilibria at $\left(\theta^{\circ}, h_{3}\right)=(0,0)$ and $\left(\theta^{\circ}, h_{3}\right)=\left(\mathbb{S}^{\circ} / 2,0\right)$, and saddle equilibrium at $\left(\theta^{\circ}, h_{3}\right)=\left(\mathbb{S}^{\circ} / 4,0\right)$, see Figure 57.

Summing up, we proved the following statement. 
Theorem 5.7. Let $\Omega=\left\{u \in \mathbb{R}^{2} \mid\|u\|_{p}=1\right\}, p \in(1,+\infty)$. If $1<p<2$, then all extremals in problem (5.7)(5.9) are bang. If $p>2$, there are singular extremals. There are Cauchy nonuniqueness and mixed extremals iff $(2<p<\infty$ and $a=1)$.

\section{Rolling of A BALL ON A PLANE}

Another classic problem is about optimal control of a 3D ball, which rolls on a horizontal plane without slipping or twisting. Let us introduce a convenient coordinate system to describe this motion. Denote by $(x, y) \in$ $\mathbb{R}^{2}$ coordinates of the contact point on the plane. We will use quaternions

$$
z=z_{0}+z_{1} \mathbf{i}+z_{2} \mathbf{j}+z_{3} \mathbf{k} \in \mathbb{H} \cong \mathbb{R}^{4}, \quad|z|=1,
$$

to describe rotation of the ball with respect to its initial orientation. The ball is controlled by velocity of its upper point. So we have the following rotation equation:

$$
\dot{z}=\frac{1}{2} z(\dot{y} \mathbf{i}-\dot{x} \mathbf{j})
$$

We assume that the control $\left(u_{1}, u_{2}\right)=(\dot{x}, \dot{y})$ belongs to a compact convex set $\Omega \subset \mathbb{R}^{2}$ and $0 \in$ int $\Omega$ as usual. So we have the following time-minimizing problem:

$$
T \rightarrow \min , \quad \dot{x}=u_{1}, \quad \dot{y}=u_{2}, \quad \dot{z}=\frac{1}{2} z\left(u_{2} \mathbf{i}-u_{1} \mathbf{j}\right), \quad u=\left(u_{1}, u_{2}\right) \in \Omega
$$

with some given end points (for example $x(0)=y(0)=0, z(0)=1$ and $x(T)=x_{f}, y(T)=y_{f}, z(T)=z_{f}$ ). Thus we are searching for a motion such that the ball moves from the initial position $(0,0)$ to the final one $\left(x_{f}, y_{f}\right)$, rotates by $z_{f}$, the velocity of contact point on the plane belongs to $\Omega$, and this motion takes minimal possible time. The contact point during this motion draws on the plane a curve $\gamma$. The motion time is equal to length of $\gamma$ in this Finsler quasimetric on the plane with $\Omega$ as a unit ball. So our time-minimizing problem can be reformulated as follows. Let us call a path connecting $(0,0)$ and $\left(x_{f}, y_{f}\right)$ admissible if the contact point moving along the path rotates the sphere by $z_{f}$. So the problem is to find an admissible path having minimal length in the Finsler quasimetric on the plane. The case when $\Omega$ is the unit Euclidean disc (i.e., we have the Euclidean metric on the plane) was integrated for the first time in [29].

Let us write down the Pontryagin maximum principle. From the Hamiltonian point of view the situation is very simple. We have a left-invariant Hamiltonian system on group $\mathfrak{G}=\mathbb{R}^{2} \times S O(3), \operatorname{dim} \mathfrak{G}=5$. The vertical subsystem is a Hamiltonian system on the Lie coalgebra $\mathfrak{g}^{*}=\mathbb{R}^{2 *} \times s o(3)^{*}$ under the corresponding Lie-Poisson bracket. Since the group $\mathbb{R}^{2}$ is commutative, the rank of the Lie-Poisson bracket on $\mathfrak{g}^{*}$ at a general point is 2 . So we have 3 independent Casimirs on $\mathfrak{g}^{*}$, general symplectic leaves are 2-dimensional, and any Hamiltonian system on $\mathfrak{g}^{*}$ is integrable in Liouville sense. On the whole cotangent bundle $T^{*} \mathfrak{G}$ we have non-degenerate leftand right-invariant symplectic structure. Any left-invariant (smooth) Hamiltonian system is again integrable in Liouville sense, since it always has 5 independent first integrals: the left-invariant Hamiltonian $H$ itself, left (which coincide with right) translations of 3 Casimirs, and right-invariant Hamiltonian $H_{R}$ that is right translation of $\left.H\right|_{\mathfrak{g}^{*}}$ (remind that Hamiltonian coming from PMP is usually nonsmooth).

Despite the written above ideas, we need to write formulas of explicit integration that are simple and convenient to work with. This is also very important, since the Hamiltonian of Pontryagin maximum principle is not smooth, and there can appear singular extremals and Cauchy non-uniqueness phenomenon.

Denote by $(p, q) \in \mathbb{R}^{2}$ the conjugate variables to $(x, y)$ and by $r \in \mathbb{H}$ the conjugate variable to $z$. The dot product of $z$ and $r$ is $\Re(z \bar{r})$, so

$$
\mathcal{H}=p u_{1}+q u_{2}-\frac{1}{2} \Re(z \mathbf{j} \bar{r}) u_{1}+\frac{1}{2} \Re(z \mathbf{i} \bar{r}) u_{2}=\left(p-\frac{1}{2} \Re(z \mathbf{j} \bar{r})\right) u_{1}+\left(q+\frac{1}{2} \Re(z \mathbf{i} \bar{r})\right) u_{2} .
$$


The procedure of integration stays the same as in the previous sections. To find formulas for solutions we write maximum of $\mathcal{H}$ in $u \in \Omega$ in terms of the support function $s_{\Omega}$ of the set $\Omega$ as usual:

$$
H=\max _{u \in \Omega} \mathcal{H}=s_{\Omega}\left(p-\frac{1}{2} \Re(z \mathbf{j} \bar{r}), q+\frac{1}{2} \Re(z \mathbf{i} \bar{r})\right) .
$$

We denote the arguments of $s_{\Omega}$ by $h_{1}=p-\frac{1}{2} \Re(z \mathbf{j} \bar{r})$ and $h_{2}=q+\frac{1}{2} \Re(z \mathbf{i} \bar{r})$. Direct substitution of $\dot{p}=-\mathcal{H}_{x}=0$, $\dot{q}=-\mathcal{H}_{y}=0$ and $\dot{r}=-\mathcal{H}_{z}=\frac{1}{2} r\left(\mathbf{i} u_{2}-\mathbf{j} u_{1}\right)$ gives

$$
\dot{h}_{1}=-h_{3} u_{2}, \quad \dot{h}_{2}=h_{3} u_{1}
$$

where $h_{3}=-\frac{1}{2} \Re(z \mathbf{k} \bar{r})$. Second differentiation gives

$$
\dot{h}_{3}=-\frac{1}{2} \Re(\mathbf{i} z \bar{r}) u_{1}-\frac{1}{2} \Re(\mathbf{j} z \bar{r}) u_{2} .
$$

The right-hand side here is equal to $\left(q-h_{2}\right) u_{1}+\left(h_{1}-p\right) u_{2}$. Consequently, equations on $h_{1}, h_{2}$ and $h_{3}$ do not depend on the other variables and form (together with $\dot{p}=\dot{q}=0$ ) the vertical subsystem:

$$
\dot{h}_{1}=-h_{3} u_{2}, \quad \dot{h}_{2}=h_{3} u_{1}, \quad \dot{h}_{3}=\left(q-h_{2}\right) u_{1}+\left(h_{1}-p\right) u_{2} .
$$

The case $H=0$ leads to abnormal extremals: if $h_{1}=h_{2} \equiv 0$, then $h_{3} \equiv 0,\left(u_{1}, u_{2}\right) \|(p, q)$, and $\left(u_{1}, u_{2}\right) \in \partial \Omega$, which are straight lines in the plane $(x, y)$ independently on $\Omega$.

Let us integrate system (6.2) and find formulas for solutions for the case $H>0$ by using convex trigonometry. Again, since $H=s_{\Omega}\left(h_{1}, h_{2}\right)=$ const, the point $\left(h_{1}, h_{2}\right)$ moves along the boundary of the polar set $\Omega^{\circ}$ stretched by $H$ times:

$$
\left(h_{1}, h_{2}\right) \in H \partial \Omega^{\circ} .
$$

Put

$$
h_{1}=H \cos _{\Omega^{\circ}} \theta^{\circ} \quad \text { and } \quad h_{2}=H \sin _{\Omega^{\circ}} \theta^{\circ} .
$$

If $\left(u_{1}, u_{2}\right)$ is an optimal control, then $h_{1} u_{1}+h_{2} u_{2}=H$ and $u \in \partial \Omega$. So again by the generalized Pythagorean identity (see Sect. 2.2) we get

$$
u_{1}=\cos _{\Omega} \theta \quad \text { and } \quad u_{2}=\sin _{\Omega} \theta
$$

for an angle $\theta \leftrightarrow \theta^{\circ}$. According to the inverse polar change of coordinates (see Sect. 2.2), for a.e. $t$, we have

$$
\dot{\theta}^{\circ}=\frac{h_{1} \dot{h}_{2}-h_{2} \dot{h}_{1}}{H^{2}}=\frac{h_{3}\left(h_{1} u_{1}+h_{2} u_{2}\right)}{H^{2}}=\frac{h_{3}}{H} .
$$

Also from (6.2) we know that

$$
\dot{h}_{3}=\left(q-H \sin _{\Omega^{\circ}} \theta^{\circ}\right) \cos _{\Omega} \theta+\left(H \cos _{\Omega^{\circ}} \theta^{\circ}-p\right) \sin _{\Omega} \theta \text {. }
$$

It is easy to find a first integral for the last two equations using Theorem 4.2. Let us show the direct way to do it (which leads to the same result). If we multiply the second equation by $\dot{\theta^{\circ}}$, then, using the differentiation 
formulae for $\cos \Omega$ and $\sin _{\Omega}$ (see Sect. 2.2), we get

$$
H \ddot{\theta^{\circ}} \dot{\theta^{\circ}}=\dot{h}_{3} \dot{\theta^{\circ}}=\frac{\mathrm{d}}{\mathrm{d} t}\left[p \cos _{\Omega^{\circ}} \theta^{\circ}+q \sin _{\Omega^{\circ}} \theta^{\circ}-\frac{1}{2} H\left(\cos _{\Omega^{\circ}}^{2} \theta^{\circ}+\sin _{\Omega^{\circ}}^{2} \theta^{\circ}\right)\right] .
$$

Thus the first integral has the following form (recall that $p, q$, and $H$ are constants):

$$
\mathbb{E}=\frac{1}{2} H\left({\dot{\theta^{\circ}}}^{2}+\cos _{\Omega^{\circ}}^{2} \theta^{\circ}+\sin _{\Omega^{\circ}}^{2} \theta^{\circ}\right)-p \cos _{\Omega^{\circ}} \theta^{\circ}-q \sin _{\Omega^{\circ}} \theta^{\circ}=\frac{1}{2 H} h_{3}^{2}+H \mathcal{U}\left(\theta^{\circ}\right) .
$$

Let us again emphasize that the control $u=\left(u_{1}, u_{2}\right)$ can easily be found from $\theta^{\circ}$ by the relation $\theta \leftrightarrow \theta^{\circ}$.

If $\Omega$ is the unit disc, then equation (6.5) becomes the classical pendulum equation. In general case, equation (6.5) can be written in terms of kinetic and potential energy $\mathbb{E} / H=\frac{1}{2} \dot{\theta}^{\circ}{ }^{2}+\mathcal{U}\left(\theta^{\circ}\right)$, where $\mathcal{U}$ coincides up to the constant $\frac{p^{2}+q^{2}}{H^{2}}$ with the squared distance from the point $\left(\frac{p}{H}, \frac{q}{H}\right)$ to the point $\left(\cos _{\Omega^{\circ}} \theta^{\circ}, \sin _{\Omega^{\circ}} \theta^{\circ}\right)$ on the boundary $\partial \Omega^{\circ}$. So the topological structure of the phase portrait of equation (6.5) is determined by minima and maxima points on $\partial \Omega^{\circ}$ of the distance to the point $\left(\frac{p}{H}, \frac{q}{H}\right)$. At any local minimum we have a fixed point of the center type, and at any local maximum we have a fixed point of the saddle type.

Theorem 6.1. If problem (6.1) is sub-Riemannian (i.e., the set $\Omega$ is an ellipse centered at the origin), then the vertical subsystem (6.2) for the normal case $H>0$ is integrated in elliptic functions.

Proof. Let $a$ and $b$ be semi-axes of $\Omega$. The polar set $\Omega^{\circ}$ is an ellipse too: $\partial \Omega^{\circ}=\left\{\left(\frac{1}{a} \cos s, \frac{1}{b} \sin s\right)\right\}$. Using formulae obtained in Sect. 2.3, we get $s=a b \theta^{\circ}, \cos _{\Omega^{\circ}} \theta^{\circ}=\frac{1}{a} \cos s$ and $\sin _{\Omega^{\circ}} \theta^{\circ}=\frac{1}{b} \sin s$. Hence, equation (6.5) leads to the following ODE on the parameter $s$ :

$$
\dot{s}=a b \dot{\theta^{\circ}}= \pm \sqrt{\tilde{\mathbb{E}}-b^{2}(\cos s-\tilde{p})^{2}-a^{2}(\sin s-\tilde{q})^{2}} .
$$

Here $\tilde{\mathbb{E}}=\frac{a^{2} b^{2}}{H}\left(2 \mathbb{E} H+p^{2}+q^{2}\right), \tilde{p}=\frac{a p}{H}$, and $\tilde{q}=\frac{b q}{H}$ are some constants. This equation can be integrated explicitly by elliptic functions in a standard way (the explicit form is of no importance here).

Moreover, in the sub-Riemannian case, we immediately find $u_{1}=\cos _{\Omega} \theta=\frac{\mathrm{d}}{\mathrm{d} \theta^{\circ}} \sin _{\Omega^{\circ}} \theta^{\circ}=\frac{\mathrm{d} s}{\mathrm{~d} \theta^{\circ}} \frac{\mathrm{d}}{\mathrm{d} s}\left(\frac{1}{b} \sin s\right)=$ $a \cos s$ and $u_{2}=\sin _{\Omega} \theta=-\frac{\mathrm{d}}{\mathrm{d} \theta^{\circ}} \cos _{\Omega^{\circ}} \theta^{\circ}=-\frac{\mathrm{d} s}{\mathrm{~d} \theta^{\circ}} \frac{\mathrm{d}}{\mathrm{d} s}\left(\frac{1}{a} \cos s\right)=b \sin s$.

If $\Omega^{\circ}$ has $W_{\infty}^{2}$ boundary (in this case $\Omega$ is necessarily strictly convex), then there is a solution uniqueness by Proposition 4.3. Let us now consider the case when $\Omega$ is a polygon.

Theorem 6.2. Suppose that $\Omega$ is a polygon. Then there are two types of normal extremals:

(1) If a control $\hat{u}$ moves arbitrarily along one fixed edge e in $\Omega$, then the obtained extremal is optimal. In this case, the point $\left(\frac{p}{H}, \frac{q}{H}\right)$ coincides with the vertex of $\Omega^{\circ}$ corresponding to the edge $e$.

(2) In the other case, the control $\hat{u}$ is bang-bang (i.e., piecewise constant). The set of its possible values is finite ${ }^{8}$ : a control $\hat{u}=\left(\cos _{\Omega} \hat{\theta}, \sin _{\Omega} \hat{\theta}\right)$ is either a vertex of $\Omega$ or a point on an edge e of $\Omega$, given by the condition

$$
\left(\cos _{\Omega} \hat{\theta}, \sin _{\Omega} \hat{\theta}\right) \|\left(\cos _{\Omega^{\circ}} \theta_{0}^{\circ}-\frac{p}{H}, \sin _{\Omega^{\circ}} \theta_{0}^{\circ}-\frac{q}{H}\right)
$$

where $\theta_{0}^{\circ}$ determines the vertex of $\Omega^{\circ}$ corresponding to edge e. The last case is allowed by PMP if and only if $\mathbb{E}=H \mathcal{U}\left(\theta_{0}^{\circ}\right)$.

\footnotetext{
${ }^{8}$ Only values on a set of positive measure are considered.
} 
Proof. The first part is easy to prove. Indeed, if $\hat{u}(t)$ belongs to a fixed edge $e$ of $\Omega$ for a.e. $t$, then $(x(T), y(T))$ belongs to the boundary of the reachable set (in time $T$ ) for the system $(\dot{x}, \dot{y}) \in \Omega$. So there is no way to reach this point faster, and hence the trajectory is optimal.

The second part follows from Propositions 4.3, 4.5 and Theorem 4.6. Indeed, if an extremal has no singular parts, then the control is piecewise constant. Singular parts are described in Section 4.4. General singular parts are described in item (1a) in Section 4.4, where it is proved that, in this case, solution must stay at a corner, and singular control is uniquely determined by the condition $d f \|\left(\cos _{\Omega} \theta, \sin _{\Omega} \theta\right)$ where $f=\frac{1}{2}\left(x-\frac{p}{H}\right)^{2}+\frac{1}{2}\left(y-\frac{q}{H}\right)^{2}$ for our problem. Special singular controls are described in item (2b) in Section 4.4 and may appear only if $d f=0$ at a corner of $\Omega^{\circ}$ (see Section 4.4). The function $f$ has null derivative $d f(x, y)=0$ only at its global minimum $(x, y)=\left(\frac{p}{H}, \frac{q}{H}\right)$. Thus if $\dot{\theta}^{\circ}(0)=0$, the point $Q=\left(\cos \Omega^{\circ} \theta^{\circ}(0), \sin _{\Omega^{\circ}} \theta^{\circ}(0)\right)$ is a corner of $\Omega^{\circ}$, and $Q=\left(\frac{p}{H}, \frac{q}{H}\right)$, then there exists a unique solution $\theta^{\circ}(t) \equiv$ const, which determines special singular extremals described in the first part.

Applying Remark 4.8 to the current setting, we obtain that a control $\hat{u}=\left(\cos _{\Omega} \hat{\theta}, \sin _{\Omega} \hat{\theta}\right) \in e$ satisfying (6.6) is a general singular control (on the edge $e$ ). If a singular control moves arbitrary along an edge $e$, then it is a special singular control (on the edge $e$ ).

Remark 6.3. On any interval with constant control, the functions $x(t)$ and $y(t)$ are linear. Moreover, if $\Omega$ is a polygon, then it is easy to determine the length of constancy interval. If a control is singular, then the length is arbitrary. In the other case, the point $Q_{\theta^{\circ}}$ moves along an edge of $\Omega^{\circ}$. Since the function $\mathcal{U}$ is quadratic on any edge, the equation $\mathbb{E} / H=\frac{1}{2} \dot{\theta}^{2}{ }^{2}+\mathcal{U}\left(\theta^{\circ}\right)$ has the form

$$
\dot{\theta^{\circ}}= \pm \sqrt{a \theta^{\circ 2}+b \theta^{\circ}+c}
$$

where $a, b$, and $c$ are some constants. Moreover, $a=-\left(\cos _{\Omega}^{2} \theta+\sin _{\Omega}^{2} \theta\right)<0$ for $\theta \leftrightarrow \theta^{\circ}, \theta=$ const. Consequently, this equation can be easily solved in classical trigonometric functions. Hence, the sequence of control jumps can be easily derived from the phase portrait of the equation (6.5), which can be constructed similarly to Section 5 .

\section{YACHTS}

Definition 7.1. Consider an optimal control problem whose differential system is defined by the classic trigonometric functions cos, sin. An $\Omega$-modification of such a problem is the same problem with cos changed to $\cos _{\Omega}$ and $\sin$ to $\sin _{\Omega}$, where a convex compact set $\Omega$ with $0 \in \operatorname{int} \Omega$ defines the modification of the differential system.

Let us state the following control system:

$$
\dot{q}=u_{1} X_{1}+u_{2} X_{2}, \quad q=(x, y, \theta) \in \mathbb{R}_{x, y}^{2} \times\left(\mathbb{R}_{\theta} /(2 \mathbb{S} \mathbb{Z})\right),
$$

with modified vector fields

$$
X_{1}=\left(\cos _{\Omega} \theta, \sin _{\Omega} \theta, 0\right), \quad X_{2}=(0,0,1),
$$

we fix boundary conditions and integral cost functional in general form

$$
\begin{array}{r}
q(0)=q_{0}, \quad q(T)=q_{1}, \\
J=\int_{0}^{T} f\left(u_{1}, u_{2}\right) \mathrm{d} t \rightarrow \min .
\end{array}
$$

Also, we assume a possible restriction on the control

$$
\left(u_{1}, u_{2}\right) \in U \subset \mathbb{R}^{2} .
$$


Remark 7.2. One may consider non-factorized domain for the angle parameter $\theta \in \mathbb{R}_{\theta}$ which does not change extremal controls. However, in the corresponding problem, optimality question can be investigated easier than in the original for some cases. Below, we will refer to such a problem as "non-factorized problem" while concerning optimality of obtained extremal controls.

$\Omega$-modifications of the following four classic optimal control problems appear as specifications of problem (7.1)-(7.5):

- Euler's elastic problem [24] with

$$
U=U_{E}=\left\{\left(u_{1}, u_{2}\right) \in \mathbb{R}^{2} \mid u_{1}=1\right\}, \quad f=f_{E}=\frac{u_{2}^{2}}{2} .
$$

- Markov-Dubins car [23, 33] with

$$
U=U_{M D}=\left\{\left(u_{1}, u_{2}\right) \in \mathbb{R}^{2}\left|u_{1}=1,\right| u_{2} \mid \leq 1\right\} . \quad f=1,
$$

- Reeds-Shepp car [39] with

$$
U=U_{R S}=\left\{\left(u_{1}, u_{2}\right) \in \mathbb{R}^{2}|| u_{1}|\leq 1,| u_{2} \mid \leq 1\right\}, \quad f=1 .
$$

Notice, that originally Reeds and Shepp used condition $\left|u_{1}\right|=1$ instead of $\left|u_{1}\right| \leq 1$, but the corresponding problems are equivalent in the sense of convexity of the set of possible velocities [4].

- sub-Riemannian problem on the group of Euclidean motions of 2-dimensional plane [42] with

$$
U=\mathbb{R}^{2}, \quad f=f_{S R}=\frac{u_{1}^{2}+u_{2}^{2}}{2}
$$

or equivalently with

$$
U=U_{S R}=\left\{\left(u_{1}, u_{2}\right) \in \mathbb{R}^{2} \mid u_{1}^{2}+u_{2}^{2} \leq 1\right\}, \quad f=1 .
$$

All four classic problems express control models for a car-like robot moving on a horizontal plane. $\Omega$ modifications of those problems can be understood as control models for a yacht in a sea (or car moving on a non-horizontal plane). For the first and the second models with $u_{1}=1$ a domain $\Omega$ gives a description of velocity vector at $(x, y)$ as $\left(\cos _{\Omega} \theta, \sin _{\Omega} \theta\right)$. Explicit form of $\Omega$ can be obtained from external disturbances such as wind or water stream (or angle of inclined plane), so $\Omega$ is a disc for no external disturbances. For the third and the forth models we should assume that $\Omega$ is symmetric, i.e., $\Omega=-\Omega$ and yacht can move forward and backward with the same speed. For non-symmetric case with $\Omega \neq-\Omega$ in the third and the forth problems, the physical interpretation fails, however, the mathematical problem follows the same solution presented below.

Remark 7.3. It is possible to set $q_{0}=(0,0,0)$ for all four classic problems. However, in our $\Omega$-modification case we assume $q_{0}=\left(0,0, \theta_{0}\right)$ with $\theta_{0} \in \mathbb{R}_{\theta} /(2 \mathbb{S} \mathbb{Z})$ (even for "non-factorized problem") to emphasize the importance of initial direction $\theta_{0}$ (one may fix it vanishing through rotating of the domain $\Omega$ by the corresponding angle $\theta_{\Omega}^{-1}\left(\theta_{0}\right)$, see Prop. 7.4).

Let us apply PMP to the general problem (7.1)-(7.5) and then proceed with each of its specifications separately. We have the following Hamiltonian function of PMP:

$$
\mathcal{H}=\psi_{0} f\left(u_{1}, u_{2}\right)+\left(\psi_{1} \cos _{\Omega} \theta+\psi_{2} \sin _{\Omega} \theta\right) u_{1}+\psi_{3} u_{2}
$$

where $\psi=\left(\psi_{0}, \psi_{1}, \psi_{2}, \psi_{3}\right)$ is a vector of adjoint variables, here $\psi_{0} \leq 0$ is a constant. 
The vertical subsystem of the Hamiltonian system takes the form:

$$
\left\{\begin{array}{l}
\dot{\psi}_{1}=\dot{\psi}_{2}=0 \\
\dot{\psi}_{3}=\left(\psi_{1} \sin _{\Omega^{\circ}} \theta^{\circ}-\psi_{2} \cos \Omega^{\circ} \theta^{\circ}\right) u_{1} .
\end{array}\right.
$$

The maximality condition depends on the domain $U$ and the function $f\left(u_{1}, u_{2}\right)$ which defines cost functional (7.4). The case $\psi_{1}^{2}+\psi_{2}^{2}=0$ is considered for each problem separately further in the subsections. Suppose $\psi_{1}^{2}+\psi_{2}^{2} \neq 0$. We assume $\psi_{1}^{2}+\psi_{2}^{2}=1$ without loss of generality.

Proposition 7.4 (see [31]). Let $e^{\mathbf{i} \alpha}$ denote the clockwise rotation of $\mathbb{R}^{2}$ by the angle $\alpha \in S^{1}$ around the origin. Then

$$
\begin{aligned}
& \cos _{\Omega} \theta \cos \alpha+\sin _{\Omega} \theta \sin \alpha=\cos _{e^{\mathrm{i} \alpha} \Omega}\left(\theta-\theta_{\Omega}(\alpha)\right), \\
& \sin _{\Omega} \theta \cos \alpha-\cos _{\Omega} \theta \sin \alpha=\sin _{e^{\mathrm{i} \alpha} \Omega}\left(\theta-\theta_{\Omega}(\alpha)\right),
\end{aligned}
$$

where the function $\theta_{\Omega}: S^{1} \rightarrow \mathbb{R} /(2 \mathbb{S Z})$ sets the correspondence between the classic and generalized angles.

Define an angle $\alpha=$ const $\in S^{1}$ satisfying $\psi_{1}=\cos \alpha, \psi_{2}=\sin \alpha$. Using Proposition 7.4 we rewrite vertical subsystem (7.11) as follows:

$$
\dot{\psi}_{3}=\left(\cos \alpha \sin _{\Omega^{\circ}} \theta^{\circ}-\sin \alpha \cos \Omega^{\circ} \theta^{\circ}\right) u_{1}=\sin _{e^{i \alpha} \Omega^{\circ}}\left(\theta^{\circ}-\theta_{\Omega^{\circ}}(\alpha)\right) u_{1} .
$$

With $\tilde{\alpha}^{\circ}=\theta_{\Omega^{\circ}}(\alpha), \tilde{\Omega}^{\circ}=e^{i \alpha} \Omega^{\circ}, \tilde{\theta}^{\circ}=\theta^{\circ}-\tilde{\alpha}^{\circ}$ we have

$$
\dot{\psi}_{3}=u_{1} \sin _{\tilde{\Omega}^{\circ}} \tilde{\theta}^{\circ}
$$

Moreover, with $\tilde{\alpha}=\theta_{\Omega}(\alpha), \tilde{\Omega}=e^{i \alpha} \Omega, \tilde{\theta}=\theta-\tilde{\alpha}$, the Hamiltonian function of PMP takes the following form:

$$
\mathcal{H}=\psi_{0} f\left(u_{1}, u_{2}\right)+u_{1} \cos _{\tilde{\Omega}} \tilde{\theta}+u_{2} \psi_{3} .
$$

For the time minimization problems with $f=1$ we will use the short form of the Hamiltonian function

$$
\mathcal{H}=u_{1} \cos _{\tilde{\Omega}} \tilde{\theta}+u_{2} \psi_{3} .
$$

Now we proceed with each specification for $U$ and $f$ separately.

\subsection{Euler's elastic problem}

We start with an $\Omega$-modification of one of the oldest optimal control problem defined by (7.1)-(7.5) with (7.6). We have $u_{1} \equiv 1$ and we should find $u_{2}$.

Consider the abnormal case $\psi_{0}=0$. From the maximality condition for $\mathcal{H}$ we have $\psi_{3} \equiv 0$, therefore $\psi_{1}^{2}+\psi_{2}^{2} \neq$ 0 and $\dot{\psi}_{3} \equiv 0$ yields $\sin _{\tilde{\Omega}^{\circ}} \tilde{\theta}^{\circ} \equiv 0$ (see (7.13)). Let us interpret this condition geometrically. Since $\tilde{\Omega}$ is compact, there are the minimal value and the maximal value of $\cos _{\tilde{\Omega}} \tilde{\theta}$ denoted by $m_{1}<0$ and $m_{2}>0$. Notice that $\sin _{\tilde{\Omega}^{\circ}} \tilde{\theta}^{\circ} \equiv 0$ iff $\cos _{\tilde{\Omega}} \tilde{\theta} \equiv m_{1}$ or $\cos _{\tilde{\Omega}} \tilde{\theta} \equiv m_{2}$. Therefore, if $\partial \Omega$ contains no edges, then $u_{2} \equiv 0, \forall \alpha \in S^{1}$ with $x(t)=t \cos _{\Omega} \theta_{0}, y(t)=t \sin _{\Omega} \theta_{0}, \theta(t)=\theta_{0}$. Otherwise, for every edge of $\Omega$ with $\theta(t) \in\left[\theta_{-}, \theta_{+}\right]$, we have arbitrary $u_{2}(t)$ for $\theta(t) \in\left(\theta_{-}, \theta_{+}\right)$and $\mp u_{2}(t) \geq 0$ when corresponding $\theta(t)=\theta_{ \pm}$.

Normal case: $\psi_{0}=-1$. From the maximality condition for $\mathcal{H}=-\frac{u_{2}^{2}}{2}+\cos _{\tilde{\Omega}} \tilde{\theta}+u_{2} \psi_{3}$ we have $\dot{\tilde{\theta}}=u_{2}=\psi_{3}$. 

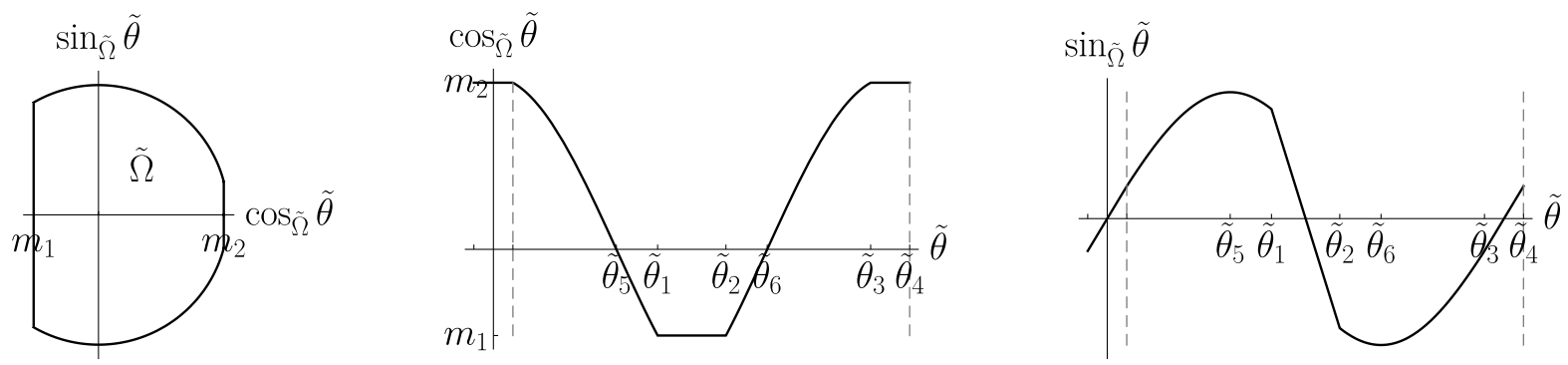

FiguRE 58. An example of $\tilde{\Omega}$ with the corresponding functions $\cos _{\tilde{\Omega}}, \sin _{\tilde{\Omega}}$.

Suppose $\psi_{1}=\psi_{2}=0$. Then $\dot{\psi}_{3} \equiv 0$, so $u_{2}=\psi_{3}=c \equiv$ const, i.e., $\theta=c t+\theta_{0}$. Explicit formulas for $x, y$ can be obtained via integration

$$
x(t)=\frac{1}{c} \int_{\theta_{0}}^{c t+\theta_{0}} \cos \Omega \theta \mathrm{d} \theta, \quad y(t)=\frac{1}{c} \int_{\theta_{0}}^{c t+\theta_{0}} \sin _{\Omega} \theta \mathrm{d} \theta .
$$

If $\psi_{1}^{2}+\psi_{2}^{2} \neq 0$, then we get (7.13) with the maximized Hamiltonian $H=\frac{1}{2} \dot{\tilde{\theta}}^{2}+\cos _{\tilde{\Omega}} \tilde{\theta}$, which determines the phase portrait of the system (see Fig. 59). Since $\tilde{\Omega}$ is compact, there are the minimal value and the maximal value of $\cos _{\tilde{\Omega}} \tilde{\theta}$ denoted by $m_{1}<0$ and $m_{2}>0$ (see Fig. 58 ).

The following cases are possible:

$-H<m_{1}-$ no solutions.

- $H=m_{1} \Rightarrow u_{2}=\dot{\theta} \equiv 0, \cos _{\tilde{\Omega}} \tilde{\theta} \equiv m_{1}$. The corresponding points are $\tilde{\theta} \in \cos _{\tilde{\Omega}}^{-1} m_{1} \equiv\left[\tilde{\theta}_{1}, \tilde{\theta}_{2}\right](\bmod 2 \mathbb{S})$, see Figure 58 at the center. Such points with condition $\dot{\theta}=u_{2}=\psi_{3}=0$ set stable equilibria (see Fig. 59) and on $(x, y)$ we have straight lines with $\theta(t) \equiv \theta_{0} \in\left[\tilde{\theta}_{1}+\tilde{\alpha}, \tilde{\theta}_{2}+\tilde{\alpha}\right]$.

$-H \in\left(m_{1}, m_{2}\right) \Rightarrow \dot{\theta} \not \equiv 0, \cos _{\tilde{\Omega}} \tilde{\theta} \leq H$. There exist two points $\tilde{\theta}_{H}^{-} \not \equiv \tilde{\theta}_{H}^{+}(\bmod 2 \mathbb{S})$, s.t. $\cos _{\tilde{\Omega}} \tilde{\theta}_{H}^{-}=\cos _{\tilde{\Omega}} \tilde{\theta}_{H}^{+}=$ $H$ and $\cos _{\tilde{\Omega}} \tilde{\theta}<H$ for $\tilde{\theta} \in\left(\tilde{\theta}_{H}^{-}, \tilde{\theta}_{H}^{+}\right)$. Here we have periodic behaviour of $\theta$ and quasiperiodic of $x$ and $y$, the corresponding trajectory is called inflectional with inflection points at $\theta(t)=\tilde{\theta}_{H}^{-}+\tilde{\alpha}$ and $\theta(t)=\tilde{\theta}_{H}^{+}+\tilde{\alpha}$ satisfying $\dot{\theta}(t)=0$.

- $H=m_{2}$. Denote $\left[\tilde{\theta}_{3}, \tilde{\theta}_{4}\right] \equiv \cos _{\tilde{\Omega}}^{-1} m_{2}(\bmod 2 \mathbb{S})$. If $\psi_{3} \equiv 0$, then $u_{2}=\dot{\theta} \equiv 0$ and points $\tilde{\theta} \in\left(\tilde{\theta}_{3}, \tilde{\theta}_{4}\right)$ are equilibria, however points $\tilde{\theta} \in\left\{\tilde{\theta}_{3}, \tilde{\theta}_{4}\right\}, \psi_{3}=0$ may be not fixed a priori. If $\psi_{3} \neq 0$, then the corresponding solutions are two separatrices, which can have two types of approaching to points $\tilde{\theta} \in\left\{\tilde{\theta}_{3}, \tilde{\theta}_{4}\right\}$ with $\psi_{3}=0$. If $\tilde{\Omega}$ is $C^{2}$ in the neighborhood of the approaching point with $\tilde{\theta}=\tilde{\theta}_{3}$ or $\tilde{\theta}=\tilde{\theta}_{4}$ then the separatrix is approaching for infinite time to the point, if there is a corner at the corresponding point of $\tilde{\Omega}$ then the separatrix approaches for a finite time (similar to Sect. 5). This case provides the main difference for behaviour of the same system (7.13) in problems 7.1 and 7.2 , see further. This case corresponds to the critical (one loop) elastica for classic formulation of the problem, however, $\Omega$-modification of the problem admits for the corresponding trajectory to have an arbitrary number of separatrices with straight segments (of arbitrary length) in between when $\tilde{\Omega}$ is not $C^{2}$. Detailed analysis of such a situation can be found in Section 4 (up to change $\tilde{\theta}$ to $\theta^{\circ}, H$ to $\mathbb{E}$ and $\cos _{\tilde{\Omega}} \tilde{\theta}$ to $\mathcal{U}\left(\theta^{\circ}\right)$ ).

- $H \in\left(m_{2},+\infty\right) \Rightarrow \psi_{3} \neq 0$, this case provides so-called non-inflectional $(\operatorname{sgn} \dot{\theta} \equiv \pm 1)$ solutions with periodic $\theta$ and quasiperiodic $x$ and $y$.

\subsection{Markov-Dubins problem}

An $\Omega$-modification of Markov-Dubins problem is defined by (7.1)-(7.3) with (7.7), the problem is to find control $u_{2} \in[-1,1]$. 


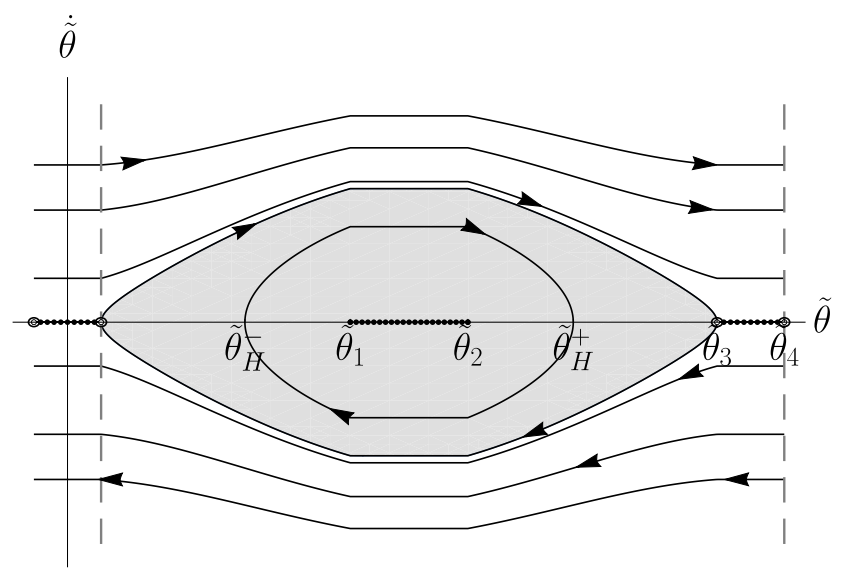

Figure 59. Phase portrait for $\Omega$-modifications of Euler elastica problem in the cylinder $(\tilde{\theta}, \dot{\tilde{\theta}})$ for $\tilde{\Omega}$ presented in Figure 58 .

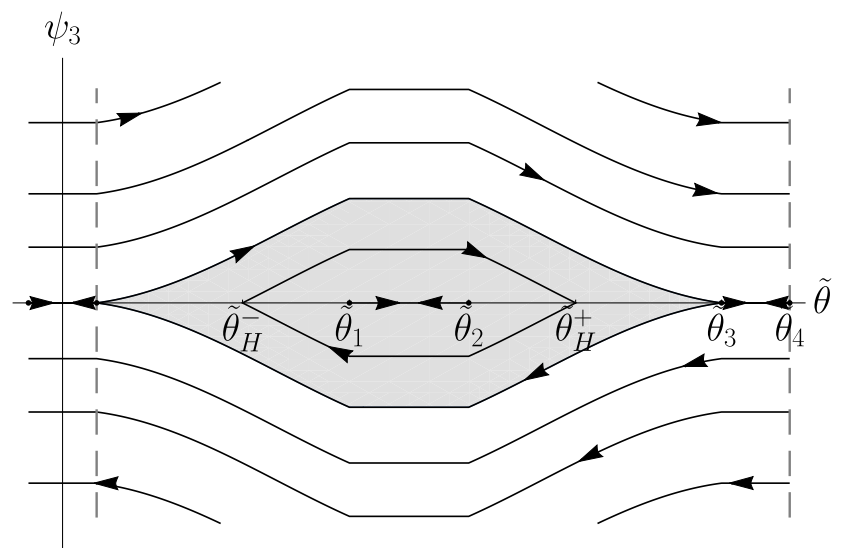

Figure 60 . Phase portrait for $\Omega$-modifications of Markov-Dubins problem in the cylinder $\left(\tilde{\theta}, \psi_{3}\right)$ for $\tilde{\Omega}$ presented in Figure 58 .

Suppose $\psi_{1}=\psi_{2}=0$. Then $\psi_{3} \equiv$ const $\neq 0$ and from the maximum condition for $\mathcal{H}=u_{2} \psi_{3}$ we have $H=\left|\psi_{3}\right|$ and $u_{2}=\operatorname{sgn} \psi_{3} \equiv \pm 1$. The corresponding trajectories on $(x, y)$ depend on the shape of $\Omega$ and can be obtained via integration as (7.16), an example for $\Omega=\tilde{\Omega}$ presented on Figure 58 is given on Figure 61. Those two trajectories always meet at $t=2 \mathbb{S}$ after making one loop for any shape of $\Omega$, an example is given on Figure 61 at the right.

Let $\psi_{1}^{2}+\psi_{2}^{2} \neq 0$. We assume $\psi_{1}^{2}+\psi_{2}^{2}=1$ w.l.o.g. From the maximality condition for $\mathcal{H}(7.15)$ we have

$$
H=\cos _{\Omega} \tilde{\theta}+\left|\psi_{3}\right|, \quad \dot{\tilde{\theta}}=u_{2}=\operatorname{sgn} \psi_{3}= \begin{cases}-1, & \psi_{3}<0, \\ {[-1,1],} & \psi_{3}=0, \\ 1, & \psi_{3}>0\end{cases}
$$

The phase portrait of (7.13) on the cylinder $\left(\tilde{\theta}, \psi_{3}\right)$ is defined by the maximized Hamiltonian $H$ (see Fig. 60 ) and is similar to the one in Euler's elastic problem. Since $\tilde{\Omega}$ is compact, there is a minimal and maximal value of $\cos _{\tilde{\Omega}} \tilde{\theta}$ denoted by $m_{1}<0, m_{2}>0$. The following cases are possible: 

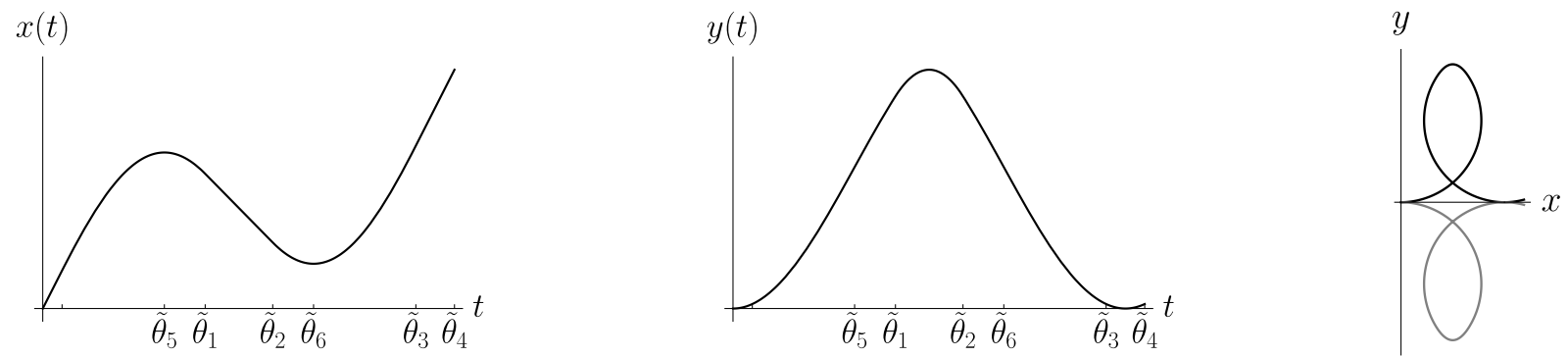

FiguRE 61 . Integrated functions $x(t), y(t)$ for $u_{2} \equiv 1$ and trajectories on $(x, y)$ for $u_{2} \equiv \pm 1$ with $\Omega=\tilde{\Omega}$ given on Figure 58 .

$-H<m_{1}-$ no solutions.

- $H=m_{1} \Rightarrow \psi_{3} \equiv 0, \cos _{\tilde{\Omega}} \tilde{\theta}=m_{1}$. If $\cos _{\tilde{\Omega}}^{-1} m_{1}$ is unique up to periodicity, then there is a unique solution defined by $\tilde{\theta} \equiv \cos _{\tilde{\Omega}}^{-1} m_{1}, u_{2}=0$; otherwise there is a family of solutions $\tilde{\theta}(t) \in \cos _{\tilde{\Omega}}^{-1} m_{1}=\left[\tilde{\theta}_{1}, \tilde{\theta}_{2}\right]$ satisfying $\left|u_{2}(t)\right| \leq 1$ for $\tilde{\theta}(t) \in\left[\tilde{\theta}_{1}, \tilde{\theta}_{2}\right]$ with $u_{2}(t) \geq 0$ at $\tilde{\theta}(t)=\tilde{\theta}_{1}$ and $u_{2}(t) \leq 0$ at $\tilde{\theta}(t)=\tilde{\theta}_{2}$ (see Fig. 60).

- $H \in\left(m_{1}, m_{2}\right), \cos _{\tilde{\Omega}} \tilde{\theta} \leq H \Rightarrow \psi_{3} \not \equiv 0$, since for $\cos _{\tilde{\Omega}} \tilde{\theta}=H$ there holds $\sin _{\tilde{\Omega}^{\circ}} \tilde{\theta}^{\circ} \neq 0$. Moreover $\cos _{\tilde{\Omega}}^{-1} H \equiv$ $\left\{\tilde{\theta}_{H}^{-}, \tilde{\theta}_{H}^{+}\right\}\left(\bmod 2 \mathbb{S}\right.$ ), where $\tilde{\theta}_{H}^{-}<\tilde{\theta}_{1} \leq \tilde{\theta}_{2}<\tilde{\theta}_{H}^{+}$(see Fig. 60). Therefore control $u_{2}$ is switching between 1 and -1 at points $\tilde{\theta} \in\left\{\tilde{\theta}_{H}^{-}, \tilde{\theta}_{H}^{+}\right\}$, the corresponding time intervals are $T_{b}, T_{1}, \ldots, T_{1}, T_{e}$, where $T_{1}=$ $\tilde{\theta}_{H}^{+}-\tilde{\theta}_{H}^{-}<2 \mathbb{S}$ with $T_{b} \leq T_{1}, T_{e} \leq T_{1}$.

- $H=m_{2}$. When $\psi_{3}=0$ we have $\cos _{\tilde{\Omega}} \tilde{\theta}=m_{2}$ for $\tilde{\theta} \in\left[\tilde{\theta}_{3}, \tilde{\theta}_{4}\right](\bmod 2 \mathbb{S})$ (see Fig. 58). In this subcase when $\tilde{\theta} \in\left[\tilde{\theta}_{3}, \tilde{\theta}_{4}\right](\bmod 2 \mathbb{S})$ we have arbitrary $\left|u_{2}(t)\right| \leq 1$ (below, we refer to such a control as uncertain control), it is possible to switch to certain control $u_{2}=-1$ at $\tilde{\theta}=\tilde{\theta}_{3}$ and to certain control $u_{2}=1$ at $\tilde{\theta}=\tilde{\theta}_{4}$. After passing through the switching points $\tilde{\theta}_{3}, \tilde{\theta}_{4}$ we have $\psi_{3} \neq 0$ and control $u_{2}=\operatorname{sgn} \psi_{3} \equiv \pm 1$ for time $T_{1} \equiv\left(\tilde{\theta}_{3}-\tilde{\theta}_{4}\right)(\bmod 2 \mathbb{S})$.

$-H \in\left(m_{2},+\infty\right), \psi_{3} \neq 0$, control $u_{2} \in\{-1,1\}$ is constant for arbitrary time similarly to the case $\psi_{1}=\psi_{2}=$ 0 .

Theorem 7.5. When $\Omega$ is strictly convex, then for any rotated $\tilde{\Omega}$ we have $\tilde{\theta}_{1} \equiv \tilde{\theta}_{2}(\bmod 2 \mathbb{S})$ and $\tilde{\theta}_{3} \equiv \tilde{\theta}_{4}($ mod $2 \mathbb{S})$. Optimal control $u_{2}$ is a piecewise constant function with values $u_{2}^{1}, u_{2}^{2}, \ldots, u_{2}^{n}$; corresponding time intervals $T^{1}, T^{2}, \ldots, T^{n}$ and is one of two types:

$-u_{2}^{2 k}=0, u_{2}^{2 k+1} \in\{-1,1\}, k \in \mathbb{N} ; T^{2 k}, k \in \mathbb{N}$ are arbitrary and $T^{1} \leq 2 \mathbb{S}, T^{n} \leq 2 \mathbb{S}, T^{2 k+1}=2 \mathbb{S}$ for $k \neq$ $0,2 k+1 \neq n$.

$-u_{2}^{2 k}= \pm 1, u_{2}^{2 k+1}=\mp 1, k \in \mathbb{N} ; T^{2}=T^{3}=\cdots=T^{n-1}=\hat{T} \leq 2 \mathbb{S}, T^{1} \leq \hat{T}, T^{n} \leq \hat{T}$.

Theorem 7.6. When $\Omega$ is not strictly convex, then $\tilde{\Omega}$ for some angle $\alpha$ has a vertical side at the right or at the left, as a consequence $\tilde{\theta}_{3} \not \equiv \tilde{\theta}_{4}(\bmod 2 \mathbb{S})$ or $\tilde{\theta}_{1} \not \equiv \tilde{\theta}_{2}(\bmod 2 \mathbb{S})$. This case admits not only optimal piecewise constant control described in Theorem 7.5, but also admits optimal control with uncertain pieces corresponding to the edge $\theta(t) \in\left[\tilde{\theta}_{3}+\tilde{\alpha}, \tilde{\theta}_{4}+\tilde{\alpha}\right]$ or to the edge $\theta(t) \in\left[\tilde{\theta}_{1}+\tilde{\alpha}, \tilde{\theta}_{2}+\tilde{\alpha}\right]$, which are described in case 7.2 or in case 7.2 correspondingly.

In such situation case 7.2 defines strictly singular trajectories. Meanwhile, case 7.2 provides mixed (combination of singular and nonsingular) trajectories.

\subsection{Reeds-Shepp problem}

An $\Omega$-modification of Reeds-Shepp problem is defined by (7.1)-(7.3) with (7.8).

Suppose $\psi_{1}=\psi_{2}=0$. Then $\psi_{3} \equiv$ const $\neq 0$ and from the maximum condition for $\mathcal{H}=u_{2} \psi_{3}$ we have $H=\left|\psi_{3}\right|$ with $u_{2} \equiv \operatorname{sgn} \psi_{3}$. For any choice of $u_{1} \equiv \pm 1$, we get an optimal trajectory since it is not possible to turn on 
such an angle faster (up to infinity for "non-factorized problem"), explicit formulas for $(x, y)$ can be obtained via integration as in (7.16) with $c=1$ for $u_{1} \equiv 1$ and inverse for $u_{1} \equiv-1$.

Let $\psi_{1}^{2}+\psi_{2}^{2} \neq 0$. We assume $\psi_{1}^{2}+\psi_{2}^{2}=1$ w.l.g. From the maximality condition for $\mathcal{H}$ we have

$$
H=\left|\cos _{\tilde{\Omega}} \tilde{\theta}\right|+\left|\psi_{3}\right|, \quad u_{1}=\operatorname{sgn} \cos _{\tilde{\Omega}} \tilde{\theta}, \quad \dot{\tilde{\theta}}=u_{2}=\operatorname{sgn} \psi_{3}= \begin{cases}-1, & \psi_{3}<0 \\ {[-1,1],} & \psi_{3}=0 \\ 1, & \psi_{3}>0\end{cases}
$$

The phase portrait of (7.13) on the cylinder $\left(\tilde{\theta}, \psi_{3}\right)$ is defined by the maximized Hamiltonian $H$. There are only two points $\tilde{\theta}_{5}=\theta_{\tilde{\Omega}}(\pi / 2)$ and $\tilde{\theta}_{6}=\theta_{\tilde{\Omega}}(3 \pi / 2)$ satisfying the condition $\cos _{\tilde{\Omega}} \tilde{\theta}=0$ on $\tilde{\Omega}$ (see Fig. 58). Since $\tilde{\Omega}$ is compact, there is a minimal and maximal value of $\cos _{\tilde{\Omega}} \tilde{\theta}$ denoted by $m_{1}<0, m_{2}>0$. We assume $\left|m_{1}\right| \leq m_{2}$ without loss of generality. Suppose $\cos _{\tilde{\Omega}}^{-1} m_{1}=\left[\tilde{\theta}_{1}, \tilde{\theta}_{2}\right]$ and $\cos _{\tilde{\Omega}}^{-1} m_{2}=\left[\tilde{\theta}_{3}, \tilde{\theta}_{4}\right]$.

The following cases are possible for $H \geq 0$ :

- $H=0$, then $\dot{\theta}=\psi_{3} \equiv 0$ and $\tilde{\theta} \in\left\{\tilde{\theta}_{5}, \tilde{\theta}_{6}\right\}$. We have two fixed points with $u_{1}= \pm 1, u_{2}=0$, the corresponding trajectory on $(x, y)$ is a straight line.

$-H \in\left(0,\left|m_{1}\right|\right)$. From convexity of $\tilde{\Omega}$ we have $\cos _{\tilde{\Omega}}^{-1} H=\left\{\tilde{\theta}_{+H}^{+}, \tilde{\theta}_{+H}^{-}\right\}$and $\cos _{\tilde{\Omega}}^{-1}(-H)=\left\{\tilde{\theta}_{-H}^{+}, \tilde{\theta}_{-H}^{-}\right\}$. This subcase gives us two families of trajectories with $\tilde{\theta} \in\left[\tilde{\theta}_{+H}^{+}, \tilde{\theta}_{-H}^{+}\right]$and $\tilde{\theta} \in\left[\tilde{\theta}_{-H}^{-}, \tilde{\theta}_{+H}^{-}\right]$. Consider the first one without loss of generality. The control $\left(u_{1}, u_{2}\right)$ is switching as follows: $\ldots,(1,1),(-1,1),(-1,-1),(1,-1), \ldots$ The corresponding time intervals $T_{(1,1)}, T_{(-1,1)}, T_{(-1,-1)}, T_{(1,-1)}$ satisfy $T_{(1,1)}=T_{(1,-1)}=\tilde{\theta}_{5}-\tilde{\theta}_{+H}^{+}, T_{(-1,1)}=T_{(-1,-1)}=\tilde{\theta}_{-H}^{+}-\tilde{\theta}_{5}$ (see Fig. 62). The first and the last control can be one of the four types with shortened time intervals $T_{b}, T_{e}$.

- $H=\left|m_{1}\right|$. If $\tilde{\theta} \in\left[\tilde{\theta}_{1}, \tilde{\theta}_{2}\right](\bmod 2 \mathbb{S})$ we have $u_{1}(t)=-1$ and arbitrary $\left|u_{2}(t)\right| \leq 1$ (an uncertain control), it is possible to switch to certain control $u_{2}=-1$ while passing through $\tilde{\theta}=\tilde{\theta}_{1}$ and to certain control $u_{2}=1$ while passing through $\tilde{\theta}=\tilde{\theta}_{2}$, the time intervals corresponding to these certain controls are equal to $\tilde{\theta}_{1}-\tilde{\theta}_{5}$ and $\tilde{\theta}_{6}-\tilde{\theta}_{2}$, then we have 3 more switchings to a certain controls according to Figure 62 similarly to case 7.3. After making full loop we go back to the points $\tilde{\theta}_{1}, \tilde{\theta}_{2}$, where we can switch to uncertain control again staying in $\left[\tilde{\theta}_{1}, \tilde{\theta}_{2}\right]$ or go for another full loop with certain controls.

- $H \in\left(\left|m_{1}\right|, m_{2}\right)$, this case can be treated in the same way as case 7.3. The control $\left(u_{1}, u_{2}\right)$ is switching in a sequence as follows: ..., $(1,1),(-1,1),(1,1),(1,-1),(-1,-1),(1,-1), \ldots$ (further repeating with the same pattern). The corresponding time intervals in the pattern are .., $T_{1}, T_{2}, T_{3}, T_{3}, T_{2}, T_{1}, \ldots$, where $T_{3}=\tilde{\theta}_{6}-\tilde{\theta}_{5}$ and values of $T_{1}, T_{2}$ depend on points $\cos _{\tilde{\Omega}}^{-1} H$.

- $H=m_{2}$, this case can be treated in the same way as case 7.3. Here we have an uncertain control $u_{2}(t) \in$ $[-1,1]$ for interval $\tilde{\theta}(t) \in\left[\tilde{\theta}_{3}, \tilde{\theta}_{4}\right]$ with $u_{1}(t)=1$. Passing through the point $\tilde{\theta}_{3}$ the control $u$ is switching according to certain pattern $(1,-1),(-1,-1),(1,-1)$ with the corresponding time intervals $T_{1}=\tilde{\theta}_{3}-$ $\tilde{\theta}_{6}, T_{3}=\tilde{\theta}_{6}-\tilde{\theta}_{5}, T_{2}=\tilde{\theta}_{5}-\tilde{\theta}_{4}(\bmod 2 \mathbb{S})$. Passing through the point $\tilde{\theta}_{4}$ the control $u$ is switching to certain pattern $(1,1),(-1,1),(1,1)$ with the time intervals $T_{2}, T_{3}, T_{1}$.

- $H \in\left(m_{2},+\infty\right)$ with $\psi_{3} \neq 0$ we have $u_{2} \equiv \pm 1$, the other control $u_{1}$ is switching between 1 and -1 with the corresponding time intervals $T_{+}=\tilde{\theta}_{6}-\tilde{\theta}_{5}, T_{-}=2 \mathbb{S}-T_{+}$.

Remark 7.7. When $\left|m_{1}\right|=m_{2}$ we are missing case 7.3 and cases $7.3,7.3$ join in the one. It provides two uncertain intervals $\tilde{\theta} \in\left[\tilde{\theta}_{1}, \tilde{\theta}_{2}\right] \cup\left[\tilde{\theta}_{3}, \tilde{\theta}_{4}\right]$ with $\psi_{3}=0$. It is possible to switch to certain controls at points $\tilde{\theta}_{1} ; \tilde{\theta}_{2} ; \tilde{\theta}_{3} ; \tilde{\theta}_{4}$ as it is described in cases $7.3,7.3$, however, after passing two certain intervals with controls $(-1,-1),(1,-1) ;(-1,1),(1,1) ;(1,-1),(-1,-1) ;(1,1),(-1,1)$ correspondingly, we approach to the corresponding points $\tilde{\theta}_{4} ; \tilde{\theta}_{3} ; \tilde{\theta}_{2} ; \tilde{\theta}_{1}$, where we have can switch to uncertain control or continue with certain control according to the same pattern. 


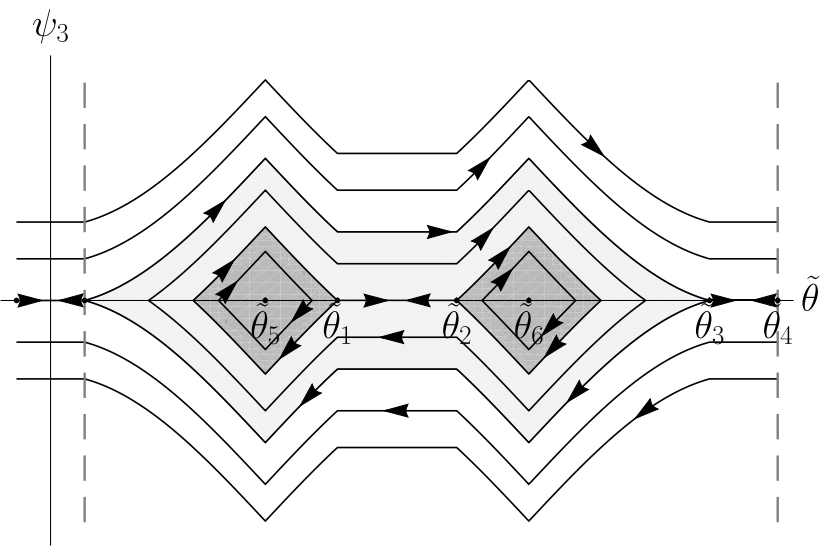

FigURE 62 . Phase portrait for $\Omega$-modifications of Reeds-Shepp problem in the cylinder $\left(\tilde{\theta}, \psi_{3}\right)$ for $\tilde{\Omega}$ presented in Figure 58.

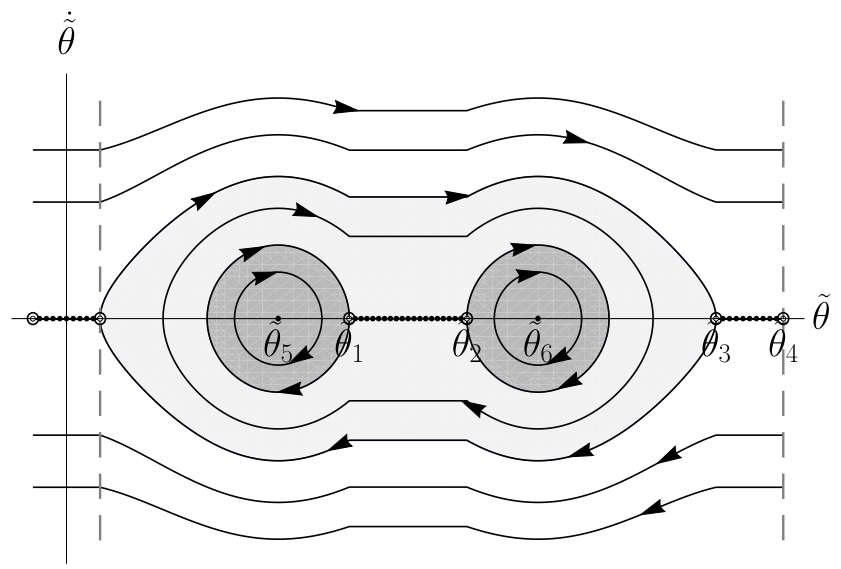

Figure 63. Phase portrait for $\Omega$-modifications of Sub-Riemannian problem on $\mathrm{SE}(2)$ in the cylinder $(\tilde{\theta}, \dot{\tilde{\theta}})$ for $\tilde{\Omega}$ presented in Figure 58 .

\subsection{Sub-Riemannian problem on $\mathrm{SE}(2)$}

An $\Omega$-modification of the sub-Riemannian problem on $\mathrm{SE}(2)$ is defined by (7.1)-(7.3) with (7.9).

The abnormal case $\psi_{0}=0$ is possible only as a trivial solution $u_{1}=u_{2}=0$.

Normal case: $\psi_{0}=-1$. From the maximality condition for $\mathcal{H}$ we have

$$
u_{1}=\psi_{1} \cos _{\Omega} \theta+\psi_{2} \sin _{\Omega} \theta, \quad \dot{\tilde{\theta}}=u_{2}=\psi_{3} .
$$

Suppose $\psi_{1}=\psi_{2}=0$, then $u_{1} \equiv 0$ and $\dot{\psi}_{3} \equiv 0$, therefore $u_{2} \equiv$ const, moreover we can assume w.l.o.g. $u_{2} \equiv \pm 1$. The corresponding trajectory is optimal up to infinity for "non-factorized problem".

If $\psi_{1}^{2}+\psi_{2}^{2} \neq 0$, then we assume $\psi_{1}^{2}+\psi_{2}^{2}=1$ w.l.g. Using the same notation as in (7.13) we get

$$
\dot{\psi}_{3}=\cos _{\tilde{\Omega}} \tilde{\theta} \sin _{\tilde{\Omega}^{\circ}} \tilde{\theta}^{\circ}
$$


with the maximized Hamiltonian $H=\frac{1}{2}\left(\cos _{\tilde{\Omega}}^{2} \tilde{\theta}+\dot{\tilde{\theta}}^{2}\right)$, which determines the phase portrait of system (7.17) (see Fig. 63). This Hamiltonian system (up to change $\tilde{\theta}$ for $\theta^{\circ}$ and $\tilde{\Omega}$ for $\Omega^{\circ}$ ) was considered in details in Section 5 and at the same time it is similar to the one considered in the previous Section 7.3, further we remark the main difference between them.

Since $\tilde{\Omega}$ is compact, we denote the minimal and maximal values of $\cos _{\tilde{\Omega}} \tilde{\theta}$ by $m_{1}$ and $m_{2}$ and assume $m_{1}^{2} \leq m_{2}^{2}$ without loss of generality. The following cases are possible for $H \geq 0$ :

- $H=0$, then we have only a trivial solution $u_{1}=u_{2}=0$.

- $H \in\left(0, m_{1}^{2}\right)$, using similar to case 7.3 (see Sect. 7.3) notation $\cos _{\tilde{\Omega}}^{-1} H=\left\{\tilde{\theta}_{+H}^{+}, \tilde{\theta}_{+H}^{-}\right\}, \cos _{\tilde{\Omega}}^{-1}(-H)=$ $\left\{\tilde{\theta}_{-H}^{+}, \tilde{\theta}_{-H}^{-}\right\}$we get inflectional solutions with small amplitudes for the angle parameter $\tilde{\theta} \in\left[\tilde{\theta}_{+H}^{+}, \tilde{\theta}_{-H}^{+}\right]$ and $\tilde{\theta} \in\left[\tilde{\theta}_{-H}^{-}, \tilde{\theta}_{+H}^{-}\right]$.

- $H=m_{1}^{2}$. If $\psi_{3}=0$, then we have fixed points for $\tilde{\theta} \in\left(\tilde{\theta}_{1}, \tilde{\theta}_{2}\right)$, points $\tilde{\theta} \in\left\{\tilde{\theta}_{1}, \tilde{\theta}_{2}\right\}$ are also fixed when $\Omega$ is $C^{2}$ in the neighborhood. If $\psi_{3} \neq 0$, then we have two small separatrices approaching to points $\tilde{\theta} \in\left\{\tilde{\theta}_{1}, \tilde{\theta}_{2}\right\}$ (for infinite time when $\Omega$ is $C^{2}$ in the neighborhood of the approaching points).

- $H \in\left(m_{1}^{2}, m_{2}^{2}\right)$, this case corresponds to inflectional solutions with big amplitude for angle $\tilde{\theta}$.

- $H=m_{2}^{2}$, similarly to case 7.4 we have fixed points at $\tilde{\theta} \in\left(\tilde{\theta}_{3}, \tilde{\theta}_{4}\right)$, two big separatrices for $\tilde{\theta} \in\left(\tilde{\theta}_{4}, \tilde{\theta}_{3}\right)($ $\bmod 2 \mathbb{S}$ ), which approach to points $\tilde{\theta} \in\left\{\tilde{\theta}_{3}, \tilde{\theta}_{4}\right\}$ for infinite time when $\Omega$ is $C^{2}$ in the neighborhood.

- $H \in\left(m_{2}^{2},+\infty\right)$; corresponding trajectories on $(x, y)$ are non-inflectional ones, since $\dot{\theta}=\psi_{3} \neq 0$.

Detailed analysis for cases $H=m_{1}^{2}$ and $H=m_{2}^{2}$ can be found in Section 4 (up to change $\tilde{\theta}$ to $\theta^{\circ}, H$ to $\mathbb{E}$ and $\cos _{\tilde{\Omega}}^{2} \tilde{\theta}$ to $\left.\mathcal{U}\left(\theta^{\circ}\right)\right)$.

\section{Plane DyNAmic MOtion}

In this section, we obtain exact formulae for extremals in the following problem with drift:

$$
T \rightarrow \min , \quad \ddot{x}=u \in \Omega,
$$

with some given initial conditions. Here $x=\left(x_{1}, x_{2}\right), u=\left(u_{1}, u_{2}\right)$, and $\Omega \subset \mathbb{R}^{2}$ is a compact convex set with $0 \in \operatorname{int} \Omega$. Put $\dot{x}=y$. The Pontryagin maximum principle gives $\mathcal{H}=p_{1} y_{1}+p_{2} y_{2}+q_{1} u_{1}+q_{2} u_{2}$, where $p$ and $q$ are conjugate variables to $x$ and $y$ correspondingly. Since $\dot{p}=-\mathcal{H}_{x}$ and $\dot{q}=-\mathcal{H}_{y}$, we have $p=p_{0}=$ const and $q=-p_{0} t+q_{0}$. Hence, for a.e. $t$, the control $u(t)$ moves along $\partial \Omega$ and the supporting half-plane to $\Omega$ at $u(t)$ is determined by $q(t)$. The main difficulty here is to describe this motion conveniently.

From the integrability point of view, we have a Hamiltonian system with 4 degrees of freedom, and it is easy to find 4 independent first integrals: $p_{1}, p_{2}, E=[p \times q]=p_{1} q_{2}-p_{2} q_{1}$, and the following nonsmooth Hamiltonian:

$$
H=\max _{u \in \Omega} \mathcal{H}=p_{1} y_{2}+p_{2} y_{2}+s_{\Omega}\left(q_{1}, q_{2}\right) .
$$

Obviously, the first integrals $p_{1}, p_{2}$, and $E$ are in involution. So, if $H$ is a smooth function outside $q_{1}=q_{2}=0$, then the system is integrable by Liouville-Arnold theorem. Unfortunately, $H$ is nonsmooth outside $q_{1}=q_{2}=0$ if $\Omega$ is not strongly convex. Moreover, to construct angle-momentum coordinates one should compute some explicit integration along basic loops on common level surfaces of the listed first integrals, which is not very simple procedure. Nonetheless, we are able to compute convenient formulae for extremals in terms of convex trigonometry. Put

$$
q_{1}=R \cos _{\Omega^{\circ}} \theta^{\circ} \quad \text { and } \quad q_{2}=R \sin _{\Omega^{\circ}} \theta^{\circ},
$$

where $R=s_{\Omega}\left(q_{1}, q_{2}\right)=H-p_{1} y_{1}-p_{2} y_{2} \geq 0$ is not constant along an extremal (in contrary to all previously considered problems). Nonetheless, the angle $\theta^{\circ}$ depends (locally) Lipschitz continuously on $t$ while $R \neq 0$. Using 
the generalized Pythagorean identity (see Sect. 2.2), we obtain $u_{1}=\cos _{\Omega} \theta$ and $u_{2}=\sin _{\Omega} \theta$ for some $\theta \leftrightarrow \theta^{\circ}$ if $R \neq 0$, since $q_{1} u_{1}+q_{2} u_{2}=R$. So we need to compute $\theta^{\circ}(t)$ to find the control $u$. Obviously,

$$
E=[p \times q]=R\left(p_{1} \sin _{\Omega^{\circ}} \theta^{\circ}-p_{2} \cos \Omega^{\circ} \theta^{\circ}\right) .
$$

We start with the simplest case $E=0$, i.e., when $p \| q$. If $p=0$, then $q=$ const and $\theta^{\circ}=$ const. Suppose $p \neq 0$. Then there exists a unique instant $t_{0}$, such that $q\left(t_{0}\right)=0$. Hence, $\theta^{\circ}(t)$ takes only two possible values one for $t<t_{0}$ and another for $t>t_{0}$. Both are solutions of the equation $p_{2} \cos \Omega^{\circ} \theta^{\circ}-p_{1} \sin _{\Omega^{\circ}} \theta^{\circ}=0$.

Now suppose that $E \neq 0$. In this case, let us express $R$ in $\theta^{\circ}$ by computing $\dot{\theta}^{\circ}$. The angle derivative formula in polar change of coordinates is the same in convex and classical trigonometries (see the inverse polar change of coordinates in Sect. 2.2). Hence, $\dot{\theta}^{\circ}=\left(q_{1} \dot{q}_{2}-\dot{q}_{1} q_{2}\right) / R^{2}=E / R^{2}$, and we obtain the following form of first integral $E$ for the Pontryagin system:

$$
E \dot{\theta}^{\circ}=\left(p_{2} \cos _{\Omega^{\circ}} \theta^{\circ}-p_{1} \sin _{\Omega^{\circ}} \theta^{\circ}\right)^{2} .
$$

Hence, if $\Omega^{\circ}$ has $C^{k}$ boundary, then $\theta^{\circ}(t) \in C^{k+1}$ by Proposition 2.5. Integrating equation (8.1), we obtain

$$
t / E=\int \frac{\mathrm{d} \theta^{\circ}}{\left(p_{2} \cos \Omega^{\circ} \theta^{\circ}-p_{1} \sin _{\Omega^{\circ}} \theta^{\circ}\right)^{2}} .
$$

Let us consider in detail two important shapes of $\Omega^{\circ}$. If $\Omega^{\circ}$ is an ellipse (see Sect. 2.3), then $\cos \Omega^{\circ} \theta^{\circ}=$ $a \cos s+x_{1}^{0}$ and $\sin _{\Omega^{\circ}} \theta^{\circ}=b \sin s+x_{2}^{0}$ where $\theta^{\circ \prime}{ }_{s}^{\prime}=a b+a x_{2}^{0} \sin s+b x_{1}^{0} \cos s$. Thus,

$$
t / E=\int \frac{a b+a y_{0} \sin s+b x_{0} \cos s}{\left(a p_{2} \cos s-b p_{1} \sin s+p_{2} x_{1}^{0}-p_{1} x_{2}^{0}\right)^{2}} \mathrm{~d} s .
$$

The last integral can be easily taken in elementary functions. Moreover,

$$
\begin{gathered}
u_{1}=\cos _{\Omega} \theta=\frac{d \sin _{\Omega^{\circ}} \theta^{\circ}}{\mathrm{d} \theta^{\circ}}=\frac{d\left(b \sin s+x_{2}^{0}\right)}{\mathrm{d} s} \frac{\mathrm{d} s}{\mathrm{~d} \theta^{\circ}}=\frac{b \cos s}{a b+a x_{2}^{0} \sin s+b x_{1}^{0} \cos s}, \\
u_{2}=\sin _{\Omega} \theta=\frac{-d \cos _{\Omega^{\circ}} \theta^{\circ}}{\mathrm{d} \theta^{\circ}}=\frac{d\left(-a \cos s-x_{1}^{0}\right)}{\mathrm{d} s} \frac{\mathrm{d} s}{\mathrm{~d} \theta^{\circ}}=\frac{a \sin s}{a b+a x_{2}^{0} \sin s+b x_{1}^{0} \cos s} .
\end{gathered}
$$

If $\Omega^{\circ}$ is a polygon, then $\cos _{\Omega^{\circ}} \theta^{\circ}$ and $\sin _{\Omega^{\circ}} \theta^{\circ}$ depend piecewise linearly on $\theta^{\circ}$, so equation (8.1) can be easily integrated in elementary functions as well.

\section{Conclusion}

Summarizing, we have derived formulae for extremals in a series of optimal control problems with twodimensional control lying in an arbitrary compact convex set $\Omega \subset \mathbb{R}^{2}$ with $0 \in \operatorname{int} \Omega$. Until now, formulae for extremals in these problems were known only for the case $\Omega$ being ellipse cetered at the origin (there is a small amount of exceptions mentioned in the Introduction). Investigation of optimal control problems involves a lot of work in addition to deriving formulae for extremals (such as verification of second order conditions, computing conjugate and cut times, finding closed trajectories and etc.). Nonetheless, precise investigations of optimal syntheses are almost always based on handy solutions to the Pontryagin maximum principle. Thus, we believe that the results obtained in the present paper open up a wide opportunity for precise investigations in considered optimal control problems, which were previously impossible.

Acknowledgements. The authors thank anonymous reviewers whose comments on presentation were taken into account. 


\section{REFERENCES}

[1] A.A. Agrachev, Rolling balls and octonions. Proc. Steklov Inst. Math. 258 (2007) 13-22.

[2] A. Agrachev and D. Barilari, Sub-Riemanian structures on 3D Lie groups. J. Dyn. Control Syst. 18 (2012) 21-44.

[3] A. Agrachev, D. Barilari and U. Boscain, A Comprehensive Introduction to Sub-Riemannian Geometry. Cambridge University Press (2019).

[4] A.A. Agrachev and Yu.L. Sachkov, Control Theory from the Geometric Viewpoint. Springer-Verlag, Berlin (2004).

[5] A.D. Alexandrov, Almost everywhere existence of the second differential of a convex function and some properties of convex surfaces connected with it, Leningrad State Univ. Annals [Uchenye Zapiski] Math. Ser. 6 (1939) 3-35.

[6] D.J. Balkcom and M.T. Mason, Extremal trajectories for bounded velocity mobile robots. Proceedings 2002 IEEE International Conference on Robotics and Automation (Cat. No.02CH37292) 2 (2002) 1747-1752.

[7] D.J. Balkcom and M.T. Mason, Time Optimal Trajectories for Bounded Velocity Differential Drive Vehicles. Int. J. Robotics Res. 21 (2002) 199-217.

[8] A. Bellaiche and J. Risler, Sub-Riemannian geometry. Progr. Math. 144 (1996).

[9] V.N. Berestovskii, Homogeneous manifolds with an intrinsic metric. II. Sib. Math. J. 30 (1989) $180-191$.

[10] V.N. Berestovskii, Geodesics of nonholonomic left-invariant intrinsic metrics on the Heisenberg group and isoperimetric curves on the Minkowski plane. Sib. Math. J. 35 (1994) 1-8.

[11] V.N. Berestovskii, I.A. Zubareva, Shapes of spheres of special nonholonomic left-invariant intrinsic metrics on some Lie groups. Sib. Math. J. 42 (2001) 613-628.

[12] I.Yu. Beschastnyi and Yu.L. Sachkov, Geodesics in the sub-Riemannian problem on the group SO(3). Sbornik Math. 207 (2016) 29-56.

[13] U. Boscain and F. Rossi, Invariant Carnot-Caratheodory metrics on $S^{3}, S O(3), S L(2)$ and Lens Spaces. SIAM J. Control Optim. 47 (2008) 1851-1878.

[14] U. Boscain, R. Duits, F. Rossi and Yu. Sachkov, Curve cuspless reconstruction via sub-Riemannian geometry. ESAIM: COCV 20 (2014) 748-770.

[15] U. Boscain, T. Chambrion and G. Charlot, Nonisotropic 3-level quantum systems: complete solutions for minimum time and minimum energy. Discrete Contin. Dyn. Syst. Ser. B 5 (2005) 957-990.

[16] E. Breuillard and E. Le Donne, On the rate of convergence to the asymptotic cone for nilpotent groups and subFinsler geometry. Proc. Natl. Acad. Sci. USA 110 (2013) 19220-19226.

[17] H. Busemann, The Isoperimetric Problem in the Minkowski Plane. Am. J. Math. 69 (1947) 863-871.

[18] Y.A. Butt, Yu.L. Sachkov and A.I. Bhatti, Extremal trajectories and Maxwell strata in sub-Riemannian problem on group of motions of pseudo-Euclidean plane. JDCS 20 (2014) 341-364.

[19] Y.A. Butt, Yu.L. Sachkov and A.I. Bhatti, Maxwell strata and conjugate points in sub-Riemannian problem on the Lie group SH(2). JDCS 22 (2016) 747-770.

[20] Y.A. Butt, Yu.L. Sachkov and A.I. Bhatti, Cut locus and optimal synthesis in sub-Riemannian problem on the lie group SH(2). JDCS 23 (2017) 155-195.

[21] H. Chitsaz, S.M. LaValle, D.J. Balkcom and M.T. Mason, Minimum wheel-rotation paths for differential-drive mobile robots. Proceedings 2006 IEEE International Conference on Robotics and Automation (2006) 1616-1623.

[22] R.C. Dalang, F. Dumas, S. Sardy, S. Morgenthaler and J. Vila, Stochastic optimization of sailing trajectories in an upwind regatta. J. Oper. Res. Soc. 66 (2015) 807-821.

[23] L.E. Dubins, On curves of minimal length with a constraint on average curvature, and with prescribed initial and terminal positions and tangents. Am. J. Math. 79 (1957) 497-516.

[24] L. Euler, Methodus inveniendi lineas curvas maximi minimive proprietate gaudentes, sive solutio problematis isoperimitrici latissimo sensu accepti. Lausanne: Bousquet (1744).

[25] D.S. Ferguson and P. Elinas, A Markov Decision Process Model for Strategic Decision Making in Sailboat Racing. Advances in Artificial Intelligence. Canadian AI. In vol. 6657 of Lecture Notes in Computer Science. Springer, Berlin, Heidelberg (2011) $110-121$.

[26] A.F. Filippov, Differential equations with discontunuous righthand sides. Kluwer (1988).

[27] I.A. Gribanova, The quasihyperbolic plane. Sib. Math. J. 40 (1999) 245-257.

[28] N. Jacobson, Lie Algebras. Interscience (1962).

[29] V. Jurdjevic, The geometry of the plate-ball problem. Arch. Rat. Mech. Anal. 124 (1993) 305-328.

[30] V. Jurdjevic and J. Zimmerman, Rolling Problems on Spaces of Constant Curvature. Lagrangian and Hamiltonian Methods for Nonlinear Control 2006, edited by F. Allgüwer et al. In Vol. 366 of Lecture Notes in Control and Information Sciences (2007).

[31] L.V. Lokutsievskiy, Convex trigonometry with applications to sub-Finsler geometry. Sb. Math. 210 (2019) $1179-1205$.

[32] L.V. Lokutsievskiy, Explicit formulae for geodesics in left invariant sub-Finsler problems on Heisenberg groups via convex trigonometry. To appear J. Dyn. Control Syst.

[33] A.A. Markov, Some examples of the solution of a special kind of problem on greatest and least quantities, Soobshch. Kharkovsk. Mat. Obshch. 1 (1887) 250-276 (in Russian).

[34] I. Moiseev and Yu.L. Sachkov, Maxwell strata in sub-Riemannian problem on the group of motions of a plane. ESAIM: COCV 16 (2010) 380-399.

[35] R. Montgomery, A tour of subriemannnian geometries, their geodesics and applications. Am. Math. Soc. (2002). 
[36] A. Philpott and A. Mason, Optimising Yacht Routes under Uncertainty. Proceedings of the 15th Chesapeake Sailing Yacht Symposium, Annapolis, Maryland, USA. P2001-5.

[37] L.S. Pontryagin, V.G. Boltyanskii, R.V. Gamkrelidze and E.F. Mishchenko, The mathematical theory of optimal processes. Wiley Interscience (1962).

[38] M. Rabaud, Optimal routing in sailing. Proceeding of the conference Sports Physics, June 8-10, Palaiseau, France (2016).

[39] J.A. Reeds and L.A. Shepp, Optimal paths for a car that goes both forwards and backwards. Pacific J. Math. 145 (1990) 367-393.

[40] R.T. Rockafellar, Convex Analysis. Princeton University Press (1997).

[41] Yu.L. Sachkov, Conjugate and cut time in sub-Riemannian problem on the group of motions of a plane. ESAIM: COCV 16 (2010) 1018-1039.

[42] Yu.L. Sachkov, Cut locus and optimal synthesis in the sub-Riemannian problem on the group of motions of a plane. ESAIM: COCV 17 (2011) 293-321.

[43] A. Sarti, G. Citti and J. Petitot, The symplectic structure of the primary visual cortex. Biol. Cybernet. 98 (2008) 33-48.

[44] D. Shelupsky, A generalization of the trigonometric functions. Am. Math. Monthly 66 (1959) 879-884.

[45] A.M. Vershik and V.Ya. Gershkovich, Nonholonomic dynamical systems. geometry of distributions and variational problems. Itogi Nauk. i Tekhn. Sovrem. Probl. Mat. Fund. Napravl. Dinam. Sistem. 7 (1986) 5-85 (In Russian).

[46] D. Wei, Y. Liu and M.B. Elgindi, Some generalized trigonometric sine functions and their applications. Appl. Math. Sci. 6 (2012) 6053-6068.

[47] R. Zarate-Minano, M. Anghel and F. Milano, Continuous wind speed models based on stochastic differential equations. Appl. Energy 104 (2013) 42-49. 